\title{
Computational transport, phase change and deposition analysis of inhaled multicomponent droplet-vapor mixtures in an idealized human upper lung model
}

\author{
Yu Feng ${ }^{\text {a }}$, Clement Kleinstreuer ${ }^{\text {a, b, * }}$, Nicolas Castro ${ }^{\mathrm{c}}$, and Ali Rostami ${ }^{\mathrm{c}}$ \\ ${ }^{a}$ Department of Mechanical and Aerospace Engineering, North Carolina State University, Raleigh, NC \\ 27695-7910, USA \\ ${ }^{\mathrm{b}}$ Joint UNC-NCSU Department of Biomedical Engineering, North Carolina State University, Raleigh, \\ NC 27695, USA \\ cAltria Client Services LLC., Research, Development and Engineering, 601 East Jackson Street, \\ Richmond, VA 23219, USA
}

\begin{abstract}
Numerous inhalable aerosols consist of multiple nano-to-microscale solid or liquid particles with dissolved or embedded compounds, as well as associated vapors. In general, of interest are the transport and conversion phenomena leading to local particle/droplet/vapor depositions. Selected examples include inhalation of aerosols from use of inhalers, cigarettes and electronic cigarettes. In this study the focus is on hygroscopic growth of nano-size multicomponent droplets and droplet-vapor interactions during transport with subsequent deposition in a human upper lung-airway model. For that purpose a comprehensive and efficient computational fluid-particle dynamics model has been developed. It is capable of simultaneously analyzing multi-component droplet-vapor and airflow interactions with evaporation and condensation effects for different sets of inhalation conditions. Selecting inhaled electronic cigarette (EC) aerosols as an application, the simulation results include detailed transport, deposition and absorption data for different constituents (i.e., water, propylene glycol, glycerol and nicotine) in both vapor and liquid forms for an idealized human upper lung airway geometry, i.e., from mouth to generation 3. Results indicate that liquid-vapor phase change induces hygroscopic growth of droplets, which in turn impacts significantly the deposition concentrations of aerosols via inertial impaction, secondary flows, Brownian motion, and the vapor-specific absorption rates. Parametric sensitivity analyses were performed to evaluate the influence of different inhalation flow waveforms on EC-aerosol transport, interaction, and deposition.
\end{abstract}

Keywords: Hygroscopic droplet growth; Multi-component droplet-vapor interaction modeling; Multi-component mixture plus discrete-droplet (MCM-DD) model; Inhaled droplet-reduction (IDR) method; Electronic cigarettes

${ }^{*}$ Corresponding author. Tel: +1-919-515-5216; fax: +1-919-515-7968

E-mail address: ck@ncsu.edu (C. Kleinstreuer) 


\section{Nomenclature}

\begin{tabular}{|c|c|}
\hline$A_{d}$ & Droplet surface area \\
\hline$c_{p}$ & Liquid specific heat \\
\hline$C_{C}$ & Cunningham correction factor \\
\hline$C_{m}, C_{T}$ & Knudsen number correction factors \\
\hline$\widetilde{D}_{a-v, s}$ & Diffusivity in the air \\
\hline$\widetilde{D}_{m, s}$ & Diffusivity in the mucus layer \\
\hline$d_{d}$ & Droplet diameter \\
\hline$d_{d, i n i}$ & Initial droplet diameter \\
\hline$d_{d, \max }$ & Maximum droplet hygroscopic diameter \\
\hline$d t_{d}$ & The droplet phase time differential \\
\hline$D E$ & Deposition efficiency \\
\hline$D E F$ & Deposition enhancement factor \\
\hline$D F$ & Deposition fraction \\
\hline$D P M$ & Discrete phase model \\
\hline$E C$ & Electronic cigarette \\
\hline$F_{i}^{D}$ & Drag force \\
\hline$F_{i}^{L}$ & Lift force \\
\hline$F_{i}^{B M}$ & Brownian motion-induced force \\
\hline$F_{i}^{G}$ & Gravity \\
\hline$G R$ & Growth Ratio \\
\hline$H$ & Helicity \\
\hline$H_{m}$ & The thickness of mucus layer \\
\hline$h_{\text {cell }}$ & The height of the near-wall prism mesh cell \\
\hline$j_{S}$ & Evaporation/condensation mass flux of the $\mathrm{s}^{\text {th }}$ component \\
\hline$k_{a-v}$ & Air-vapor mixture thermal conductivity \\
\hline$k_{d}$ & Droplet thermal conductivity \\
\hline$k_{H, s, a-m}$ & The equilibrium partition coefficient \\
\hline$K_{s}$ & Correction factor for Kelvin effect $K_{s}=\exp \left[4 \sigma_{s} M_{s} /\left(\mathfrak{R}_{f}\right.\right.$ \\
\hline
\end{tabular}




\begin{tabular}{|c|c|}
\hline Kn & Knudsen number $K n=2 \lambda_{a-v} / d_{d}$ \\
\hline$L$ & Latent heat of evaporation/condensation \\
\hline$m_{d}$ & Droplet mass \\
\hline$M M D$ & Mass median diameter \\
\hline$M_{S}$ & Molecular weight of species s \\
\hline$N_{d, \text { cell }}$ & The total droplet number in a specific mesh cell \\
\hline$N_{\text {dep }}$ & Number of deposited droplets \\
\hline$N u$ & Nusselt number $N u=\left(1+R e_{d} \operatorname{Pr}\right)^{1 / 3} \max \left[1, \operatorname{Re}_{d}^{0.077}\right]$ \\
\hline$R T$ & Respiratory tract \\
\hline $\operatorname{Pr}$ & Prandtl number $\operatorname{Pr}=c_{p} \mu / k_{a-\nu}$ \\
\hline$P_{v s, s a t}$ & Saturated vapor pressure \\
\hline$Q_{\text {in }}$ & Inhalation flow rate $[\mathrm{ml} / \mathrm{s}]$ \\
\hline$Q_{N, i n j}$ & Injected droplet number flow rate $[\# /(\mathrm{ml} \cdot \mathrm{s})]$ \\
\hline$Q_{N, \text { real }}$ & Realistic droplet number flow rate $[\# /(\mathrm{ml} \cdot \mathrm{s})]$ \\
\hline $\operatorname{Red}_{\mathrm{d}}$ & Droplet Reynolds number $\operatorname{Re}_{d}=\rho_{a-v}\left|\vec{v}_{a-v}-\vec{v}_{d}\right| d_{d} / \mu_{a-v}$ \\
\hline $\mathfrak{R}_{S}$ & Species gas constant \\
\hline$\Re$ & Universal gas constant \\
\hline$R D F$ & Regional deposition fraction \\
\hline RH & Relative humidity \\
\hline$S_{v-d}^{(E)}$ & $\begin{array}{l}\text { Latent heat of evaporation or condensation which is released or } \\
\text { absorbed by the droplets per local mesh cell }\end{array}$ \\
\hline$S c$ & Schmidt number $S c=v / D_{a-v}$ \\
\hline$S F$ & Scaling factor \\
\hline$S h$ & Sherwood number $S h=\left(1+R e_{d} S c\right)^{1 / 3} \max \left[1, R e_{d}^{0.077}\right]$ \\
\hline$t$ & Time \\
\hline$T$ & Temperature \\
\hline$T D F$ & Total deposition fraction \\
\hline$U$ & Continuous phase (air-vapor mixture) velocity \\
\hline$u d$ & Droplet velocity \\
\hline
\end{tabular}




\begin{tabular}{ll}
$\|\vec{V}\|$ & Fluid velocity magnitude \\
$X$ & Mole fraction \\
$Y$ & Mass fraction \\
Greek symbols & \\
$\alpha_{m}$ & Mass accommodation coefficient $\alpha_{m}=1$ \\
$\alpha_{T}$ & Thermal accommodation coefficient $\alpha_{T}=1$ \\
$\beta_{v-l, s}$ & Vapor-liquid mass change percentage of the s-th species \\
$\gamma_{S}$ & Activity coefficient of species s \\
$\Delta \mathrm{t}_{\mathrm{d}}$ & DPM time step \\
$\Delta \mathrm{t}_{\mathrm{f}}$ & Flow time step \\
$\lambda$ & Mean free path \\
$v$ & Kinetic molecular viscosity \\
$\rho_{a-v}$ & Air-vapor mixture density \\
$\rho_{d}$ & Droplet density \\
$\rho_{A}$ & Surface/Area density $\left[\mathrm{kg} / \mathrm{m}^{2}\right]$ \\
$\Gamma_{S, w}$ & Wall absorption coefficient for the s ${ }^{\text {th }} \mathrm{vapor}$ species \\
$\tau$ & Viscous shear stress \\
$\sigma$ & Surface tension \\
$\Phi$ & Dissipation function \\
$\mu$ & Dynamic viscosity \\
$\vec{v}$ & Fluid velocity \\
$\vec{\omega}$ & Fluid vorticity \\
\hline
\end{tabular}

\section{Subscripts}

A

$a b s$

air

$a-m$

$a-v$

cond
Area

Absolute

Dry air

Air-mucus layer

Air-vapor mixture (continuous phase)

Condensation 


\begin{tabular}{|c|c|}
\hline$d$ & Droplet or liquid phase \\
\hline env & Environmental or ambient \\
\hline$e q$ & Equilibrium \\
\hline $\mathrm{H}_{2} \mathrm{O}$ & Water \\
\hline$i$ & Tensor index \\
\hline$j$ & Tensor index \\
\hline$k$ & Droplet index \\
\hline$l$ & Liquid form \\
\hline$v-l$ & Vapor-liquid \\
\hline$m$ & Mass \\
\hline$s$ & Component index \\
\hline$T$ & Thermal \\
\hline tot & Total \\
\hline$v$ & Vapor phase or vapor form \\
\hline$v-d$ & Vapor-droplet \\
\hline vsat & Saturated vapor \\
\hline vsurf & Vapor at the droplet surface \\
\hline$w$ & Wall \\
\hline \multicolumn{2}{|c|}{ Superscripts } \\
\hline dep & Deposition \\
\hline E & Energy \\
\hline in & Inflow \\
\hline$i n i$ & Initial \\
\hline out & Outflow \\
\hline sus & Suspended \\
\hline
\end{tabular}




\section{Introduction}

Inhalable liquid aerosols generated from selected sources such as inhalers, drug delivery devices, cigarettes and electronic cigarettes involve complex physical and thermodynamic interactions between, air, vapor, particles and respiratory tract walls. Electronic cigarette (EC) aerosols typically contain interacting droplet-vapor mixtures with nicotine, glycerol, propylene glycol (PG) and added flavors. Understanding the vapor particle interactions and dynamics of aerosol transport and deposition is important because these micro-scale phenomena ultimately determine the fate of the inhaled aerosols.

In contrast to experimental and clinical studies, numerical methods, i.e., computational fluid-particle dynamics (CF-PD) simulations, provide a non-invasive, and relatively inexpensive way to predict local deposition and absorption data for different constituents in both vapor and liquid forms. Results from validated computer simulation models can inform and strengthen the scientific understanding of lung deposition patterns expected from various electronic cigarettes.

As recently reviewed by Tian et al. (2015), there are different types of numerical models for exposure studies of which semi-empirical and 1-D whole-lung models are most frequently used. In contrast to these two approaches, CF-PD models are based on first principles of physics with a minimum number of empirical correlations. Papers discussing the fate of inhaled EC aerosols include Manigrasso et al. (2015 a \& b) who computed the EC-particle deposition fractions in a stochastic lung model using the Multiple-Path Particle Dosimetry approach. However, by neglecting droplet-vapor phase change during transport, they were not able to provide deposition data for the accumulated mass dose of different constituents after EC-aerosol inhalation. Recently, using a CF-PD model, Schoroeter et al. (2016) investigated size-change dynamics of single-component droplets with different initial diameters in nasal passages under stead-state inspiratory conditions. The model can simulate vapor transport; however, without the vapor-concentration changes induced by the evaporation/condensation effects. Several papers (Vansickel et. al., 2014, Lee et al., 2015; Behar et al., 2015; Farsalinos et al., 2013; among others) investigated puffing behavior and nicotine uptake when using ECs. Behar et al. (2015) investigated the intake of different EC products and claimed that realistic inhalation flow rates range from $12 \mathrm{ml} / \mathrm{s}$ to $26 \mathrm{ml} / \mathrm{s}$ and the puff duration is from 1.67 seconds to $3.63 \mathrm{~seconds}$. Lee et al. (2015) also measured the puff volume and flow rate of different smokers. Evan and Hoffman (2014) reviewed contributions before 2014 on puffing topography when using EC. Generally, 
cigarette smoker who use E-cigarette take shorter puffs at higher puff rate, while experienced Ecigarette users take longer puffs at lower air volume rate (Vansickel et.al. 2014). Longer puffs for EC consumption were reported when compared to TC consumptions. It has been reported that the intake concentrations of vapor species and droplet number will not be influenced by the inhalation flow rate for EC smoking which is not true for conventional cigarette smoking (see Talih et al., 2015). However, at high airflow rates, the excessive cooling of the heating element results in reduction of aerosol delivery and particle numbers. Also of great interest is the paper by Alderman et al. (2014) on measured glycerin, PG and nicotine, generated by several EC products in droplets and vapors.

The major challenges in detailed modeling of the fate of inhaled EC-aerosols arise from the complexities of the very high numbers of polydisperse multi-component droplets, their varying droplet-vapor-air inlet conditions with subsequent size-change dynamics and vapor interactions, large system-scale variations, as well as the non-uniform geometries of any given representative or subject-specific respiratory system. For example, existing CF-PD models neglect the interactions between droplets and vapors during their transport; however, those mechanisms are essential for an improved understanding and a better prediction of local aerosol transport and deposition. We developed a comprehensive Multi-Component Mixture plus Discrete Droplet (MCM-DD) model, which is capable of simultaneously analyzing multicomponent droplet-vapor airflow interaction dynamics with evaporation and condensation effects for any set of inhalation conditions. Specifically, the major mechanisms covered by the MCM-DD model are: direct vapor species deposition, vapor species deposition from evaporation, direct liquid species deposition in droplets, and liquid species deposition by condensation. Not being limited to cigarette smoke, the general MCM-DD model is capable of simulating the transport and deposition of other inhalable droplet-vapor aerosol mixtures as well.

The present study provides results on the local deposition of different species in the multicomponent droplet-vapor aerosol mixture when using ECs. Specifically, the significance of droplet-vapor interaction with phase-change, the impact of different puff topographies (i.e., steady-state and transient waveforms with different holding durations), parametric sensitivity analyses on EC aerosol transport and deposition in the idealized human upper lung airway geometry (from mouth to G3) are discussed. The impact of E-liquid composition which affects the liquid vapor partitioning, such as the activity coefficient can also be taken into account. In 
addition, because of the sheer number of inhaled particles, the inhaled droplet-reduction (IDR) method is introduced to gain realistic simulation results for liquid-vapor interactions at reasonable computational cost.

\section{Theory}

To encompass the major mechanisms based on first principles, we developed a comprehensive, accurate and efficient CF-PD solver, which entails the MCM-DD and IDR models. It is capable of simultaneously simulating, for any set of puffing/inhalation conditions, multicomponent droplet-vapor airflow dynamics with evaporation and condensation effects during their transport in human respiratory systems. The governing equations are outlined next.

\subsection{Continuous Phase (Air-Vapor Mixture) Transport}

Any given air-vapor mixture is described as a single continuous phase, i.e., the conservation laws for mass, momentum and energy describe the air-vapor mixture transport and the advection-diffusion equation describes vapor-species transport. Droplet-vapor interaction, i.e., liquid-vapor mass change due to evaporation or condensation, are implemented by: (i) introducing source terms into the energy equation and vapor-species transport equations (see Eqs. (1) to (4)); and (ii) employing the local vapor-mass fraction in the droplet mass conservation equation (see Eq. (5)). Specifically, the energy equation of the air-vapor mixture reads:

$$
\begin{aligned}
& \frac{\partial\left(\rho c_{p} T\right)_{a-v}}{\partial t}+\frac{\partial\left(\rho c_{p} u_{j} T\right)_{a-v}}{\partial x_{j}}= \\
& \frac{\partial}{\partial x_{j}}\left[\left(k_{a-v}+\frac{\rho_{a-v} c_{a-v, p} v_{T}}{\operatorname{Pr}_{T}}\right) \frac{\partial T_{a-v}}{\partial x_{j}}\right]+\Phi+\frac{\partial}{\partial x_{j}}\left[\sum_{s=1}^{N} h_{s} \rho_{a-v}\left(\tilde{D}_{a-v}+\frac{v_{T}}{S c_{T}}\right) \frac{\partial Y_{s}}{\partial x_{j}}\right]+S_{v-d}^{(E)}
\end{aligned}
$$

In Eq. (1), the energy source term $S_{v-d}^{(E)}$ is the latent heat of evaporation or condensation which is released or absorbed by the droplets per local mesh cell:

$$
S_{v-d}^{(E)}=\sum_{s=1}^{4} S_{v-d, s}^{(E)}=\left\{\sum_{i=1}^{N_{d, c e l l}}\left[\left(\sum_{s=1}^{4} L_{s} \overline{j_{s}}\right) A_{d}\right]_{i}\right\} / V_{\text {cell }}
$$

where $N_{d, \text { cell }}$ is the total droplet number in a specified mesh cell.

The governing equation for advection and diffusion of the $s^{\text {th }}$ vapor species are expressed as: 


$$
\frac{\partial\left(\rho_{a-v} Y_{s, v}\right)}{\partial t}+\frac{\partial}{\partial x_{j}}\left(\rho_{a-v} u_{j} Y_{s, v}\right)=\frac{\partial}{\partial x_{j}}\left[\rho_{a-v}\left(\tilde{D}_{a-v, s}+\frac{v_{T}}{S c_{T}}\right) \frac{\partial Y_{s, v}}{\partial x_{j}}\right]+S_{v-d, s}^{(Y)}
$$

where $S c_{T}=0.9$ is the turbulence Schmidt number, $v_{T}$ is the turbulent viscosity, and $\widetilde{D}_{a-v, S}$ is the molecular diffusivity of the $\mathrm{s}^{\text {th }}$ vapor species in the air-vapor mixture. The local vaporized/condensed vapor-mass flow rate of the aerosol components are added to its advectiondiffusion equation as a source term $S_{v-d, s}^{(Y)}\left(\mathrm{kg} \mathrm{m}^{-3} \mathrm{~s}^{-1}\right)$, i.e.,

$$
S_{v-d, s}^{(Y)}=\int_{t_{i, \text { start }}}^{t_{i, \text { start }}+\Delta t_{f}}\left(\sum_{i=1}^{N_{d, c e l l}}\left(\overline{j_{s}} A_{d}\right)_{i}\right) d t_{d} /\left(V_{\text {cell }} \Delta t_{f}\right)
$$

where $\overline{j_{S}}$ is the average evaporation/condensation mass flux normal to the droplet surface of the $\mathrm{s}^{\text {th }}$ component (i.e., $\overline{j_{s}}>0$ for evaporation and $\overline{j_{s}}<0$ for condensation); and $A_{d}$ is the droplet surface area. Also, $\mathrm{dt}_{\mathrm{d}}$ represents the droplet phase time differential and $\Delta \mathrm{t}_{\mathrm{f}}$ is the flow time-step.

\subsection{Multicomponent Droplet Size-Change and Transport}

The Lagrangian approach is chosen to track multi-component droplets, neglecting rotational motion, if any. The governing equations for discrete droplets are the translational equation of droplets as well as the mass and energy conservation laws for droplets.

\subsubsection{Translational Equation of Droplets}

Newton's second law of motion states:

$$
\frac{d}{d t}\left(m_{d} u_{d, i}\right)=F_{i}^{D}+F_{i}^{L}+F_{i}^{B M}+F_{i}^{G}
$$

where $F_{i}^{D}, F_{i}^{L}, F_{i}^{B M}$, and $F_{i}^{G}$ are the drag force, lift force, Brownian motion-induced force, and gravity, respectively.

\subsubsection{Mass Conservation of Droplets}

Droplet mass changes due to condensation/evaporation can be described as:

$$
\frac{d m_{d}}{d t}=-\sum_{s=1}^{m} \int_{\text {surf }} j_{s} d A \approx-\sum_{s=1}^{m}\left(\overline{j_{s}} \cdot A_{d}\right)
$$

where the average mass flux $\overline{j_{s}}$ is given by Turns (1996):

$$
\overline{j_{s}}=\rho_{a-v} \cdot C_{m} \cdot S h \cdot \tilde{D}_{a-v, s} \cdot d_{d}^{-1} \ln \left[\left(1-Y_{\mathrm{s}, \mathrm{v}, \text { cell }}\right) /\left(1-Y_{s, \text { surf }}\right)\right]
$$

Here, Sh is the Sherwood number (see the Nomenclature for definition), while $Y_{s, v s u r f}$ and $Y_{s, v, c e l l}$ are the mass fractions of the $s^{\text {th }}$ vapor phase at the droplet surface and at the center of the 
cell where the droplet currently resides. The correction factor $C_{m}$ for submicron droplets, considering non-continuum effects, can be expressed as:

$$
C_{m}=\frac{1+K n}{1+\left(\frac{4}{3 \alpha_{m}}+0.377\right) K n+\frac{4}{3 \alpha_{m}} K n^{2}}
$$

where, $\mathrm{Kn}$ is the Knudsen number, $K n=2 \lambda_{a-v} / d_{d}$, in which $\lambda_{a-v}$ is the mean free path of the air-vapor mixture surrounding the droplet; and $\alpha_{m}$ is the mass accommodation coefficient, where $\alpha_{m}=1$ was used in the present study (Hinds, 1999). The derivation of Eq. (7) is based on the assumption that the distance between the droplet mass center and the mesh cell center is much larger than the droplet radius. Specifically, $Y_{s, v, c e l l}$ is determined by the advectiondiffusion equation (see Eq. (3)), while $Y_{s, v s u r f}$ can be calculated by:

$$
Y_{s, v s u r f}=\frac{\rho_{s, v s u r f}}{\rho_{a-v}}=\frac{\left(P_{s, v s u r f} / \Re_{s} T_{d}\right)}{\rho_{a-v}}=\frac{\gamma_{s} \cdot K_{s} \cdot X_{s, d} \cdot P_{s, v s a t}\left(T_{d}\right)}{\rho_{a-v}\left(\Re / M_{s}\right) T_{d}}
$$

Here, $\rho_{s, v s u r f}$ is the equivalent density of vapor species $s$ in the air-vapor mixture at the droplet surface; $\gamma_{s}$ is the activity coefficient of species $\mathrm{s}$, which is a correction of the evaporation/condensation characteristics of a certain liquid component due to the molecular bonding changes in the pure droplets and in the multicomponent droplets ( $\mathrm{Tu}$ and Ray, 2005) ; $X_{s, d}$ is the mole fraction of the $\mathrm{s}^{\text {th }}$ component in the droplet; $\mathfrak{R}_{s}$ is the species gas constant; $T_{d}$ is the droplet temperature; and $P_{s, v s a t}$ is the temperature-dependent saturation pressure of the pure $\mathrm{s}^{\text {th }}$ species. Clearly, parameters $\gamma_{s}$ and $X_{s, d}$ serve as corrections to the vapor pressure at the surface of multicomponent droplets (Raoult's law), while $K_{S}$ is the correction factor for the Kelvin effect (Hinds, 1999):

$$
K_{s}=\exp \left[4 \sigma_{s} M_{s} /\left(\Re \rho_{s} d_{d} T_{d}\right)\right]
$$

where $\sigma_{S}$ is the surface tension of component s at the droplet surface.

\subsubsection{Energy Conservation of Droplets}

The droplet heat transfer equation takes on the form:

$$
m_{d} c_{p, d}\left(d T_{d} / d t\right)=C_{T}\left(k_{a-v} \cdot N u / d_{d}\right)\left(T_{a-v, \text { cell }}-T_{d}\right) A_{d}-\left(\sum_{s=1}^{N} L_{s} \overline{j_{s}}\right) A_{d}
$$


where $L_{s}$ is the latent heat of liquid-vapor phase transition of the $\mathrm{s}^{\text {th }}$ species $\left(L_{s}\right.$ is always larger than zero). Also, $C_{T}$ is the correction factor for submicron droplets:

$$
C_{T}=\frac{1+K n}{1+\left(\frac{4}{3 \alpha_{T}}+0.377\right) K n+\frac{4}{3 \alpha_{T}} K n^{2}}
$$

where $\alpha_{T}$ is the thermal accommodation coefficient with the value of $\alpha_{T}=1$ (Hinds, 1999).

\subsection{Boundary Conditions}

For realistic numerical simulations of EC-aerosol transport and deposition in human respiratory systems, initial and boundary conditions for different variables were selected in plausible ranges from the open literature.

\subsubsection{Mouth-inlet Conditions}

Considering first steady inlet conditions, an average inlet velocity was determined from a representative inhalation flow rate at mouth-inlet, i.e., $Q_{\text {in }}=27.5 \mathrm{ml} / \mathrm{s}$ (Canada, 2014). The average inlet velocity was $0.5459 \mathrm{~m} / \mathrm{s}$, while the inlet aerosol temperature was $360.15 \mathrm{~K}$. That flow rate was also chosen for the mesh-independence test. Then, for parametric sensitivity analyses, different steady-state inhalation flow rates ( $Q_{\text {in }}=12 \mathrm{ml} / \mathrm{s}, 20 \mathrm{ml} / \mathrm{s}$, and $27.5 \mathrm{ml} / \mathrm{s}$ ), puffing durations ( 2 seconds and 3 seconds), and holding durations ( 0 second and 1 second) were selected, representing different EC-puffing/inhalation behaviors (Behar et al., 2015; Lee et al., 2015; among others). For example, Waveform \#1 is typical for conventional cigarette smokers switching to ECs, with a puffing duration of 2.4 seconds and a total volume of $63.9 \mathrm{ml}$ (see Fig. 1 (a)). Waveform \#2 (see Fig. 1 (b)) was based on regular EC-users, where the puffing duration is 5.0 seconds but the total volume remains at $63.9 \mathrm{ml}$.

To simulate continuous inhalation of EC droplets, multiple droplet injections were generated based on random-parabolic droplet distributions at the mouth inlet, using an in-house MatLab $^{\circledR}$ code (Feng and Kleinstreuer 2014). The droplet initial diameter was uniformly $400 \mathrm{~nm}$, which represents the MMD of a realistic EC droplet size distribution (Alderman et al., 2014). Liquid-vapor partitions and other initial inputs (see Table 1) were in reasonable ranges, considering the most recent EC aerosol measurements (Alderman et al., 2014; among others). 


\subsubsection{Boundary Conditions for Multicomponent Droplet and Vapor Depositions}

To evaluate potential uptake of certain components in EC aerosols, accurate calculations of the locally deposited/absorbed amounts are of great importance; hence, realistic wall boundary conditions for droplet and vapor deposition/absorption are necessary.

\section{Droplet deposition boundary condition:}

Droplet deposition occurs when its mass center comes within the droplet radius from the wall. The species mass deposited in the droplet with index $\mathrm{k}$ is calculated as:

$$
\left[m_{s, l}^{(d e p)}\right]_{k}=\left[m_{d} Y_{s, d}\right]_{k}
$$

where $\mathrm{s}$ is the species index, and $Y_{s, d}$ is the mass fraction of the s-th species in the deposited droplet.

\section{Vapor deposition boundary condition:}

Vapor absorption at human lung-airway surfaces is a function of tissue solubility, which is related to the air-lung partitioning ratio. In the present study, quasi-steady equilibrium at the air-mucus interface (see Fig. 2 (a)) and zero concentration below the lipid layer due to the rapid removal of vapor molecules by the tissue were assumed. Specifically, "quasi-steady equilibrium" means that the species concentrations at both sides of the interface always satisfy Henry's law. Thus, the vapor absorption boundary conditions at lung-airway walls $(n=0)$ for glycerol, PG and nicotine are:

$$
\left.\frac{\partial Y_{s, v}}{\partial n}\right|_{n=0}+\left.\Gamma_{s, w} \cdot Y_{s, v}\right|_{n=0}=0 \quad\left(\left.Y_{s, v}\right|_{n=0} \geq 0\right)
$$

Here, $\Gamma_{s, w}$ is called the wall absorption coefficient for the $\mathrm{s}^{\text {th }}$ vapor species, which is:

$$
\Gamma_{s, w}=\frac{\tilde{D}_{m, s}}{\tilde{D}_{a-v, s} k_{H, s, a-m} H_{m}}
$$

where $\widetilde{D}_{a-v, s}$ and $\widetilde{D}_{m, s}$ are the vapor diffusivity in the air and the mucus phase, respectively; $H_{m}$ is the thickness of the mucus layer which is assumed to be $10 \mu \mathrm{m}$ (Zhang et al., $2012 \mathrm{~b}$ ); and $k_{H, s, a-m}$ is the dimensionless gas-liquid equilibrium partition coefficient (i.e., dimensionless Henry's law constant) for a given vapor species (Pankow, 2001). Specifically, being a third-type boundary condition, Eq. (14) is more realistic than a first- or second-type because it represents the finite absorption rate of vapor species (Keyhani et al., 1997). In contrast, the widely used 
zero vapor-concentration, i.e., the first-type boundary condition, indicates an infinite absorption rate. Actually, the mucus-layer thickness for healthy human $H_{m}$ increases from the distal to the proximal lung airways by $50 \mu \mathrm{m}$ (Fahy and Dickey, 2010), where the average mucus-layer thickness is $15 \mu \mathrm{m}$ in the mouth-throat region, $11 \mu \mathrm{m}$ in G1-G3, and $6 \mu \mathrm{m}$ in G4-G9 (ICRP, 1994; Luchtel, 1978). This justifies an average value of $\mathrm{H}_{\mathrm{m}}=10 \mu \mathrm{m}$.

The calculation procedure for the wall absorption rates of vapor species is based on the numerical treatment documented in Shi et al. (2004). Assuming that the mass fraction distribution is a linear function of the distance from the near-wall center to the wall (Fig. 2 (b)), the wall mass fractions are calculated and updated at each time step according to the mixed boundary condition:

$$
\left.\frac{\partial Y_{s, v}}{\partial n}\right|_{w}=\frac{\left.Y_{s, v}\right|_{c}-\left.Y_{s, v}\right|_{w}}{0.5 h_{\text {cell }}}=\left.\Gamma_{s, w} \cdot Y_{s, v}\right|_{w}
$$

where $\left.Y_{s, v}\right|_{c}$ is the mass fraction at the near-wall mesh cell center, and $\mathrm{h}_{\text {cell }}$ is the height of the near-wall prism mesh cell. Therefore, $\left.Y_{s, v}\right|_{w}$ can be calculated as:

$$
\left.Y_{s, v}\right|_{w}=\left.\left(1+0.5 h_{\text {cell }} \Gamma_{s, w}\right)^{-1} Y_{s, v}\right|_{c}
$$

$\left.Y_{s, v}\right|_{w}$ in Eq. (16) is identical to $\left.Y_{s, v}\right|_{n=0}$ in Eq. (14). After the droplet touches the wall, it is assumed that all the components are rapidly absorbed and will not be able to re-evaporate into the lung airways which behave only as sinks. Thus, the local vapor-species absorption flux by diffusion, without turbulence effects, near the lung airway walls (in $\left[\mathrm{kg} /\left(\mathrm{m}^{2} \mathrm{~s}\right)\right]$ ) reads:

$$
\left.j_{s, v}^{(d e p)}\right|_{w}=-\left.\rho_{a-v} \tilde{D}_{a-v, s} \frac{\partial Y_{s, v}}{\partial n}\right|_{w}=\left.\rho_{a-v} \tilde{D}_{a-v, s} \Gamma_{s, w} \cdot Y_{s, v}\right|_{w}
$$

Accordingly, the total mass deposition of one species (e.g., glycerol, PG, or nicotine) can be expressed as the summation of the mass deposition of the species in droplet and vapor forms. Clearly, even under steady inhalation conditions, the total mass deposition is a function of both space and time. The total deposited mass of the $\mathrm{s}^{\text {th }} \mathrm{EC}$-aerosol species is then:

$$
m_{s}^{(d e p)}(t)=m_{s, l}^{(d e p)}(t)+m_{s, v}^{(d e p)}(t)
$$


where $m_{s, l}^{(d e p)}(t)$ and $m_{s, v}^{(d e p)}(t)$ is the accumulated deposited mass [kg] of the $\mathrm{s}^{\text {th }}$ species in liquid and vapor forms, respectively, where $m_{s, l}^{(d e p)}(t)$ and $m_{s, v}^{(d e p)}(t)$ are evaluated using the following equations:

$$
m_{s, v}^{(d e p)}(t)=\left.\iint j_{s, v}^{(d e p)}\right|_{w} d t d A
$$

and

$$
m_{s, l}^{(d e p)}(t)=\int \sum_{N_{d e p}}\left[m_{s, l}^{(d e p)}\right]_{k} d t
$$

Here, $\left.j_{s, v}^{(d e p)}\right|_{w}$ is obtained with Eqs. (18 a and $\left.18 \mathrm{~b}\right)$ and $\left[m_{s, l}^{(d e p)}\right]_{k}$ by Eq. (13).

\subsubsection{Other Wall Boundary Conditions}

A constant wall temperature of $37^{\circ} \mathrm{C}$ (i.e., $310.15 \mathrm{~K}$ ) and constant relative humidity (RH) of $99.5 \%$ were assumed at the walls of the human respiratory system and uniform (zero) pressure was applied at the eight outlets (see Feng et al. 2015).

\subsection{Deposition calculations of components in EC aerosols}

Droplet-vapor mixture deposition in human respiratory systems are traditionally quantified in terms of the deposition efficiency (DE), the deposition fraction (DF), or the deposition enhancement factor (DEF) (see Zhang et al., 2012 a \& b). However, those variables are not sufficient to provide complete physical insight for aerosol deposition in dual (i.e., liquid and vapor) phases as a result of evaporation and condensation. Thus, to provide more relevant deposition data for different species in the inhaled aerosol mixture, definitions of modified deposition fractions are introduced. Specifically, to measure the deposition fractions of each species in liquid or vapor form, the deposition fraction based on total species inflow mass are now defined as:

$$
D F_{s, \frac{l}{v+l}}(t)=\frac{m_{s, l}^{(d e p)}(t)}{m_{s, l}^{(i n)}(t)+m_{s, v}^{(i n)}(t)}
$$

and

$$
D F_{s, \frac{v}{v+l}}(t)=\frac{m_{s, v}^{(d e p)}(t)}{m_{s, l}^{(i n)}(t)+m_{s, v}^{(i n)}(t)}
$$

where $D F_{s, \frac{l}{v+l}}$ and $D F_{s, \frac{v}{v+l}}$ are the deposition fractions of s-th species in liquid and vapor forms,

respectively; where $m_{s, l}^{(i n)}(t)$ and $m_{s, v}^{(i n)}(t)$ are the total inflow mass of the s-th species in liquid 
and vapor forms. Hence, the deposition fraction of the s-th species $D F_{S}(t)$ is the summation of $D F_{s, \frac{l}{v+l}}$ and $D F_{s, \frac{v}{v+l}}$. However, because of the strong variations in liquid-vapor partitions and mass exchanges, it is also necessary to introduce a vapor deposition fraction based on vapor species inflow mass $D F_{s, \frac{v}{v}}(t)$ as well as a liquid deposition fraction based on liquid species inflow mass $D F_{s, \bar{l}} l(t)$ :

$$
D F_{s, \frac{v}{v}}(t)=\frac{m_{s, v}^{(d e p)}(t)}{m_{s, v}^{(i n)}(t)}
$$

and

$$
D F_{s, \frac{l}{l}}(t)=\frac{m_{s, l}^{(d e p)}(t)}{m_{s, l}^{(i n)}(t)}
$$

\subsection{Inhaled Droplet-Reduction (IDR) Method}

For realistic simulations of EC droplet-vapor interactions the very high number density of injected droplets (i.e., $5.5 \mathrm{e}+10 / \mathrm{cc}$ ) has to be considered, which implies considerable computational cost when using the Lagrange tracking scheme. In order to reduce the computational time, a minimal, scaled-down droplet-number flow rate $Q_{N, i n j}[\# /(\mathrm{ml} s)]$ has to be considered, representing the impact of the real droplet-number flow rate $Q_{N}$, real $[\# /(\mathrm{ml} s)]$. This task has been accomplished with the "inhaled droplet-reduction" (IDR) method which must satisfy two requirements:

- The assumed droplet-number flow rate, $Q_{N, i n j}$, must be sufficiently high to guarantee particle deposition patterns basically independent of the actual droplet-injection number, $Q_{N, \text { real. }}$

- Nevertheless, when using a lower number of inhaled droplets to represent the actual number of droplets generated from the EC, the droplet-vapor interaction rate (i.e., liquid-vapor mass transfer rate) for a single droplet must be modified by a scaling factor to maintain the accuracy of interaction mechanisms between the two phases.

- Expanding from a single droplet to $\mathrm{Q}_{\mathrm{N}, \mathrm{rea}} / \mathrm{Q}_{\mathrm{N}, \mathrm{inj}}$ droplets, the original droplet-vapor source term has to be amplified by the $\mathrm{Q}_{\mathrm{N}, \mathrm{real}} / \mathrm{Q}_{\mathrm{N}, \mathrm{inj}}$ ratio.

Indeed, it has been observed from our EC droplet-vapor aerosol transport/deposition simulations that: (a) the total deposition efficiency of the key constituent, i.e., nicotine, converges as the injected droplet number increases; and (b) the single droplet-vapor interaction 
intensity varies with the increase in droplet number. Based on these facts, the goal is to reduce the computational cost induced by the realistic high droplet-injection number flow rate by correcting droplet-vapor interaction intensities with a scaling factor (SF) which is the kernel of the IDR method. For a single droplet, the liquid-vapor interaction intensity at time $t$ is defined as the mass change percentage due to the condensation or evaporation, where the scaling factor (SF) is the non-dimensionalized single-droplet liquid-vapor interaction intensity adjustment, with the condition that $\mathrm{SF}=1$ when $\mathrm{Q}_{\mathrm{N}, \text { real }}=\mathrm{Q}_{\mathrm{N}, \mathrm{inj}}$. Based on computational experiments, an SF-correlation has been determined for a manageable number-density of injected nano-droplets, i.e., presently $\mathrm{Q}_{\mathrm{N}, \mathrm{inj}}=207[\# /(\mathrm{ml} \mathrm{s})]$. This allows to simulate efficiently the droplet-vapor interaction intensity (i.e., correct size-change dynamics) for the actual droplet-number flow rate, QN,real.. In summary, the transport and deposition of $\mathrm{Q}_{\mathrm{N}, \mathrm{inj}}=207[\# /(\mathrm{ml} \mathrm{s})]$ with corrected size-change dynamics due to evaporation/condensation can statistically represent the real droplet-number flow rate $\mathrm{Q}_{\mathrm{N}, \text { real. }}$

The SF-correlation, being a function of $\mathrm{Q}_{\mathrm{N}, \text { real./ }} \mathrm{Q}_{\mathrm{N}, \mathrm{inj}}$, has been obtained from the single droplet-vapor interaction intensity data shown in Fig. 3:

$$
S F=\left(Q_{N, \text { real }} / Q_{N, i n j}\right)^{-0.4643}
$$

It is assumed that (1) this relationship is equally valid for other constituents in the aerosol and (2) it is geometry independent. The SF-values for different inhalation conditions used in the present study are listed in Table 3. Assuming that the SF-correlation will not be influenced by variations of droplet composition and size, the average evaporation/condensation mass flux $\overline{j_{s}}$ (see Eq. (7)) can be modified as:

$$
\overline{j_{s}}=S F \cdot \rho_{a-v} \cdot C_{m} \cdot S h \cdot \tilde{D}_{a-v, s} \cdot d_{d}^{-1} \ln \left[\left(1-Y_{s, v, \text { cell }}\right) /\left(1-Y_{s, \text { vsurf }}\right)\right]
$$

Expanding from a single droplet to $\mathrm{Q}_{\mathrm{N}, \text { real }} / \mathrm{Q}_{\mathrm{N}, \text { inj }}$ droplets, the original droplet-vapor source term (see Eq. (4)) needs to be amplified by $\mathrm{Q}_{\mathrm{N}, \text { real }} / \mathrm{Q}_{\mathrm{N}, \mathrm{inj}}$ as follows:

$$
S_{v-d, s}^{(Y)}=\frac{Q_{N, \text { real }}}{Q_{N, \text { inj }}} \int_{t_{i, s \text { start }}}^{t_{\text {starn }}+\Delta t_{f}}\left(\sum_{i=1}^{N_{d, c e l l}}\left(\bar{j}_{s} A_{d}\right)_{i}\right) d t_{d} /\left(V_{\text {cell }} \Delta t_{f}\right)
$$

Accordingly, the numerical calculations of the species deposition in liquid and vapor forms are updated as well:

$$
D F_{s, \frac{l}{v+l}}(t)=\frac{\left(Q_{N, \text { real }} / Q_{N, i n j}\right) m_{s, l}^{(d e p)}(t)}{\left(Q_{N, \text { real }} / Q_{N, i n j)}\right) m_{s, l}^{(i n)}(t)+m_{s, v}^{(i n)}(t)}
$$

and 


$$
D F_{s, \frac{v}{v+l}}(t)=\frac{m_{s, v}^{(d e p)}(t)}{\left(Q_{N, \text { real }} / Q_{N, i n j}\right) m_{s, l}^{(i n)}(t)+m_{s, v}^{(i n)}(t)}
$$

In summary, the underlying assumptions for calculating the present SF-correlation are as follows:

(a) The SF is an averaged function to correct the droplet-vapor interaction difference between actual droplet-injection numbers and assumed injection number.

(b) The correlation is based on numerical experiments considering transport and conversion for $\mathrm{t}=2$ seconds in a single-airway bifurcation.

(c) The SF depends only on the droplet-number density ratio between the realistic and the selected case, presently a sufficient injection droplet-number flow rate of $Q_{\mathrm{N}, \mathrm{inj}}=207$ $[\# /(\mathrm{ml} \mathrm{s})]$.

\section{Numerical Simulations}

\subsection{Geometry and Mesh}

Based on the dimensions provided by Cheng et al. (1999), a somewhat idealized but representative mouth-to-trachea and a triple bifurcation unit (TBU) were selected for the present study (see Fig. 4). Thus this configuration covers the oral cavity to G3 with an $8 \mathrm{~mm}$ mouth-inlet for the electronic cigarette $(\mathrm{EC})$, where the center coordinate of the $\mathrm{EC}$-inlet is $(0,0,0)$. The inhaled airflow is parallel to the positive $\mathrm{y}$-direction and gravity is along the negative $\mathrm{x}$-direction. The bronchial tree lies in the xz-plane.

For the numerical simulation a structured, multi-block, body-fitted hexahedral mesh was developed. The final mesh contained 2,374,679 cells and 2,319,768 nodes. The near-wall cells were refined in element density to resolve any geometric as well as turbulent flow features. The relatively high local mesh resolution is also necessary to accurately calculate values of near-wall derivatives, such as the deposition fluxes. Also, the cells at the boundary of the $8 \mathrm{~mm}$ EC mouthpiece (i.e., mouth inlet) are refined (see Fig. 4).

\subsection{Mesh Independence Test}

Mesh independence tests were performed using steady flow simulations of air with a mouth inlet temperature of $360.15 \mathrm{~K}$ and inlet flow rate of $\mathrm{Q}_{\mathrm{in}}=27.5 \mathrm{ml} / \mathrm{s}$. The wall boundary conditions are set to be no-slip with a constant body temperature of $310.15 \mathrm{~K}$, while the outlets were set to be at uniform pressure. 
The mesh topologies were determined by refining the meshes until grid independence of the flow field solutions was achieved. For example, three meshes were generated with different mesh densities which are shown in Table 4. Specifically, Mesh 2 was refined by factor 2.0 compared to Mesh 1, and Mesh 3 was also refined by factor 2.0. Mesh independence was investigated by comparing non-dimensionalized velocity profiles at a few selected locations (i.e., cross sections A-A', B-B', and C-C') in the flow domain (see Fig. 5 (a)). The nondimensionalized velocity $V^{*}$ is defined as:

$$
V^{*}=\|\vec{V}\| / \bar{V}_{i n}
$$

where $\|\vec{V}\|$ is the local velocity magnitude and $\bar{V}_{\text {in }}=0.5459 \mathrm{~m} / \mathrm{s}$ is the average inlet velocity related to $Q_{\text {in }}=27.5 \mathrm{ml} / \mathrm{s}$.

Considering the velocity profiles at B-B' (i.e., glottis) as an example (see Fig. 5 (b)), differences generated with each mesh are visible. While Mesh 1 is too coarse, the variations of simulated velocity profiles were within $1.0 \%$ between Mesh 2 and the very fine Mesh 3. So, Mesh 2 was employed as the best mesh when considering both accuracy and computational cost.

\subsection{Numerical Setup}

The computational results of the governing equations with appropriate boundary conditions were achieved with a user-enhanced, commercial finite-volume based program, i.e., ANSYS Fluent 15.0 (ANSYS Inc., Canonsburg, PA). The shear stress transport (SST) transition model was selected to solve for the laminar-to-turbulence airflow fields (Zhang and Kleinstreuer, 2011; and Zhang et al., 2012 a \& b; among others). In the CM-P Lab of the MAE Department at NCSU, numerical simulations were performed on a local Dell Precision T7910 workstation (Intel ${ }^{\circledR}$ Xeon ${ }^{\circledR}$ Processor E5-2670 v3 with 2 processors, 12 cores and 64GB RAM), of which 6 to 8 cores were used for the transient inhalation case with the IDR method. Typical run times were approximately 72 to 96 hours. The supplementary user-defined functions (UDFs) are able to perform the following tasks:

(a) Initializing and defining of input variables

(b) Customizing the time step for the discrete phase model (DPM)

(c) Defining realistic transient inhalation waveforms

(d) Recovering Brownian-motion induced droplet velocities

(e) Calculating multicomponent droplet-size change dynamics considering interactions with ambient vapor species 
(f) Defining diffusivities, droplet-vapor interaction source terms, and boundary conditions of the transport equations for vapor species

(g) Calculating and recording local deposition and absorption of EC components in both droplet and vapor forms.

In addition to the UDFs, a MatLab ${ }^{\circledR}$ program for random droplet distributions was implemented at different flow time steps, starting from the mouth inlet.

The flow time step has to be relatively small, i.e., 0.0001 second, which is restricted by the convergence requirements induced by the droplet-vapor interaction calculations. The DPM time step was determined, following Gupta and Peters (1985), i.e.,

$$
\Delta \mathrm{t}_{\mathrm{d}} \approx \frac{3 \pi \mu_{a-v} d_{d, i n i}}{C_{C} m_{d, i n i}}
$$

The rationale for Eq. (32) is as follows:

(a) The DPM time-step should be much larger than the time interval of two successive collisions between droplets and surrounding air molecules; because, the white noise process simulated via the Brownian-motion force is a result of a sufficient number of collisions, i.e., the DPM time step should be much larger than $\tau_{B}$.

(b) The DPM time-step should be approximately equal to or slightly larger than the value indicated by Eq. (32), which is the momentum relaxation time. Actually, the white noise process requires that two successive Brownian-force magnitudes, obtained via a Gaussian random number generator, should be independent of each other. To guarantee that, the DPM time step must be equal to or slightly larger than Eq. (32), so that the energy and momentum the droplets obtained during $\Delta \mathrm{t}$ due to Brownian motion can be damped by the dissipation force.

(c) The DPM time-step should be much less than the large-scale droplet-residence time, because the frequency of Brownian-motion induced fluctuations is much higher so that the number of fluctuations numerically generated during the transport of the droplets should be sufficiently high.

\section{Model Validations}

Basic model validations are discussed in previous papers (i.e., Zhang et al., 2012 a \& b; Feng et al., 2015) and in the next sections. Specifically, to validate the new MCM-DD model, the following should be considered: 
- droplet-size change dynamics due to evaporation/condensation as part of dropletvapor interactions (i.e., phase change);

- discrete phase transport and deposition; and

- vapor species transport and deposition.

\subsection{Droplet-size Dynamics}

As previously reported by Feng et al. (2015), Fig. 6 (a) shows the comparison of the diameter-growth ratios for conventional cigarette smoke particles (CSP) between the present numerical study and experimental measurements by Li and Hopke (1993) at RH=99.5\%. An additional comparison is shown in Fig. 6 (b) between our numerical study and the experimental data of Johnson et al. (2015) for CSPs with initial diameters ranging from $100 \mathrm{~nm}$ to $500 \mathrm{~nm}$ at $\mathrm{RH}=97.5 \%$. As expected, elevated ambient RH-values lead to higher growth ratios for the CSPs with the same initial diameter. Furthermore, with the increase in initial diameter, the growth ratio increases for CSPs under the same ambient RH. These observations were also confirmed by Johnson et al. (2015).

\subsection{Discrete Phase Transport and Deposition}

Validations of the discrete phase transport and deposition model (i.e., particle deposition fractions), based on the Euler-Lagrange method, are documented in our previous publications (Zhang et al., 2004, 2005, \& 2012 a).

\subsection{Vapor Species Transport and Deposition Model}

For the validation of the multicomponent vapor species transport and deposition model (see Fig. 7 (a)), methyl tertiary-butyl ether (MTBE) and ethanol transport and deposition were compared to the benchmark numerical results of Zhang et al. (2006). The oral cavity had a 20$\mathrm{mm}$ mouth opening with the extended trachea approximately $14 \mathrm{~cm}$ from the outlet to the glottis. The final hybrid tetrahedral/pentahedral mesh was generated with six prism layers near the wall surface. The total number of elements was 999,760. The mouth inlet flow rates, Qin, varied from $15 \mathrm{~L} / \mathrm{min}$ to $60 \mathrm{~L} / \mathrm{min}$. The non-dimensionalized vapor mass fractions at the mouth inlet are 1.0 for both MTBE and Ethanol. The details of wall absorption rate boundary condition are discussed in Sect. 2.3.2. The SST transition model was selected for simulating the laminar-toturbulence airflow, where residual constraints were set at 1e-4 for continuity, momentum, energy, and turbulent flow equations, while 1.0e-6 was set for the vapor-mass transport equations. Figure 7 (b) shows the comparison of deposition fractions of MTBE and ethanol vapors in the oral 
cavity for different inlet flow rates $\left(\mathrm{Q}_{\mathrm{in}}=15,30\right.$, and $\left.60 \mathrm{~L} / \mathrm{min}\right)$. The small differences between the current simulation and the result of Zhang et al. (2006) are due to the following reasons:

- Different models for laminar-to-turbulence transition flow were employed.

- A different length for the trachea extension was employed.

In summary, these validations instill confidence that the MCM-DD model is accurate enough to numerically simulate vapor transport and absorption in human lung airways.

\section{Results and Discussion}

\subsection{Prediction of Liquid-Vapor Interaction on Compound Deposition}

The significance and hence necessity to include liquid-vapor interaction mechanisms for the prediction of total deposition fractions (TDFs) was investigated. Specifically, two transient cases in the idealized mouth-to-G3 geometry (see Fig. 4) were simulated with and without liquid-vapor interactions, i.e., phase change due to evaporation or condensation effects. Figure 8 depicts (see Table 1) significant TDF differences for PG, glycerol and nicotine between the two cases. Specifically, approximately $7 \%$ of glycerol, $29 \%$ of PG, and $17 \%$ of nicotine deposit less when considering liquid-vapor interactions. In reality all three vapor species condensate during transport, i.e., more of the compounds will be transferred to liquid form and hence moving with the droplets. That leads to lower TDFs in vapor form (see Eq. (23)) because of the lower local vapor concentrations. Additionally, with liquid-vapor interaction, the TDFs in liquid form (see Eq. (22)) are slightly lower due to the combined influence from the increased inertia of the growing droplets and the higher species mass in a single droplet. The comparison in Fig. 8 also indicates that with the increase in species volatility and initial deviation from the equilibrium partition between liquid and vapor form, including the liquid-vapor interaction mechanism becomes very important for accurate TDF predictions.

\subsection{EC-aerosol Transport and Deposition under Transient Puffing/Inhalation Conditions}

The puffing/inhalation waveform \#1 (Fig. 1 (a)) is based on measurements of EC inhalation behavior (Lee et al., 2015; Behar et al., 2015; and Farsalinos et al., 2013). As discussed, we selected waveform \#1 with puff duration 2.4 seconds and puff volume $63.9 \mathrm{ml}$, as a representative inhalation scenario (see initial setup shown in Table 1). The regional and total deposition data of PG, glycerol, and nicotine after $\mathrm{t}=2.4 \mathrm{~s}$ are listed in Table 5 . At the end of the single EC puff $(\mathrm{t}=2.4 \mathrm{~s}), 10.57 \%$ of glycerol, $16.53 \% \mathrm{PG}$ and $11.95 \%$ of nicotine deposited in the upper RT-region from mouth to G3. 


\subsubsection{Transient Airflow Structures and Vortices Visualization during a Single EC Puff Cycle}

As the transient airflow structures determine EC droplet-vapor transport and deposition, a detailed analysis of the local airflow patterns is very important. Figures 9 and 10 depict an inlet jet, stagnation point flow, recirculating flow, and secondary flows. The relationship between the airflow field and the one-way coupled transport and deposition patterns of EC aerosols are discussed next.

\section{$\underline{\text { Transient airflow structures }}$}

Figures 9 (a) to (g) show the airflow field evolution at the sagittal plane $(\mathrm{Z}=0)$ for the transient puff waveform \#1, colored by the velocity magnitude $\|\vec{V}\|$. At $\mathrm{t}=0.1$ s (see Fig. 9 (a)), the inlet flow rate starts to increase and a jet stream begins to form. While the developing jet does not impact the lower palate, there are high velocity regions in contracting cross sections such as the connection between oral cavity and pharynx as well as the glottis. At $t=0.2 \mathrm{~s}$ (see Fig. 9 (b)), with the increase in air-drawing intensity, the inlet jet is well formed, impacting the lower palate. The jet core deviates due to impaction and extends to the back of the pharynx. The pressure difference induced by the velocity difference inside and outside the jet core causes the formation of recirculation regions, as seen near the upper palate, lower palate and the inner region of the pharynx (see red-dash circles in Figs. 9 (b) and (c)). Clearly, droplets suspended in those recirculation regions have a longer residence time and will less likely deposit. At $t=0.4 \mathrm{~s}$, the air-drawing intensity has reached its maximum (actually $47 \mathrm{ml} / \mathrm{s}$ at $\mathrm{t}=0.3 \mathrm{~s}$ ) and the jet-core extends into the trachea and bends towards the front side. Another recirculation region is formed at the backside of the trachea (see the red-dash circles in Fig. 9 (c)). As the air-drawing intensity decreases after $\mathrm{t}=0.6 \mathrm{~s}$ (see Figs. 9 (d) to (g)), the jet-core velocity decreases. It is of interest to observe the fluctuating interface between the jet-core and the recirculation region in the trachea (see the red-dash circles in Figs. 9 (d) and (e)). This special flow pattern was only observed for the transient waveform (see Fig. 1(a)) and does not appear under steady inhalation conditions. Similar jet stream and recirculating flow characteristics were reported by Feng and Kleinstreuer (2011) with a subject-specific human upper lung airway model (mouth to G9). So, the idealized human upper lung airway geometry can be considered to be a representative geometry for capturing major airflow structures. 


\section{Vortex visualization}

Velocity contours alone (see Figs. 9 (a) to (g)) are not sufficient to fully depict all airflow structures, especially 3-D vortices and/or secondary flows which cause deposition of inhaled aerosol. Vortices need to be quantitatively identified based on a function that can be evaluated "point-by-point" in order to determine if each flow-field point is inside or outside of the vortex structure. Different vortex identification methods were compared by Jiang et al. (2005) and Roth (2000), where the helicity is defined as:

$$
H=\vec{v} \cdot \vec{\omega}
$$

Degani et al. (1990) proposed the "helicity method" which utilizes non-dimensionalized helicity, $\widetilde{H}$, for extracting vortex core lines as:

$$
\widetilde{H}=\frac{\vec{v} \cdot \vec{\omega}}{|\vec{v}||\vec{\omega}|}
$$

where $\widetilde{H}$ is the cosine of the angle between local velocity $\vec{v}$ and vorticity $\vec{\omega}$ (the curl of $\vec{v}$ ) . Degani et al. (1990) proposed that the vortex-core line (or axis) is a curve where the vorticity $\vec{\omega}$ tends to align with the velocity $\overrightarrow{\mathrm{v}}$, i.e., $\widetilde{\mathrm{H}} \approx \pm 1$. Furthermore, the sign of $\widetilde{H}$ indicates the direction of the vortex (clockwise or counterclockwise) with respect to the streamwise velocity vector.

Using the helicity method, the major vortex structures are visualized at $\mathrm{t}=0.4 \mathrm{~s}$ when the inlet jet-core has reached the first bifurcation (see Fig. 10). As shown in Fig. 10, several pairs of vortices are generated due to the high-velocity jet in the oral cavity near the mouth inlet. Before entering the contraction between the oral cavity and pharynx, Dean-type vortices swirl towards the upper palate near the wall and towards the lower palate near the sagittal plane $(\mathrm{Z}=0)$. Entering the pharynx, glottis and trachea, vortex structures remain and intertwine. Double vortices can also be observed near each bifurcation point from G1 to G3. Such local Dean-type vortices were also observed for steady airflow structures in human lung airway bifurcations (Feng, 2013). These vortices are capable of enhancing the local heat and mass transfer in the human respiratory system; thus, influencing local EC-aerosol transport and deposition.

\subsubsection{Temperature Field}

The evolution of the temperature field when using the transient puff waveform \#1 is shown in Figs. 11 (a) to (g). Upstream to the location where the inlet jet impacts the lower palate, the high-temperature core $(\mathrm{T}=360.15 \mathrm{~K})$ is concentrated without much diffusion. In contrast, jet- 
stream impaction onto the lower palate leads to more complex secondary flow patterns (see Figs. 9 (a) to (g) and Fig. 11), which enhances local heat transfer. As a result, during the whole puff cycle the temperature is more evenly distributed in the pharynx (see Figs. 11 (a) to (g)). Furthermore, the local temperature distribution during puffing in the human upper lung airway from mouth to G1 varies; hence, it will influence condensation or evaporation and thereby leading to different droplet-size changes as well as local vapor concentrations and compound depositions.

\subsubsection{Species Transport and Deposition Patterns in Vapor Form}

Given the initial liquid-vapor partitions shown in Table 1, all three species, i.e. glycerol, PG and nicotine, undergo continuous condensation. Hence, local vapor transport patterns among the three species are similar and so only local nicotine-vapor transport and deposition at different time steps has been tracked, being the most important of the compounds (see Figs. 12 to 14). The regional and total deposition data are listed in Table 5 and visualized in Fig. 20. Nondimensionalized vapor mass fraction $\widetilde{Y}_{s, v}$, depicted in Figs. 12 and 13, is calculated as:

$$
\tilde{Y}_{s, v}=Y_{s, v} / Y_{s, v}^{(i n)}
$$

where $Y_{s, v}^{(i n)}$ is the nicotine-vapor mass fraction at the mouth inlet.

Compared to the airflow field structure at different time steps (see Figs. 9 (a) to (g) and Fig. 10), nicotine-vapor transport is determined by both convection and diffusion, where the local concentration is also influenced by condensation/evaporation between droplets and vapors. At $\mathrm{t}=0.1 \mathrm{~s}$ (see Figs. 12 (a) and 13 (a)), nicotine-vapor follows the air-inlet jet with some diffusion, similar to the temperature (see Fig.11 (a)). At $t=0.2 \mathrm{~s}$, just after the inlet jet impacts the lower palate, higher concentrations of nicotine vapor exist closer to the lower palate (see the red dashed circle in Fig. 13 (b)) where the double-vortex in the oral cavity enhances mixing of the high concentration nicotine vapor with the low concentration nicotine vapor near the upper palate (see Fig. 13 (b)). From t=0.3s to t=0.5s (see Figs. 12 (c) to (f) and Figs. 13 (c) to (f)) reaching the first bifurcation, the distribution of the nicotine-vapor mass fraction at different cross sections follows the local secondary flow structures (see Fig. 10). Specifically, the recirculating flow (see Figs. 9 (c)) enhances nicotine-vapor mixing in the pharynx (see Figs. 12 (d)). The jet core towards the front of the trachea (see Figs. 9 (c) and (d)) also carries highconcentration nicotine vapor in the same direction (see Figs. 12 (d) and (e)). Later on, nicotine 
vapor becomes more and more evenly distributed from mouth to G3 due to the secondary-flow induced mixing and diffusion effects (see Figs. 12 (g) and (h) and Figs. $13(\mathrm{~g})$ and (h)).

Figures 14 (a) to (g) show the accumulated nicotine-vapor deposition surface density

$\rho_{A}^{(d e p)}\left[\mathrm{kg} / \mathrm{m}^{2}\right]$ at different time levels. As expected, nicotine vapor deposits gradually from the mouth to G3, while the transport and deposition downstream experiences a delay. Enhanced deposition occurs near the EC exit (or mouth inlet), lower palate, anterior part of the oral cavity, the outside bend of the pharynx, and bifurcation points. This is due to the large concentration gradients induced by direct impaction or long residence times of nicotine vapor in recirculation regions with complicated secondary flow structures.

\subsubsection{Species Transport and Deposition Patterns in Liquid Form with Droplet Size Change Dynamics}

The transport and deposition of EC-smoke constituents in liquid form is associated with the nanodroplet dynamics, i.e., transport, size change and deposition, as shown in Figs. 15 (a) to (f) and Figs. 16 (a) and (b). Droplets are colored by its instantaneous diameter which is influenced by the condensation/evaporation effects interacting with the ambient vapor phases.

For the EC-droplet transport and size dynamics shown, the suspended EC droplets with $\mathrm{d}_{\mathrm{d}, \mathrm{ini}}=400 \mathrm{~nm}$ keep absorbing vapors from the ambient and hence growing in size. Some droplets are able to follow closely the air-jet, as can be observed when considering the share of droplets forming the stream's frontier. Meanwhile, other droplets escape from the mainstream due to the combined effects of Brownian motion and local secondary flows, thereby aggregating near the lung-airway surfaces where the air velocity is low. Thus, before the glottis at the same crosssectional plane perpendicular to the airway centerline, droplets closer to the center have smaller diameters because of the relatively low residence time due to the locally higher velocity. After the contraction in the glottis, the same effect is not obvious. It is worth mentioning that at $\mathrm{t}=0.5 \mathrm{~s}$, the frontier of the droplets reaches $\mathrm{G} 1$, and at $\mathrm{t}=0.8 \mathrm{~s}$ it reaches $\mathrm{G} 3$. Additionally, at $\mathrm{t}=0.6 \mathrm{~s}$ and $\mathrm{t}=0.8 \mathrm{~s}$ (see Figs. 15 (c) and 15 (d)), due to the lower pressure near the inner wall downstream of the first bifurcating point, droplets are driven towards the inner wall. However, as time evolves, some droplets also escape and get suspended in the region closer to the outer wall (Figs. 15 (e) and 15 (f)). The maximum growth ratio at the end of the single puff cycle $(\mathrm{t}=2.4 \mathrm{~s})$ is about 2.18. Figures 16 (a) and (b) show the local deposition patterns of droplets at the end of the single puff and after one-second holding, colored by their final diameter. 
Multiple deposition mechanisms determine different deposition patterns from mouth to G3. Favorable spots for droplet deposition include the lower palate, back of the pharynx, glottis and regions near each bifurcation point. Droplets with low residence times experience relatively small growth rates and deposit typically via direct impaction, as in the oral cavity. However, there are other droplets deposited in the oral cavity with somewhat larger size. Such depositions occur because of Brownian motion and secondary flow effects, i.e., they had longer residence times and thereby more time to absorb ambient vapors to grow larger.

\subsection{Parametric Sensitivity Analyses: Impact of Puffing/Inhalation Patterns on EC Aerosol Deposition}

The impacts of different steady and transient inhalation patterns on aerosol deposition were simulated. Table 3 and Figs 1 (a), (b) show several puff volumes and durations studied here.

\subsubsection{Inhalation Flow Rate}

The impact of inhalation flow rate on EC-aerosol transport and deposition was investigated by first fixing the steady puffing duration at different flow rates (i.e., $\mathrm{Q}_{\text {in }}=12,20$, and $27.5 \mathrm{ml} / \mathrm{s}$ at $\mathrm{t}=2 \mathrm{~s}$ ) and then fixing the total puff volume (i.e., $27.5 \mathrm{ml} / \mathrm{s}$ at $\mathrm{t}=2 \mathrm{~s}$ and $18.33 \mathrm{ml} / \mathrm{s}$ at $\mathrm{t}=3 \mathrm{~s}$ ). Histograms of regional and total deposition fractions (RDFs and TDFs) of glycerol, PG and nicotine in both vapor and liquid forms for the four cases are given in Figs. 17 to 19.

\section{Fixed puff duration $(t=2 \mathrm{~s})$ at different flow rates $\left(Q_{\text {in }}=12,20\right.$, and $\left.27.5 \mathrm{ml} / \mathrm{s}\right)$}

Overall, absorption of all three species in vapor form (see Figs. 17 (a), 18 (a) and 19 (a)), being less than in liquid form, has in all regions a consistent upward trend with increasing flow rate as recorded after $\mathrm{t}=2 \mathrm{~s}$. In contrast, there are no consistent regional correlations between absorption values for PG, glycerol and nicotine in liquid form and the magnitudes of inhalation flow rates (see Figs. 17 (b), 18 (b), and 19 (b)), resulting in almost uniform total deposition efficiencies for all three flow rates after $\mathrm{t}=2 \mathrm{~s}$.

Specifically, for species deposition in vapor form shown in Figs. 17 (a), 18 (a), and 19(a) the following observations can be made:

- In the oral cavity, oropharynx, trachea and B1 to B3, vapor absorption fractions $D F_{s, \frac{v}{v+l}}$ increase after two seconds with higher inlet flow rates. This is mainly due to direct impaction and secondary flows, as regionally a lower inhalation flow rate implies a longer dropletresidence time, providing extra time for the species in vapor form to condensate. This also explains, at least for the oral-cavity, the opposite trend of nicotine deposition in liquid form (see Figs. 17 (b), 18 (b), and 19 (b)). Lower flow rates also delay the convective transfer of 
vapor into lower lung airways, which is another mechanism to influence the local vapor concentration and absorption rate.

- For a fixed inhalation duration of, say, $\mathrm{t}=2 \mathrm{~s}$, stronger drawing (e.g., $27.5 \mathrm{ml} / \mathrm{s}$ ) will result in a more evenly distributed nicotine vapor absorption, $D F_{s, \frac{v}{v+l}}$, from the oral cavity to B3.

- Simultaneously, stronger drawing (e.g., $27.5 \mathrm{ml} / \mathrm{s}$ ) will induce higher TDFs for all vapor species.

Concerning species deposition in liquid form, as shown in Figs. 17 (b), 18 (b) and 19 (b), one can conclude the following:

- In the oral cavity, the species regional deposition fractions (RDFs) in liquid form, $D F_{s, \frac{l}{v+l}}$, after $\mathrm{t}=2 \mathrm{~s}$ decrease with higher inlet flow rates as the result of several mechanisms, i.e.,

- at lower inlet flow rates, the longer residence times for droplets in the oral cavity lead to increased chances for deposition due to Brownian motion, secondary flows, and sedimentation;

- a longer transport time before reaching the lower palate also increases the hygroscopic growth of droplets, thereby strengthening direct impaction; although, the inlet flow rates are still relatively low.

- In the oropharynx, no monotonic trend in RDFs can be observed with increased inlet flow rates, as different deposition mechanisms are in effect.

- In the trachea and B1 to B3, the RDFs after $\mathrm{t}=2 \mathrm{~s}$ increases with higher inlet flow rates, due to stronger Brownian motion with less hygroscopic growth, stronger direct impaction, and secondary flow effects.

- Again, if the inhalation duration is fixed, i.e., $\mathrm{t}=2 \mathrm{~s}$, stronger drawing (e.g., $27.5 \mathrm{ml} / \mathrm{s}$ ) will induce a more evenly-distributed species absorption from the oral cavity to B3.

- The selected range of inhalation flow rates (from $12 \mathrm{ml} / \mathrm{s}$ to $27.5 \mathrm{ml} / \mathrm{s}$ ) has no significant impact on the species' total deposition fractions (TDFs), and no monotonic trend can be observed due to the different RDF occurrences.

For the total species depositions depicted in Figs. 17 (c), 18 (c) and 19 (c), the following can be noted:

- Due to the higher DF-differences of all species in liquid form than in vapor form, the impact of flow rate on $D F_{S}$ has the same trend as the RDF-trends in liquid form, i.e., $D F_{s, \frac{l}{v+l}}(t)$. 
- If the inhalation duration is fixed, i.e., $\mathrm{t}=2 \mathrm{~s}$, stronger drawing (e.g., $27.5 \mathrm{ml} / \mathrm{s}$ ) will induce more evenly-distributed species absorption from the oral cavity to B3.

- The inhalation flow rate (from $12 \mathrm{ml} / \mathrm{s}$ to $27.5 \mathrm{ml} / \mathrm{s}$ ) has no significant impact on the total deposition fractions of all species in the upper lung airways from mouth to G3.

Fixed total puff volume $(55 \mathrm{ml})$ with different puff flow rates $\left(Q_{\text {in }}=27.5 \mathrm{ml} / \mathrm{s}\right.$ and $\left.18.33 \mathrm{ml} / \mathrm{s}\right)$

To further investigate the influence of inhalation waveform on species transport and deposition, two different puffing conditions with the same total puff volume (i.e., $55 \mathrm{ml}$ ) were simulated and compared (Figs. 17 to 19). With the same puff volume, lower air-drawing intensity, e.g., $18.33 \mathrm{ml} / \mathrm{s}$, induces higher RDFs in all regions, i.e., from the mouth to $\mathrm{G} 3$ for the following reasons:

- Longer vapor residence time induces higher vapor absorption/deposition.

- Longer droplet residence time induces a higher probability of deposition.

It can also be observed that in the four cases investigated, all three species are fast absorbed in the oral cavity and oropharynx because of the longer vapor and droplet residence times as well as larger airway-surface areas. Furthermore, except for the absorption coefficient, the initial liquid-vapor partitions and volatilities also influence the deposition of vapors. Specifically, glycerol deposition in vapor form is negligible compared to its deposition in liquid form because of the initial liquid-vapor partition containing only $0.1293 \%$ glycerol vapor due to the low volatility. In contrast, PG deposition in vapor form is comparable to the deposition in liquid form, which is due to the initial partition based on its relatively high volatility.

\subsubsection{Influence of Holding Duration on Deposition for Transient Waveform \#1}

Most publications focusing on smoke-aerosol deposition in human lung models did not include the holding phase after puffing. To further investigate the influence of holding duration on deposition, results from two scenarios were compared, i.e., waveform \#1 (see Fig. 1 (a)) after $\mathrm{t}=2.4 \mathrm{~s}$ and with one-second holding after $\mathrm{t}=3.4 \mathrm{~s}$. Nicotine deposition data are shown in Figs. 20 (a) to (c), representing also the trends of the other two species (i.e., glycerol and PG). It is obvious that the extra one-second holding increases the regional and total depositions of nicotine in both vapor and liquid forms. Indeed, a longer holding phase after puffing will result in higher depositions due to vapor diffusion and some droplets already near the airway walls. Considering the droplet deposition pattern shown in Figs. 16 (a) and (b), with the one-second holding the average droplet growth ratio is higher and so is the number of droplets deposited. 


\subsubsection{Transient Waveform \#1 (CC-users) vs. Transient Waveform \#2 (EC-users)}

Waveforms \#1 vs. waveform \#2 represents contrasting EC-puffing behavior by a conventional cigarette user and an EC-user for the same total puff volume (i.e., $63.9 \mathrm{ml}$ ), but different puff durations and peak flow rates (Figs. 1(a) and 1(b)). The comparisons of nicotine deposition in vapor and liquid forms are also shown in Figs. 20 (a) to (c). Although Fig. 20 (c) still indicates that if the total puff volume is fixed, waveform \#2 with a lower puff intensity will induce higher total deposition, i.e., the RDFs in vapor or liquid forms are not monotonically increasing or decreasing. The results differ from the steady-state puffing outcome (see Sect. 5.3.1). The complexity is introduced by the transient airflow fields, as they have more variations in local secondary flows, resulting in a more complex deposition scenario of droplets as well as local vapor absorption.

In summary, EC-users who are accustomed to EC-products have a puffing behavior (i.e., waveform \#2) which leads to elevated nicotine uptakes in the upper lung airways when compared to CC-users with waveform \#1.

\subsection{Parametric Sensitivity Analyses: Impact of Inhalation Patterns on EC Liquid-Vapor \\ Interaction}

To provide more physical insight on liquid-vapor interactions, comparisons of vaporliquid mass change percentages for the three compounds as well as droplet-growth ratios were evaluated for different puffing patterns. The vapor-liquid mass change percentage of the s-th species $\beta_{v-l, s}(\mathrm{t})$ is:

$$
\beta_{v-l, s}(\mathrm{t})=\frac{m_{s, v}^{(i n)}-\left(m_{s, v}^{(d e p)}+m_{s, v}^{(s u s)}+m_{s, v}^{(o u t)}\right)}{m_{s, v}^{(i, v}} \times 100 \%
$$

where $m_{s, v}^{(i n)}, m_{s, v}^{(s u s)}, m_{s, v}^{(d e p)}$ and $m_{s, v}^{(o u t)}$ are the accumulated inflow mass, suspended mass, deposition mass, and outflow mass of vapor species. A negative value of $\beta_{v-l, s}(t)$ indicates net evaporation, while a positive value implies net condensation. The droplet-growth ratio (GR) at time $\mathrm{t}$ is:

$$
\mathrm{GR}(\mathrm{t})=\frac{d_{d}(t)}{d_{d, \text { ini }}}
$$

For all three species, i.e., glycerol, PG and nicotine, under the steady two-second-puff condition, Fig. 21 (a) indicates that a lower inlet flow rate will cause a higher vapor-liquid mass transfer percentage. This is probably due to the longer droplet residence time allowing for extended 
droplet-vapor interactions. Figure 21 (a) also shows a comparison among the three transient puffing cases. It can be observed that the additional one-second holding yields a slightly higher mass transfer percentage, which is also due to the longer droplet residence time. With the fixed total puff volume (i.e., $63.9 \mathrm{ml}$ ), lower inhalation intensity case (see waveform \#2) provides higher mass change percentages for all three species.

Figure 21 (b) illustrates the size-change dynamics of suspended droplets $\left(d_{d, i n i}=400 \mathrm{~nm}\right)$ under different puffing conditions. Due to the continuous condensation effect, the average droplet GR gradually increases with time for all puffing scenarios. For steady puffing, combined with the observations given in Fig. 21 (a), a lower inlet flow rate induces a higher mass transfer percentage, i.e., a stronger condensation effect, thereby leading to a higher average droplet GR than the stronger inhalation case. The increasing GR-rate (i.e., the slope of the curve) gradually decreases, indicating the liquid-vapor interactions gradually reach a quasi-equilibrium state. However, for the transient puffing case shown in Fig. 21 (b), the increasing GR-rate is not monotonic with time. This is because of the variation in inhalation flow rate during the transient puffing cycle. Specifically, after 2.4s holding starts and no air and EC aerosols are being further inhaled until 3.4 seconds. The average droplet-GR increases at a faster rate because of the increasing RH in the lung airways and the near-static airflow field exhibiting weak convection.

\section{Conclusions}

A novel CF-PD model, based on the MCM-DD approach, has been developed which can simultaneously simulate transport and deposition of interactive, multicomponent droplet-vapor mixtures in human lung-airways. At present, the focus is on two-phase EC-nicotine absorption in an idealized human upper airway model, subject to different puffing/inhalation scenarios. Because of the high particle concentration, a scale-factor correlation was introduced, as part of the IRD method, to achieve realistic deposition data. It should be noted that the MCM-DD model can be extended to other components to evaluate potential health risks of additional constituents present in EC-smoke. Other applications of the model include analysis of propulsion fuels and drug-aerosols.

Selected EC-aerosol compositions are listed in Table 1 for realistic computer inputs. With the comparisons of deposition and interaction results under different EC-smoking modes, the major conclusions are as follows: 
(a) Complex secondary flows and Dean-type vortices exist throughout the idealized mouthto-G3 human upper lung airway model which significantly influence droplet transport and distribution. It is of interest to note that the major flow patterns captured in the idealized human upper lung airways appear also in subject-specific models.

(b) Droplet-vapor interaction mechanisms must be considered, as they have a strong impact on deposition predictions of inhaled EC aerosols.

(c) EC-aerosol deposition mechanisms includes direct impaction, Brownian motion, secondary flows, recirculating flows with enhanced residence times, and gravitational sedimentation.

(d) At a fixed inhalation duration (e.g., $\mathrm{t}=2 \mathrm{~s}$ ) for steady puffing, stronger air-drawing will induce more evenly distributed depositions in the mouth-to-G3 region, less vapor-liquid mass transfer percentages, and lower average droplet-growth ratios.

(e) For a fixed puff volume at steady inhalation, stronger drawing will lead to lower regional and total depositions in both vapor and liquid forms for all species. However, for the two transient waveforms (see Figs. 1 (a) and (b)), stronger drawing will not induce monotonic changes in all regional depositions, but it will still induce lower total depositions.

(f) Longer droplet-vapor holding after puffing results in to higher deposition fractions, higher vapor-liquid mass transfer percentages, and higher average droplet-growth ratios. Hence, for accurate uptake predictions, it is necessary to simulate not only the puffing/inhalation process but also the holding action.

\section{Future Work}

In light of the necessary simplifications made and the current lack of experimental deposition data for E-cigarettes, improvements are suggested as follows:

(a) Development of a generalized SF-function with extended dependence on droplet diameter, droplet composition, liquid-vapor partition, RH, and lung-airway geometry.

(b) Further investigation of inter-subject variability using different models of human respiratory systems.

(c) Additional parametric sensitivity analyses with excessive compound concentrations, presence of variable mucus layers, different aerosol-inhalation waveforms, ECaerosols vs. CC-smoke particles, second-hand smoke inhalation, etc. 
(d) Adjustment of model-parameter/coefficient values and hence impact evaluation as new experimental data sets appear. Of special interest are:

- Inhalation/puffing waveforms for ECs

- Reliable saturation pressure measurements for PG, glycerol, nicotine, formaldehyde, flavor chemicals, etc.

- Liquid-vapor partitions for PG, glycerol, nicotine, and other species of interest

- Activity coefficients of all species in EC multicomponent droplets

- Compound parameter values, e.g., absorption coefficients between air and mucus layer.

(e) Consideration of cloud-formation/motion effects, charge effects, and coagulation

\section{Acknowledgements}

The authors are grateful for the financial support of Y. F. by a grant from NSF (CBET1232988), Environmental Health and Safety of Nanotechnology, and by Altria Client Service

LLC (ALCS). Insightful discussions with Dr. Pithawalla from ALCS (Richmond, VA) are appreciated as well. 


\section{References}

Alderman, S. L., Song, C., Moldoveanu, S. C., \& Cole, S. K. Particle Size Distribution of ECigarette Aerosols and the Relationship to Cambridge Filter Pad Collection Efficiency. Beiträge zur Tabakforschung/Contributions to Tobacco Research, 26(4), 183-190.

Appleton, S., Liu, J., Lipowicz, P. J., \& Sarkar, M. (2015). Effect of cigarette design on biomarkers of exposure, puffing topography and respiratory parameters. Inhalation Toxicology, 27(3), 1-7.

Behar, R. Z., Hua, M., \& Talbot, P. (2015). Puffing Topography and Nicotine Intake of Electronic Cigarette Users. PloS one, 10(2), e0117222.

Canada. (2014). Canada Government Tobacco Act: Tobacco Reporting Regulations, SOR/2000273, Part 3: Emissions from designated tobacco products. Health Canada.

Cheng, Y. S., Zhou, Y., \& Chen, B. T. (1999). Particle deposition in a cast of human oral airways. Aerosol Science and Technology, 31(4), 286-300.

Cunningham, A., Clayford, S., Vas, C., Costigan, S. (2015), Development \& validation of a device to measure e-cigarette users' puffing topography, Global Forum of Nicotine 2015 (GFN2015), Warsaw, Poland.

Degani, D., Seginer, A., \& Levy, Y. (1990). Graphical visualization of vortical flows by means of helicity. AIAA Journal, 28(8), 1347-1352.

Eatough, D. J., Benner, C. L., Bayona, J. M., Richards, G., Lamb, J. D., Lee, M. L., Lewis, E., \& Hansen, L. D. (1989). Chemical composition of environmental tobacco smoke. 1. Gasphase acids and bases. Environmental science \& technology, 23(6), 679-687.

Evans, S. E., \& Hoffman, A. C. (2014). Electronic cigarettes: abuse liability, topography and subjective effects. Tobacco Control, 23(suppl 2), ii23-ii29.

Fahy, J. V., \& Dickey, B. F. (2010). Airway mucus function and dysfunction.New England Journal of Medicine, 363(23), 2233-2247.

Farsalinos, K. E., Spyrou, A., Tsimopoulou, K., Stefopoulos, C., Romagna, G., \& Voudris, V. (2014). Nicotine absorption from electronic cigarette use: comparison between first and new-generation devices. Scientific Reports, 4.

Farsalinos, K. E., Kistler, K. A., Gillman, G., \& Voudris, V. (2015). Evaluation of electronic cigarette liquids and aerosol for the presence of selected inhalation toxins. Nicotine \& Tobacco Research, 17(2), 168-174.

Feng, Y. "Non-spherical particle dynamics analysis with applications to inhaled aerosol transport and deposition in human upper airway models," Ph.D. dissertation (NC State University, Raleigh, NC, 2013) 
Feng, Y., Kleinstreuer, C. (2011). Computational analysis of droplet evaporation and deposition in a realistic respiratory tract subject to puff-like inhalation waveforms, $2^{\text {nd }}$ International Conference on Computational \& Mathematical Biomedical Engineering (CMBE11), Washington D. C., USA.

Feng, Y., Kleinstreuer, C., \& Rostami, A. (2015). Evaporation and condensation of multicomponent electronic cigarette droplets and conventional cigarette smoke particles in an idealized G3-G6 triple bifurcating unit. Journal of Aerosol Science, 80, 58-74.

Gowadia, N., \& Dunn-Rankin, D. (2010). A transport model for nicotine in the tracheobronchial and pulmonary region of the lung. Inhalation toxicology, 22(1), 42-48.

Gupta, D., \& Peters, M. H. (1985). A Brownian dynamics simulation of aerosol deposition onto spherical collectors. Journal of Colloid and Interface Science, 104(2), 375-389.

Hayduk, W., \& Laudie, H. (1974). Prediction of diffusion coefficients for nonelectrolytes in dilute aqueous solutions. AIChE Journal, 20(3), 611-615.

Hinds, W. C. (1999). Aerosol Technology: Properties, Behavior, and Measurement of Airborne Particles ( $2^{\text {nd }}$ edition). John Wiley \& Sons: New York, USA

ICRP (1994). Human Respiratory Tract Model for Radiological Protection. ICRP Publication 66. Ann. ICRP 24 (1-3).

Jiang, M., Machiraju, R., \& Thompson, D. (2005). Detection and Visualization of. The Visualization Handbook, 295.

Johnson, T. J., Olfert, J. S., Yurteri, C. U., Cabot, R., \& McAughey, J. (2015). Hygroscopic effects on the mobility and mass of cigarette smoke particles. Journal of Aerosol Science. $86,69-78$.

Keyhani, K., Scherer, P. W., \& Mozell, M. M. (1997). A numerical model of nasal odorant transport for the analysis of human olfaction. Journal of Theoretical Biology, 186(3), 279-301.

Kleinstreuer, C. \& Zhang, Z. (2003). Laminar-to-turbulent fluid-particle flows in a human airway model. Int. J. Multiphase Flow, 29, 271-289.

Kleinstreuer, C., \& Feng, Y. (2013). Lung deposition analyses of inhaled toxic aerosols in conventional and less harmful cigarette smoke: a Review. International Journal of Environmental Research and Public Health, 10(9), 4454-4485.

Lee, Y. H., Gawron, M., \& Goniewicz, M. L. (2015). Changes in puffing behavior among smokers who switched from tobacco to electronic cigarettes. Addictive Behaviors, 48, 14.

Li, W., \& Hopke, P. K. (1993). Initial size distributions and hygroscopicity of indoor combustion aerosol particles. Aerosol Science and Technology, 19(3), 305-316. 
Luchtel, D. L. (1978). The mucous layer of the trachea and major bronchi in the rat. Scand. Electr. Micro, 2, 1089-1098.

Manigrasso, M., Buonanno, G., Stabile, L., Morawska, L., \& Avino, P. (2015 a). Particle doses in the pulmonary lobes of electronic and conventional cigarette users. Environmental Pollution, 202, 24-31

Manigrasso, M., Buonanno, G., Fuoco, F. C., Stabile, L., \& Avino, P. (2015 b). Aerosol deposition doses in the human respiratory tree of electronic cigarette smokers. Environmental Pollution, 196, 257-267.

Offermann, F. J. (2015). Chemical emissions from e-cigarettes: Direct and indirect (passive) exposures. Building and Environment.

Pankow, J. F. (2001). A consideration of the role of gas/particle partitioning in the deposition of nicotine and other tobacco smoke compounds in the respiratory tract. Chemical research in toxicology, 14(11), 1465-1481.

Roth, M. (2000). Automatic Extraction of Vortex Core Lines and Other Line Type Features For Scientific Visualization. Hartung-Gorre.

Schmehl, R., Rosskamp, H., Willmann, M., \& Wittig, S. (1999). CFD analysis of spray propagation and evaporation including wall film formation and spray/film interactions. International Journal of Heat and Fluid Flow, 20(5), 520-529.

Schroeter, J. D., Asgharian, B., Price, O. T., Kimbell, J. S., Kromidas, L., \& Singal, M. (2016). Simulation of the Phase Change and Deposition of Inhaled Semi-volatile Liquid Droplets in the Nasal Passages of Rats and Humans. Journal of Aerosol Science, 95, 15-29.

Shi, H., Kleinstreuer, C., Zhang, Z., \& Kim, C. S. (2004). Nanoparticle transport and deposition in bifurcating tubes with different inlet conditions. Physics of Fluids, 16(7), 2199-2213.

Talih, S., Balhas, Z., Eissenberg, T., Salman, R., Karaoghlanian, N., El Hellani, A., Baalbaki, R., Saliba, N., \& Shihadeh, A. (2014). Effects of user puff topography, device voltage, and liquid nicotine concentration on electronic cigarette nicotine yield: measurements and model predictions. Nicotine \& Tobacco Research, 17(2), 150-157

Tayyarah, R., \& Long, G. A. (2014). Comparison of select analytes in aerosol from e-cigarettes with smoke from conventional cigarettes and with ambient air.Regulatory Toxicology and Pharmacology, 70(3), 704-710.

Tian, G., Hindle, M., Lee, S., \& Longest, P. W. (2015). Validating CFD Predictions of Pharmaceutical Aerosol Deposition with In Vivo Data.Pharmaceutical Research, 1-18.

Tu, H. H., \& Ray, A. K. (2005). Measurement of activity coefficients from unsteady state evaporation and growth of microdroplets. Chemical Engineering Communications, 192: 474-498.

Turns, S. R. (1996). An Introduction to Combustion (Vol. 287). McGraw-Hill: New York, NY, USA 
Vansickel Andrea, Jeffery Edmiston, Qiwei Liang, Cheryl Duhon, Jianmin Liu, Mohamadi Sarkar. Characterization of Electronic Cigarette Prototype Puff Topography in Adult Exclusive Cigarette Smokers and Adult Exclusive Electronic Cigarette Users. 20 ${ }^{\text {th }}$ Annual Meeting of the Society for Research on Nicotine and Tobacco (SRNT), Seattle, WA, February 5-8, 2014

Zhang, Z., Kleinstreuer, C., Kim, C. S., \& Cheng, Y. S. (2004). Vaporizing microdroplet inhalation, transport, and deposition in a human upper airway model. Aerosol science and technology, 38(1), 36-49.

Zhang, Z., Kleinstreuer, C., Donohue, J. F., \& Kim, C. S. (2005). Comparison of micro-and nano-size particle depositions in a human upper airway model. Journal of aerosol science, 36(2), 211-233.

Zhang, Z., Kleinstreuer, C., \& Kim, C. S. (2006). Transport and uptake of MTBE and ethanol vapors in a human upper airway model. Inhalation Toxicology, 18(3), 169-184.

Zhang Z, Kleinstreuer C (2011) Computational analysis of airflow and nanoparticle deposition in a combined nasal-oral-tracheobronchial airway model. Journal of Aerosol Science, 42(3):174-194

Zhang, Z., Kleinstreuer, C., \& Hyun, S. (2012 a). Size-change and deposition of conventional and composite cigarette smoke particles during inhalation in a subject-specific airway model. Journal of Aerosol Science, 46, 34-52.

Zhang, Z., Kleinstreuer, C., \& Feng, Y. (2012 b). Vapor deposition during cigarette smoke inhalation in a subject-specific human airway model. Journal of Aerosol Science, 53, 40-60 


\section{Table Captions}

Table 1: Initial and boundary conditions for simulating injection of EC droplet-vapor mixture Table 2: Physical properties of vapor species (at $25^{\circ} \mathrm{C}$ and $1 \mathrm{~atm}$ )

Table 3: Scaling factor (SF) values for different inhalation flow rates

Table 4: Best mesh testing for the idealized human upper respiratory system

Table 5: Regional deposition fractions (RDFs) of glycerol, PG, and nicotine after $\mathrm{t}=2.4 \mathrm{~s}$ for EC waveform \#1 (see Fig. 1 for details) 


\section{Figure Captions}

Fig. 1: Realistic EC puff topographies (Vansickel et. al., 2014): (a) Waveform \#1 typical of a conventional cigarette smoker; (b) Waveform \#2 typical of a regular EC user.

Fig. 2: Mixed vapor absorption boundary condition at air-mucus layer: (a) Sketch at the airway wall; (b) Numerical treatment.

Fig. 3: The relationship between scaling sactor SF (i.e., non-dimensionalized single-droplet liquid-vapor interaction intensity) and ratio of droplet number density.

Fig. 4: Configuration and structured hexahedral mesh of idealized human upper respiratory system.

Fig. 5: Mesh-independence test for the idealized human respiratory system: (a) streamlines and velocity contours at cross-sections and the sagittal plane; and (b) velocity profiles at the crosssection B-B' computed with different meshes.

Fig. 6: Model validations for droplet-size change in comparison to experimental data sets: (a) Comparison with Li and Hopke (1993); (b) Comparison with Johnson et al. (2015).

Fig. 7: Model validations for inhaled vapor uptake: (a) Idealized oral cavity geometry with $20 \mathrm{~mm}$ circular mouth inlet; (b) Comparison of regional deposition fractions of MTBE and ethanol vapors with inlet flow rates $\mathrm{Q}_{\mathrm{in}}=15,30$, and $60 \mathrm{~L} / \mathrm{min}$ of $303.15 \mathrm{~K}$.

Fig. 8: Total deposition fractions (TDFs) of different EC-aerosol components with or without liquid-vapor interactions in the idealized human upper airway model $\left(Q_{\text {in }}=27.5 \mathrm{ml} / \mathrm{s}\right.$ at $\mathrm{t}=2$ seconds)

Fig. 9: Airflow field evolution with time for EC-waveform \#1: (a) $t=0.1 \mathrm{~s}$; (b) $\mathrm{t}=0.2 \mathrm{~s}$; (c) $\mathrm{t}=0.4 \mathrm{~s}$; (d) $\mathrm{t}=0.6 \mathrm{~s}$; (e) $\mathrm{t}=0.8 \mathrm{~s} ;$ (f) $\mathrm{t}=1.2 \mathrm{~s}$; and (g) $\mathrm{t}=2.0 \mathrm{~s}$

Fig. 10: Visualization of vortices at $\mathrm{t}=0.4 \mathrm{~s}$ with waveform $\# 1$ when the jet core reaches the first bifurcation - colored by non-dimensionalized helicity $\widetilde{H}$.

Fig. 11: Temperature field evolution with time for $E C$-waveform $\# 1$ : (a) $t=0.1 \mathrm{~s}$; (b) $t=0.2 s$; (c) $\mathrm{t}=0.4 \mathrm{~s} ;(\mathrm{d}) \mathrm{t}=0.8 \mathrm{~s} ;$ (e) $\mathrm{t}=1.2 \mathrm{~s} ;$ (f) $\mathrm{t}=1.6 \mathrm{~s}$; and (g) $\mathrm{t}=2.0 \mathrm{~s}$

Fig. 12: Contours of non-dimensionalized nicotine vapor mass fraction $\tilde{Y}_{s, v}=Y_{s, v} / Y_{s, v}^{(i n)}$ in the sagittal plane $(Z=0)$ of the idealized human upper airway geometry at different times: (a) $t=0.1 \mathrm{~s}$; (b) $\mathrm{t}=0.2 \mathrm{~s}$; (c) $\mathrm{t}=0.3 \mathrm{~s}$; (d) $\mathrm{t}=0.4 \mathrm{~s}$; (e) $\mathrm{t}=0.5 \mathrm{~s}$; (f) $\mathrm{t}=0.6 \mathrm{~s}$; (g) $\mathrm{t}=1.0 \mathrm{~s}$; and (h) $\mathrm{t}=2.4 \mathrm{~s}$

Fig. 13: Contours of non-dimensionalized nicotine vapor mass fraction $\tilde{Y}_{s, v}=Y_{s, v} / Y_{s, v}^{(i n)}$ at different cross sections perpendicular to the centerline of the idealized human upper airway geometry at different times: (a) $\mathrm{t}=0.1 \mathrm{~s}$; (b) $\mathrm{t}=0.2 \mathrm{~s}$; (c) $\mathrm{t}=0.3 \mathrm{~s}$; (d) $\mathrm{t}=0.4 \mathrm{~s}$; (e) $\mathrm{t}=0.5 \mathrm{~s}$; (f) $\mathrm{t}=0.6 \mathrm{~s}$; (g) $\mathrm{t}=1.0 \mathrm{~s} ;$ and $(\mathrm{h}) \mathrm{t}=2.4 \mathrm{~s}$

Fig. 14: Contours of surface density $\left[\mathrm{kg} / \mathrm{m}^{2}\right]$ of nicotine-vapor deposition for EC-waveform \#1 at different times: (a) $\mathrm{t}=0.1 \mathrm{~s}$; (b) $\mathrm{t}=0.2 \mathrm{~s}$; (c) $\mathrm{t}=0.4 \mathrm{~s}$; (d) $\mathrm{t}=0.8 \mathrm{~s}$; (e) $\mathrm{t}=1.2 \mathrm{~s}$; (f) $\mathrm{t}=1.6 \mathrm{~s}$; and (g) $\mathrm{t}=2.4 \mathrm{~s}$

Fig. 15: EC-droplet transport and size change dynamics for waveform \#1 at different times: (a) $\mathrm{t}=0.2 \mathrm{~s}$; (b) $\mathrm{t}=0.4 \mathrm{~s}$; (c) $\mathrm{t}=0.6 \mathrm{~s}$; (d) $\mathrm{t}=0.8 \mathrm{~s}$; (e) $\mathrm{t}=1.0 \mathrm{~s}$; and (f) $\mathrm{t}=1.6 \mathrm{~s}$

Fig. 16: EC-droplet deposition colored by final diameter: (a) for waveform \#1 at $t=2.4 \mathrm{~s}$; and (b) for waveform \#1 followed by one-second holding at $\mathrm{t}=3.4 \mathrm{~s}$. 
Fig. 17: Regional and total deposition fractions of glycerol considering four different steady puffing scenarios: (a) deposition fractions in vapor form; (b) deposition fractions in liquid form; and (c) deposition fractions in both vapor and liquid forms.

Fig. 18: Regional and total deposition fractions of PG considering four different steady puffing scenarios: (a) deposition fractions in vapor form; (b) deposition fractions in liquid form; and (c) deposition fractions in both vapor and liquid forms.

Fig. 19: Regional and total deposition fractions of nicotine considering four different steady puffing scenarios: (a) deposition fractions in vapor form; (b) deposition fractions in liquid form; and (c) deposition fractions in both vapor and liquid forms.

Fig. 20: Regional and total deposition fractions of nicotine for different transient EC-smoke inhalation conditions (i.e., waveform \#1, waveform \#1 with one-second holding, and waveform \#2): (a) deposition fractions in vapor form; (b) deposition fractions in liquid form; and (c) deposition fractions in both vapor and liquid forms.

Fig. 21: Comparisons of key aspects of droplet-vapor dynamics for different puffing scenarios: (a) percentage-changes in liquid-vapor mass transfer; and (b) averaged droplet-size growth ratio. 
Table 1: Initial and boundary conditions for simulating injection of EC droplet-vapor mixture

\begin{tabular}{|c|c|c|c|c|}
\hline Droplet initial diameter & \multicolumn{4}{|c|}{$400 \mathrm{~nm}$} \\
\hline Initial droplet density & \multicolumn{4}{|c|}{$1106.70 \mathrm{~kg} / \mathrm{m}^{3}$} \\
\hline Inlet air flow rate & \multicolumn{4}{|c|}{ From $12 \mathrm{ml} / \mathrm{s}$ to $27.5 \mathrm{ml} / \mathrm{s}$} \\
\hline $\begin{array}{l}\text { Total e-cigarette aerosol mass } \\
\text { flow rate }\end{array}$ & \multicolumn{4}{|c|}{$4.17 \mathrm{mg} / \mathrm{s}$ for $27.5 \mathrm{ml} / \mathrm{s}$} \\
\hline Inlet temperature & \multicolumn{4}{|c|}{$360.15 \mathrm{~K}$} \\
\hline Inlet relative humidity & \multicolumn{4}{|c|}{$50.00 \%$} \\
\hline \multirow{2}{*}{$\begin{array}{l}\text { Vapor-liquid mass fraction } \\
\text { partitions }(w t / w t)\end{array}$} & Water & Glycerol & PG & Nicotine \\
\hline & N/A & $0.1293 \%$ & $18.20 \%$ & $5.09 \%$ \\
\hline Initial air-vapor mixture density & \multicolumn{4}{|c|}{$1.2842 \mathrm{~kg} / \mathrm{m}^{3}$} \\
\hline \multirow{2}{*}{$\begin{array}{c}\text { Initial vapor species mass } \\
\text { fraction }\end{array}$} & Water & Glycerol & PG & Nicotine \\
\hline & $1.98 \%$ & $0.0048 \%$ & $0.75 \%$ & $0.013 \%$ \\
\hline \multirow{2}{*}{$\begin{array}{l}\text { Initial droplet component mass } \\
\text { fraction }\end{array}$} & Water & Glycerol & PG & Nicotine \\
\hline & $15 \%$ & $38.96 \%$ & $43.34 \%$ & $2.7 \%$ \\
\hline
\end{tabular}


Table 2: Physical properties of vapor species (at $25^{\circ} \mathrm{C}$ and $1 \mathrm{~atm}$ )

\begin{tabular}{|l|l|l|l|l|}
\hline & \multicolumn{1}{|c|}{$\tilde{D}_{a-v, s}\left[\mathrm{~cm}^{2} / \mathrm{s}\right]$} & \multicolumn{1}{|c|}{$\tilde{D}_{m, s}\left[\mathrm{~cm}^{2} / \mathrm{s}\right]$} & \multicolumn{1}{c|}{$k_{H, s, a-m}$} & $\Gamma_{s, w}\left[\mathrm{~cm}^{-1}\right]$ \\
\hline Glycerol & 0.0877 & $9.30 \mathrm{e}-6^{\mathrm{b}}$ & $3.93 \mathrm{e}-9^{\mathrm{e}}$ & $2.70 \mathrm{e}+7$ \\
\hline PG & 0.1060 & $1.23 \mathrm{e}-5^{\mathrm{c}}$ & $4.90 \mathrm{e}-7^{\mathrm{f}}$ & $2.39 \mathrm{e}+5$ \\
\hline Nicotine & $0.0650^{\mathrm{a}}$ & $8.60 \mathrm{e}-6^{\mathrm{d}}$ (in epithelium) & $3.31 \mathrm{e}-7^{\mathrm{g}}$ & $4.00 \mathrm{e}+5$ \\
\hline
\end{tabular}

Notes:

(1) Values of $\tilde{D}_{m, s}$ are approximated by the values in water unless otherwise noted.

(2) The dimensionless gas-liquid Henry's law constants $k_{H, s, a-m}$ are assumed for air-water interface unless otherwise noted. Same assumptions and reasons have been discussed in Keyhani et al. (1997). Conversions between different definitions of Henry's law constant provided by http://www.mpch-mainz.mpg.de/ sander/res/henry.html.

Data Sources:

${ }^{a}$ Eatough et al. (1989)

${ }^{\mathrm{b}}$ Hayduk and Laudie (1974)

${ }^{\mathrm{c}}$ http://www.gsi-net.com/en/publications/gsi-chemical-database/single/469.html

${ }^{\mathrm{d}}$ Gowadia and Dunn-Rankin (2010)

${ }^{\mathrm{e}} \mathrm{http} / / / \mathrm{www}$. inchem.org/documents/sids/sids/56815.pdf

$\mathrm{f}$ http://www.atsdr.cdc.gov/toxprofiles/tp189-c3.pdf

${ }_{\mathrm{g}}^{\mathrm{http}}$ //tools.niehs.nih.gov/cebs3/ntpviews/index.cfm?action=testarticle.properties\&cas number $=54-11-5$ 
Table 3: Scaling factor (SF) values for different inhalation flow rates

\begin{tabular}{|c|c|c|c|}
\hline Q & Total Puff Volume $[\mathbf{m l}]$ & $\mathbf{Q}_{\mathbf{N}, \text { real }} / \mathbf{Q}_{\mathbf{N}, \mathbf{s i n}}$ & $\mathbf{S F}$ \\
\hline $12 \mathrm{ml} / \mathrm{s}$ (for 2 seconds) & $24 \mathrm{ml}$ & $0.6634 \mathrm{e}+7$ & $6.8022 \mathrm{e}-4$ \\
\hline $20 \mathrm{ml} / \mathrm{s}$ (for 2 seconds) & $40 \mathrm{ml}$ & $1.1057 \mathrm{e}+7$ & $5.3659 \mathrm{e}-4$ \\
\hline $27.5 \mathrm{ml} / \mathrm{s}$ (for 2 seconds) & $55 \mathrm{ml}$ & $1.5203 \mathrm{e}+7$ & $4.6284 \mathrm{e}-4$ \\
\hline $18.3 \mathrm{ml} / \mathrm{s}$ (for 3 seconds) & $55 \mathrm{ml}$ & $1.0117 \mathrm{e}+7$ & $5.5918 \mathrm{e}-4$ \\
\hline $\begin{array}{c}\text { Transient Waveform \#1 26.6 ml/s } \\
\text { (for 2.4 seconds) }\end{array}$ & $63.9 \mathrm{ml}$ & $1.4705 \mathrm{e}+7$ & $4.7005 \mathrm{e}-4$ \\
\hline $\begin{array}{c}\text { Transient Waveform \#2 12.78 ml/s } \\
\text { (for 5.0 seconds) }\end{array}$ & $63.9 \mathrm{ml}$ & $0.7065 \mathrm{e}+7$ & $6.6063 \mathrm{e}-4$ \\
\hline
\end{tabular}


Table 4: Best mesh testing for the idealized human upper respiratory system

\begin{tabular}{|c|c|c|c|}
\hline Mesh No. & $\begin{array}{c}\text { Total Number of } \\
\text { Elements }\end{array}$ & $\begin{array}{c}\text { Total Number of } \\
\text { Nodes }\end{array}$ & $\begin{array}{c}\text { Refinement Factor at } \mathbf{x}, \mathbf{y}, \text { or } \mathbf{z} \\
\text { directions }\end{array}$ \\
\hline $\mathbf{1}$ & 352,901 & 337,744 & N/A \\
\hline $\mathbf{2}$ (Final) & $2,374,679$ & $2,319,768$ & 2.0 \\
\hline $\mathbf{3}$ & $19,755,522$ & $19,528,440$ & 2.0 \\
\hline
\end{tabular}


Table 5: Regional deposition fractions (RDFs) of glycerol, PG, and nicotine after $\mathrm{t}=2.4 \mathrm{~s}$ for EC waveform \#1 (see Fig. 1 for details)

\begin{tabular}{|c|c|c|c|c|c|c|c|}
\hline \multirow{2}{*}{\multicolumn{2}{|c|}{$\begin{array}{c}\text { Accumulated inflow } \\
\text { mass at } t=2.4 \mathrm{~s}\end{array}$}} & \multicolumn{2}{|c|}{ Glycerol } & \multicolumn{2}{|c|}{ PG } & \multicolumn{2}{|c|}{ Nicotine } \\
\hline & & \multicolumn{2}{|c|}{$2.5041 \mathrm{mg}$} & \multicolumn{2}{|c|}{$3.8173 \mathrm{mg}$} & \multicolumn{2}{|c|}{$0.2116 \mathrm{mg}$} \\
\hline \multicolumn{2}{|c|}{ Phase } & Vapor & Liquid & Vapor & Liquid & Vapor & Liquid \\
\hline \multirow{4}{*}{ RDFs } & Oral Cavity & $0.0391 \%$ & $5.2934 \%$ & $5.3577 \%$ & $4.6288 \%$ & $1.3482 \%$ & $5.0951 \%$ \\
\hline & Oropharynx & $0.0070 \%$ & $2.5045 \%$ & $1.0879 \%$ & $2.2151 \%$ & $0.3057 \%$ & $2.4266 \%$ \\
\hline & Trachea & $0.0010 \%$ & $0.4526 \%$ & $0.2648 \%$ & $0.4252 \%$ & $0.0559 \%$ & $0.4476 \%$ \\
\hline & B1 to B3 & $0.0009 \%$ & $2.2254 \%$ & $0.4925 \%$ & $2.0613 \%$ & $0.0772 \%$ & $2.1940 \%$ \\
\hline \multicolumn{2}{|r|}{ TDF } & $0.0480 \%$ & $10.5239 \%$ & $7.2029 \%$ & $9.3304 \%$ & $1.7869 \%$ & $10.1632 \%$ \\
\hline
\end{tabular}



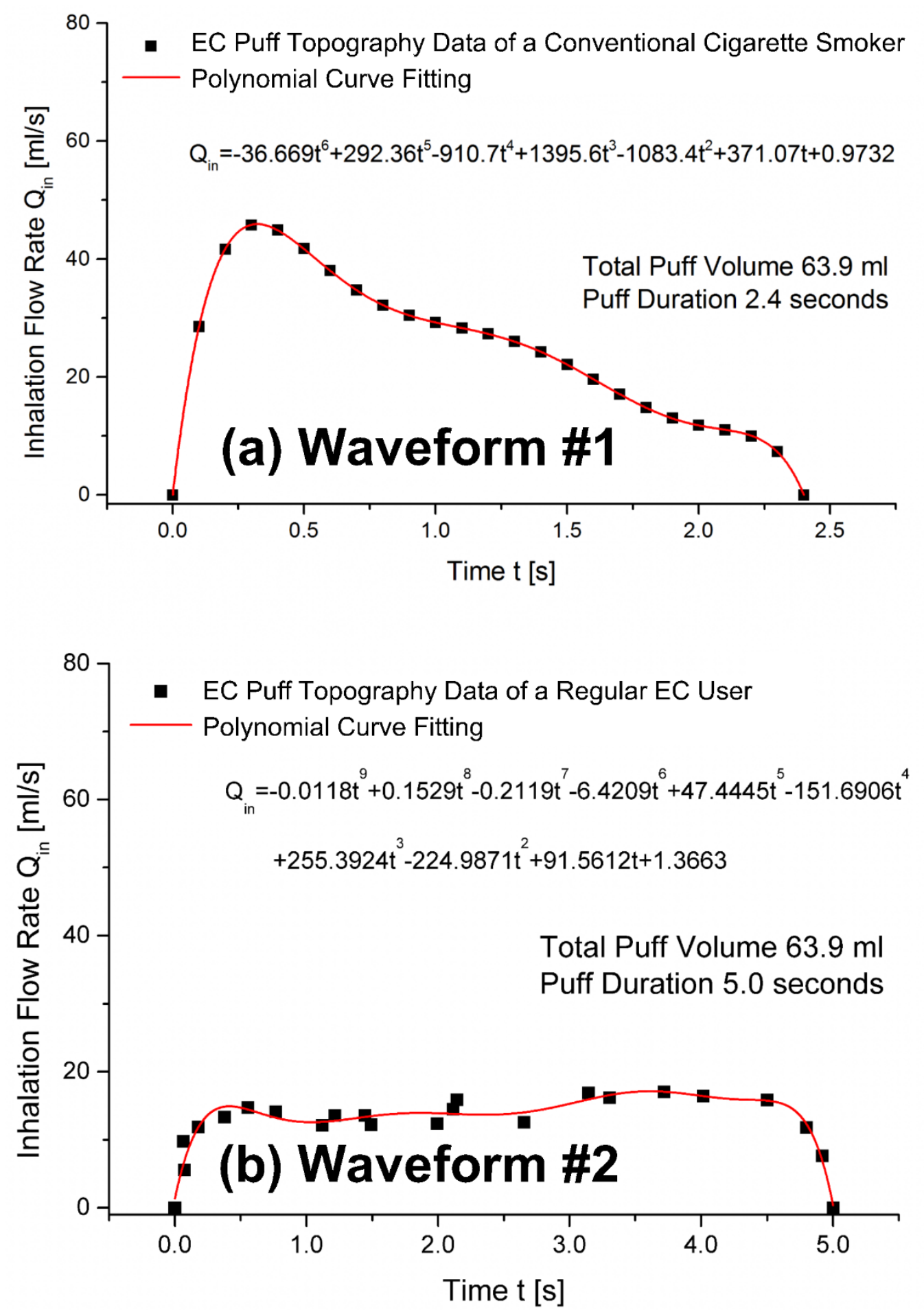

Fig. 1: Realistic EC puff topographies (Vansickel et. al., 2014): (a) Waveform \#1 typical of a conventional cigarette smoker; (b) Waveform \#2 typical of a regular EC user. 


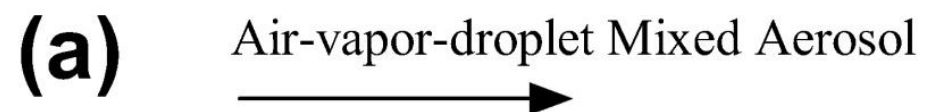

(1) Satisfy Henry's Law: $Y_{s, v l_{n}=0}=k_{H, s, a-m} Y_{s, v l_{n+=0}}$

(2) Satisfy Diffusion Flux Equality at wall $n=0$

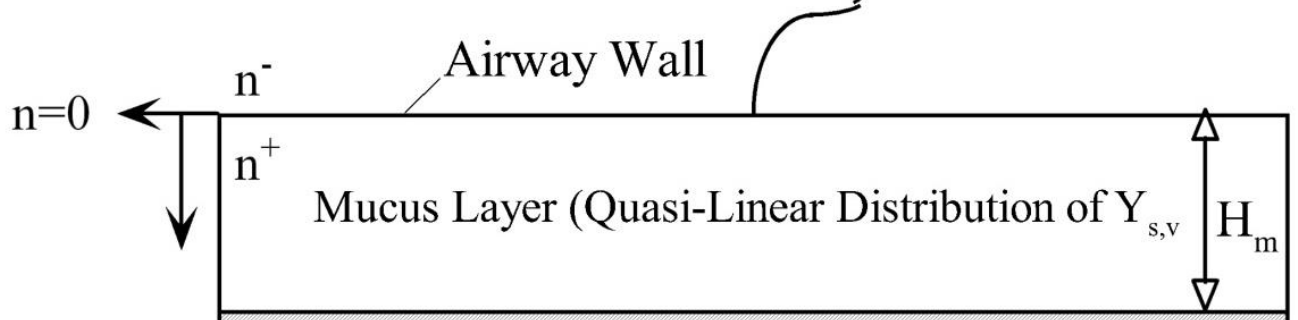

(3) Satisfy $Y_{s, v}=0$ in Tissue Compartment

Tissue (Rapid Removal of Vapor Molecules)

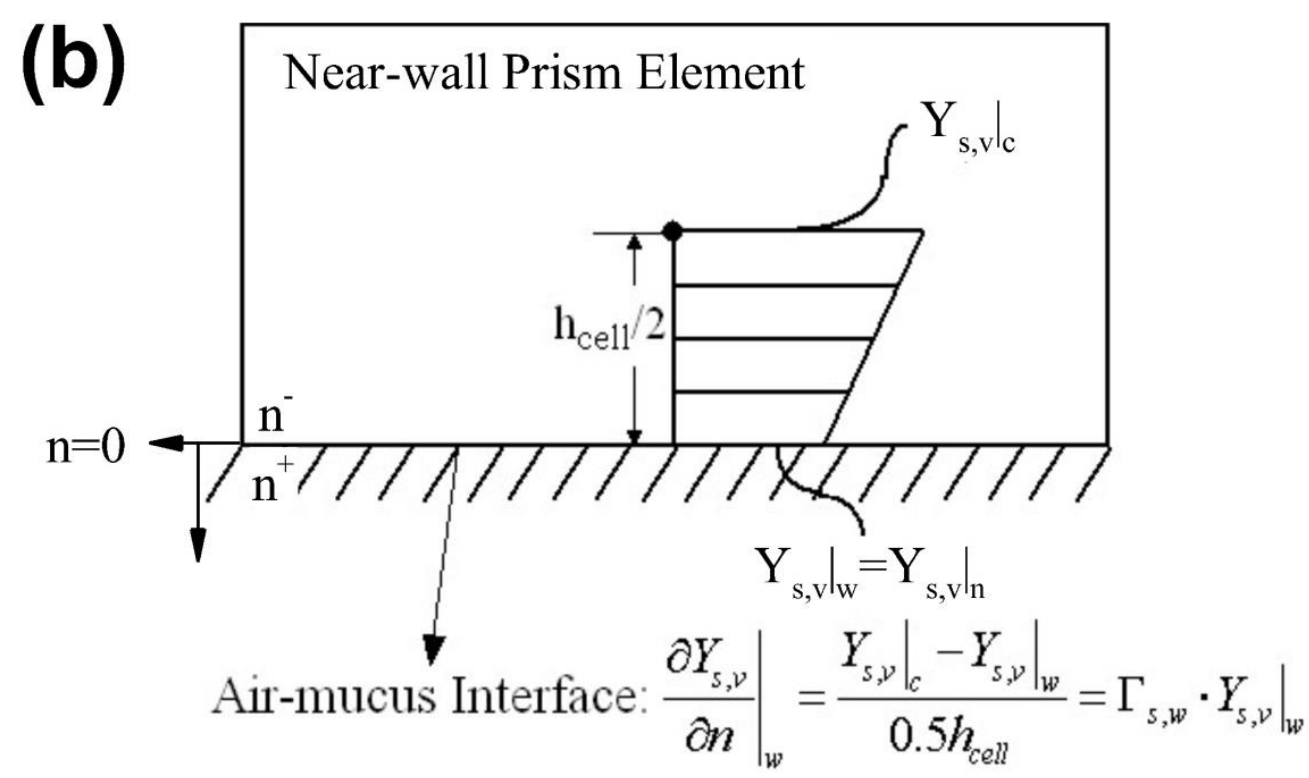

Fig. 2: Mixed vapor absorption boundary condition at air-mucus layer: (a) Sketch at the airway wall; (b) Numerical treatment. 


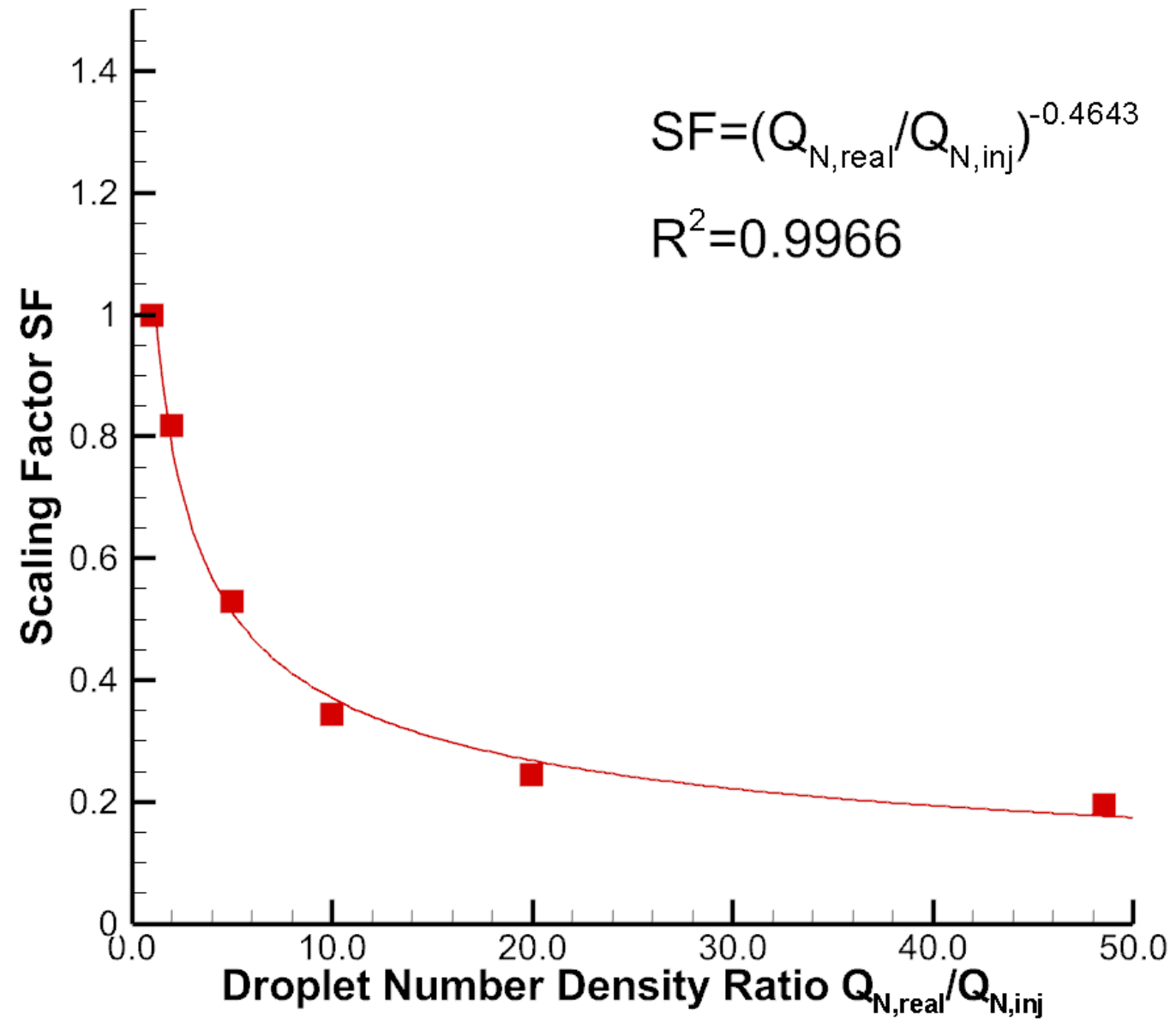

Fig. 3: The relationship between scaling factor SF (i.e., non-dimensionalized single-droplet liquid-vapor interaction intensity) and ratio of droplet number density. 


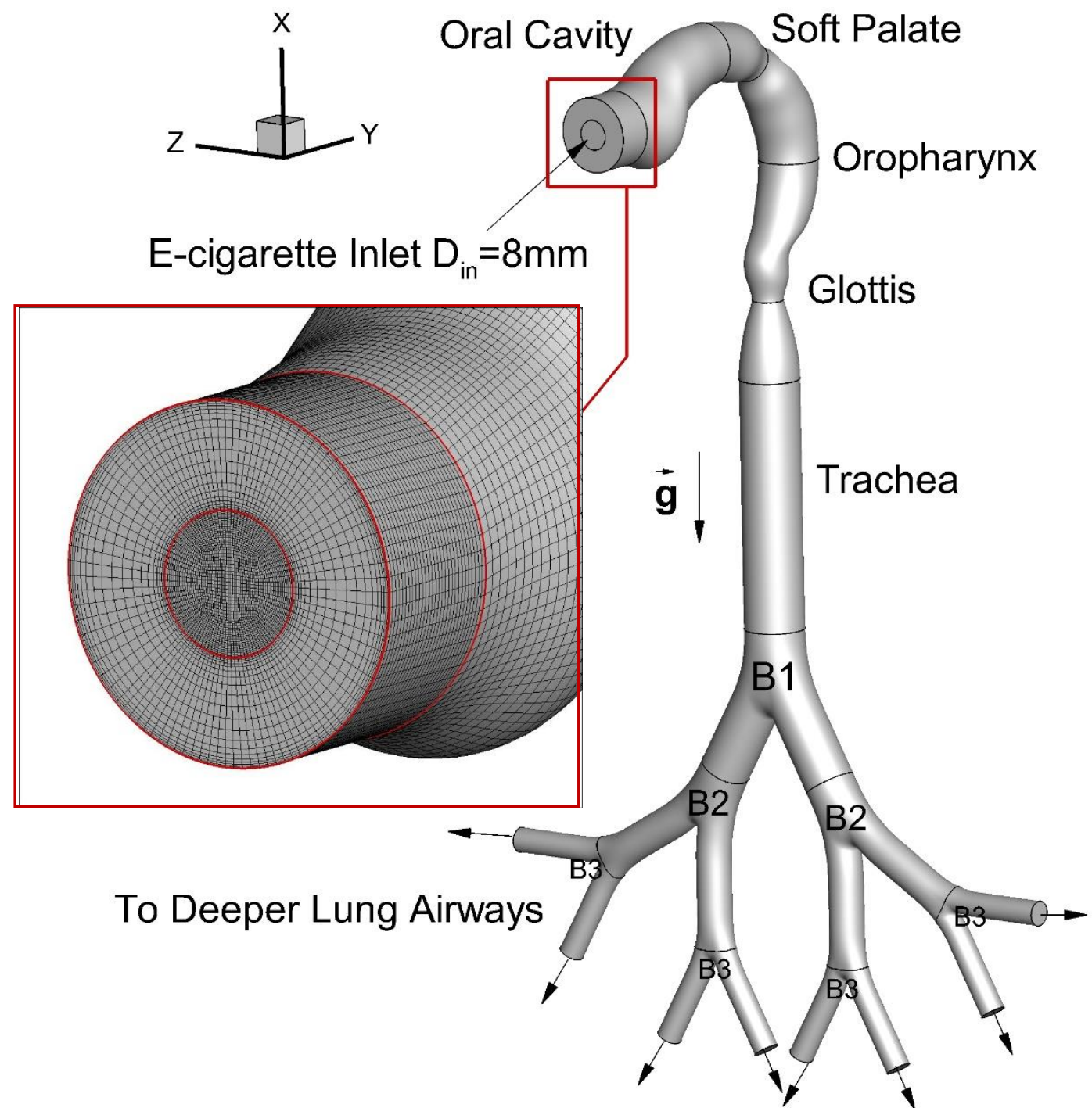

Fig. 4: Configuration and structured hexahedral mesh of idealized human upper respiratory system 


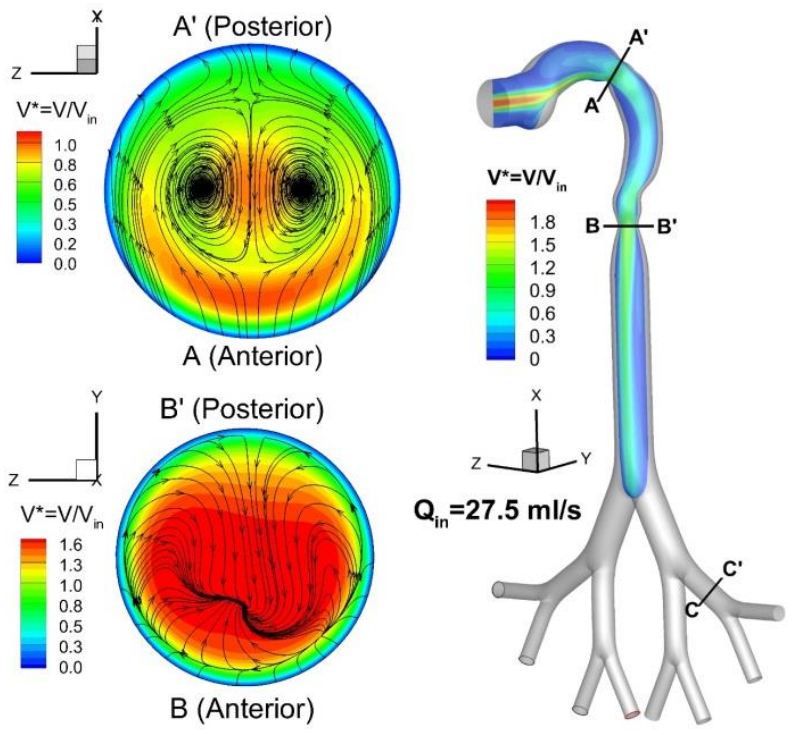

(a)

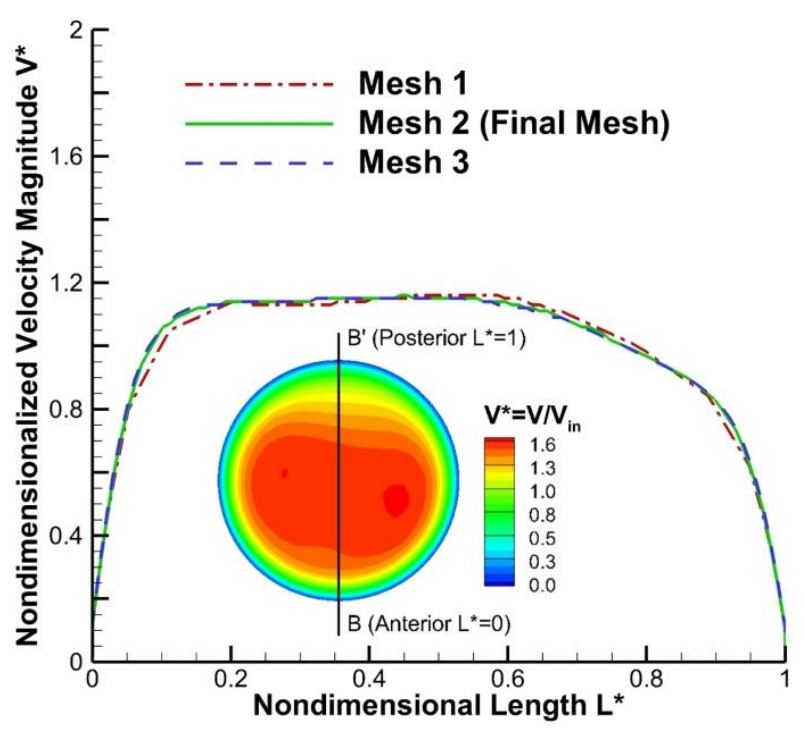

(b)

Fig. 5: Mesh-independence test for the idealized human respiratory system: (a) streamlines and velocity contours at cross-sections and the sagittal plane; and (b) velocity profiles at the crosssection B-B' computed with different meshes. 

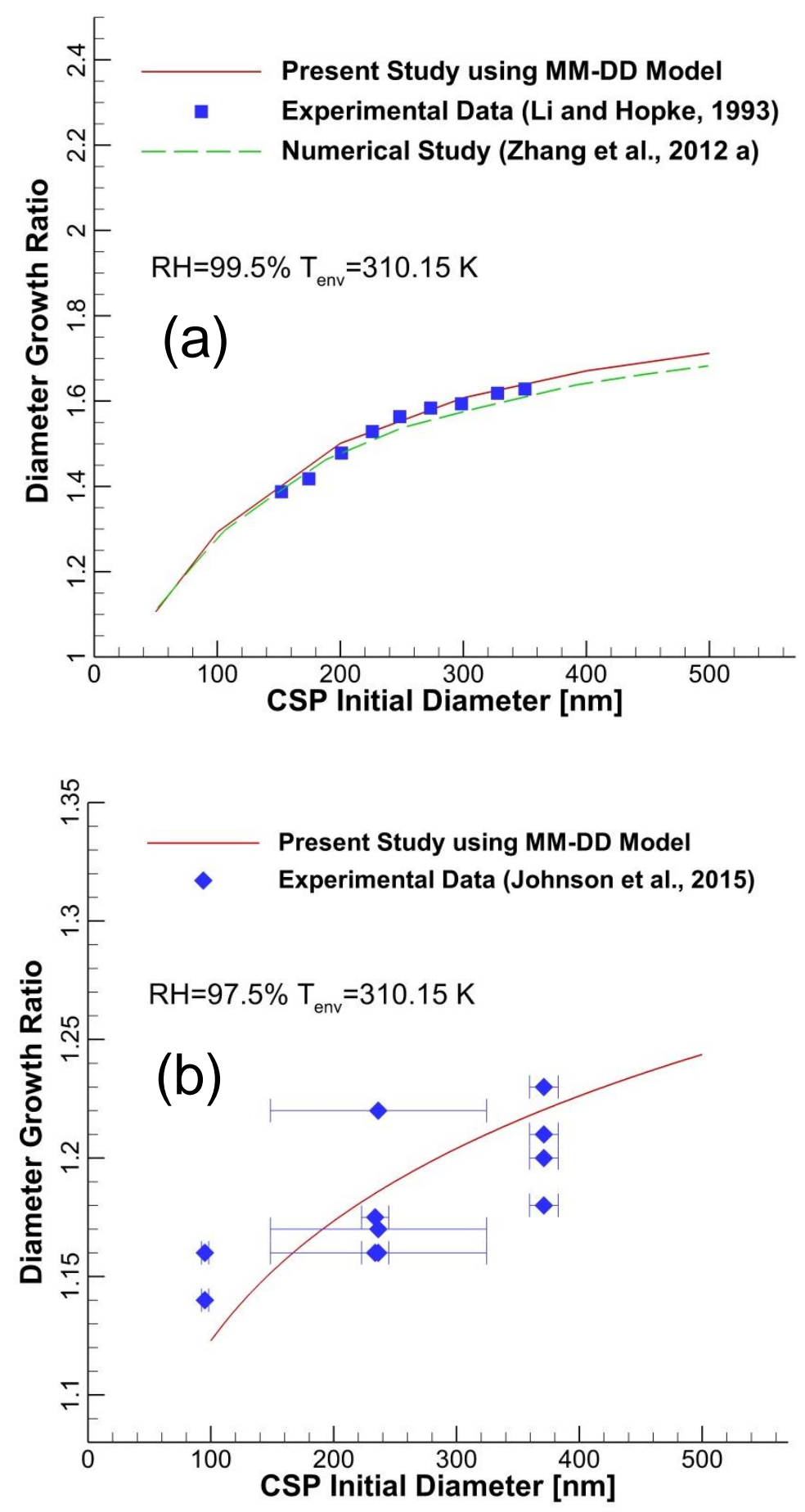

Fig. 6: Model validations for droplet-size change in comparison to experimental data sets: (a) Comparison with Li and Hopke (1993); (b) Comparison with Johnson et al. (2015) 

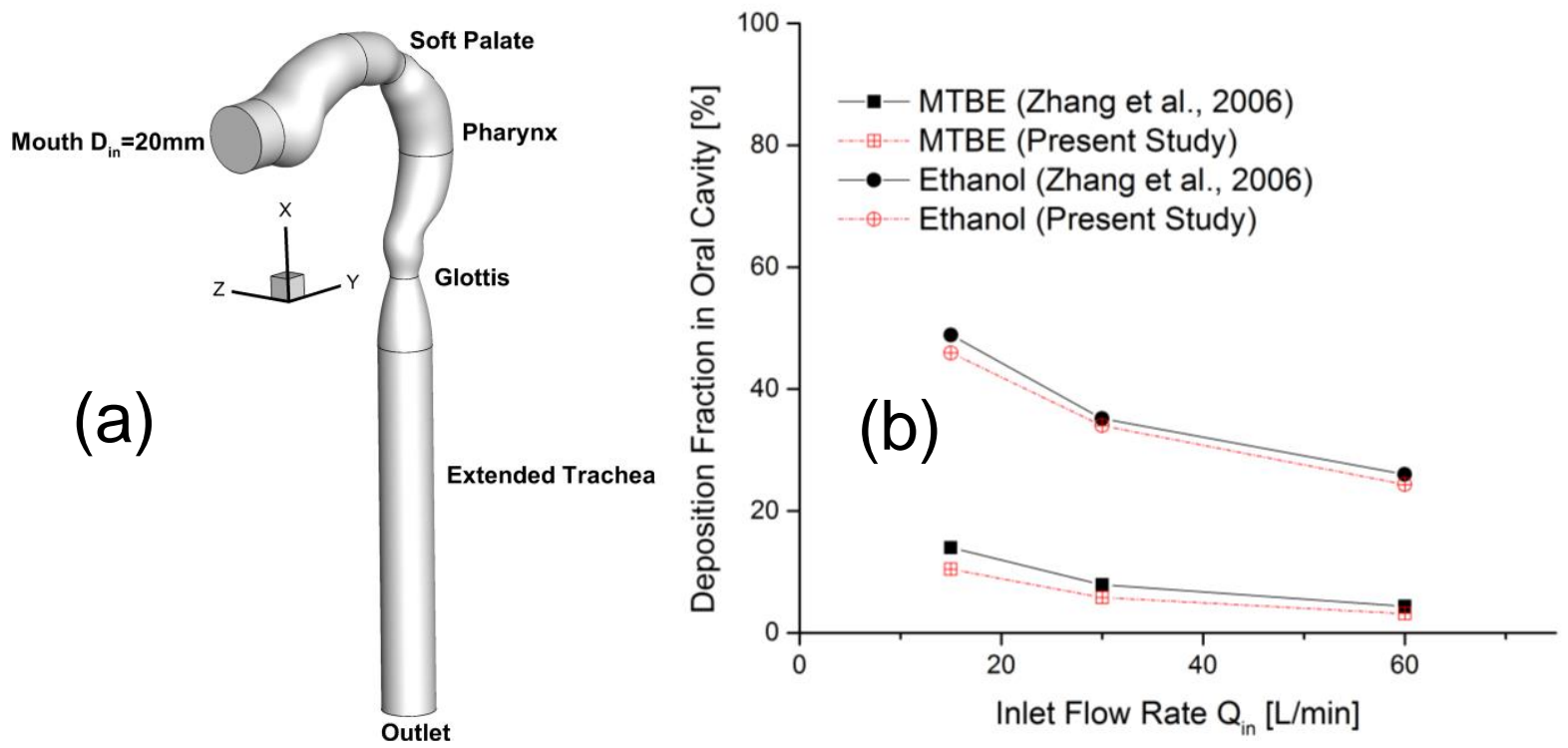

Fig. 7: Model validations for inhaled vapor uptake: (a) Idealized oral cavity geometry with $20 \mathrm{~mm}$ circular mouth inlet; (b) Comparison of regional deposition fractions of MTBE and ethanol vapors with inlet flow rates $Q_{i n}=15,30$, and $60 \mathrm{~L} / \mathrm{min}$ of $303.15 \mathrm{~K}$. 


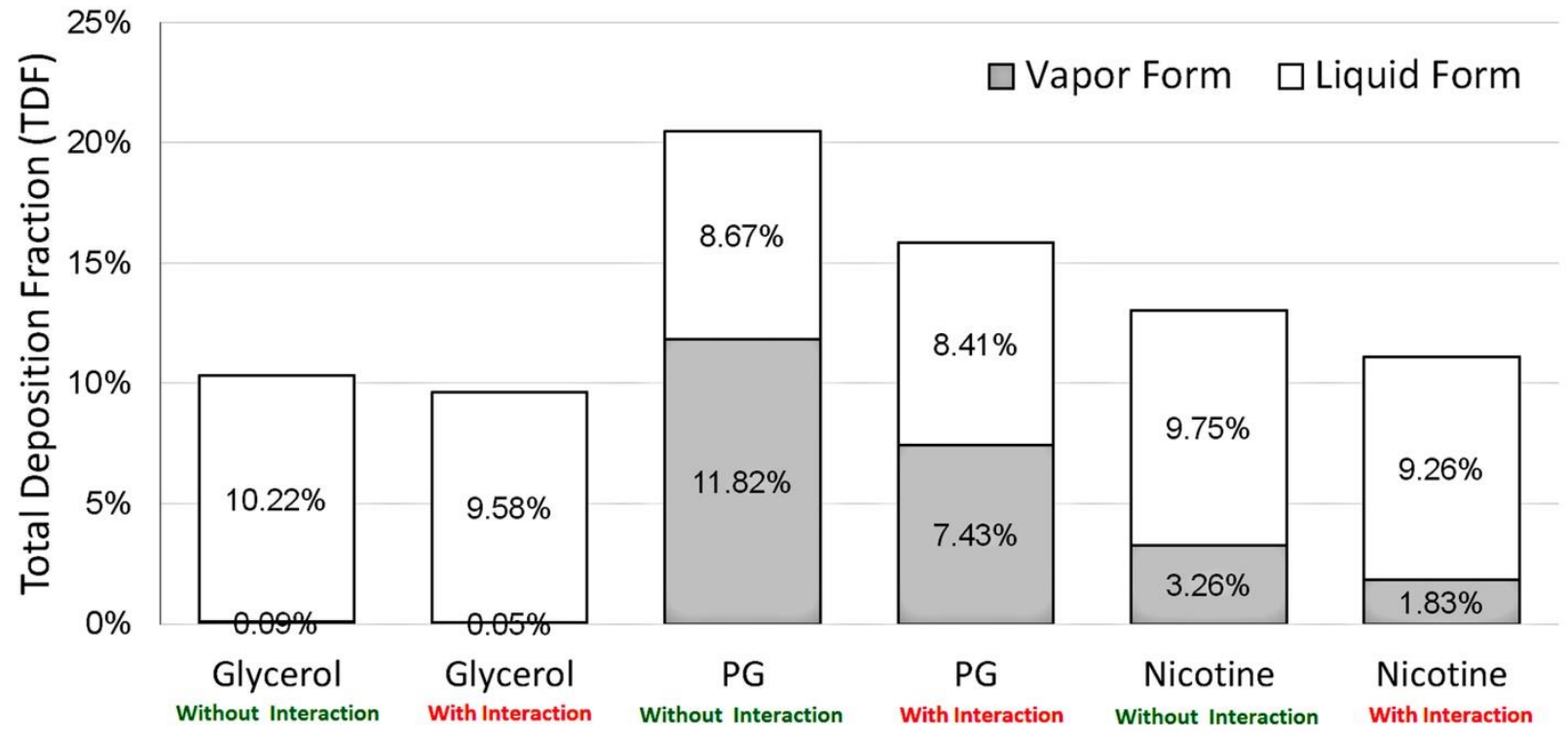

Fig. 8: Total deposition fractions (TDFs) of different EC-aerosol components with or without liquid-vapor interactions in the idealized human upper airway model ( $Q_{i n}=27.5 \mathrm{ml} / \mathrm{s}$ at $\mathrm{t}=2$ seconds) 

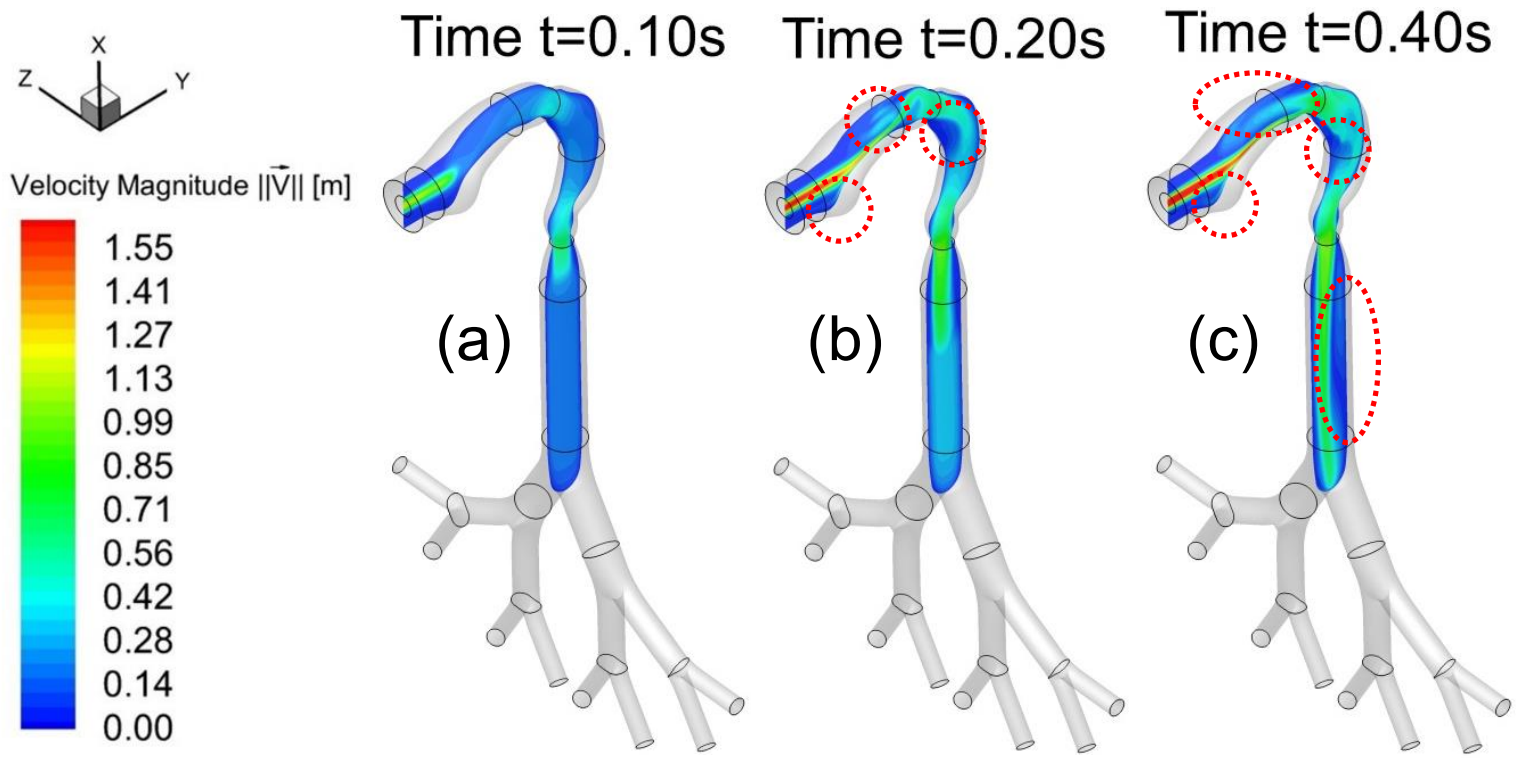

\section{Time $\mathrm{t}=0.60 \mathrm{~s}$ Time $\mathrm{t}=0.80 \mathrm{~s}$ Time $\mathrm{t}=1.20 \mathrm{~s}$ Time $\mathrm{t}=2.00 \mathrm{~s}$}
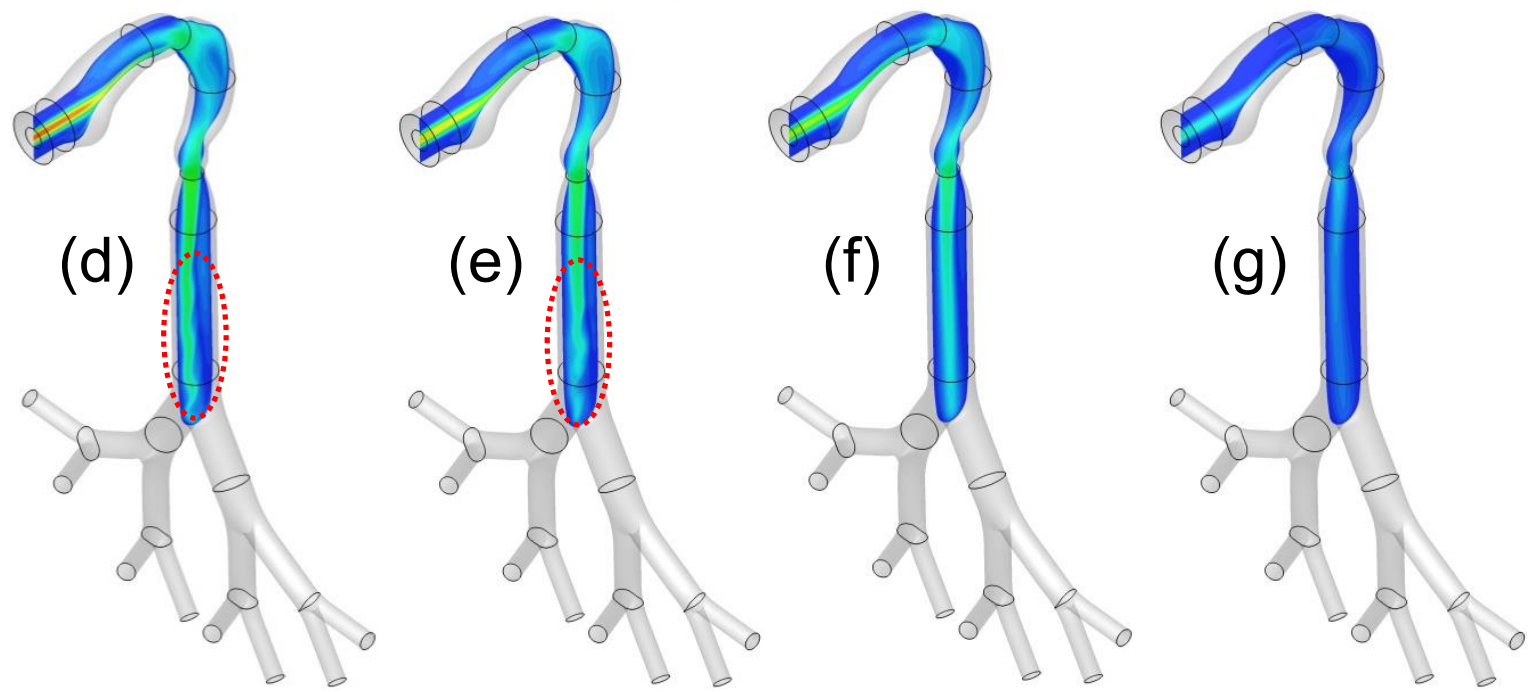

Fig. 9: Airflow field evolution with time for EC-waveform \#1: (a) $t=0.1 \mathrm{~s}$; (b) $t=0.2 s$; (c) $t=0.4 s$; (d) $\mathrm{t}=0.6 \mathrm{~s}$; (e) $\mathrm{t}=0.8 \mathrm{~s}$; (f) $\mathrm{t}=1.2 \mathrm{~s}$; and (g) $\mathrm{t}=2.0 \mathrm{~s}$ 

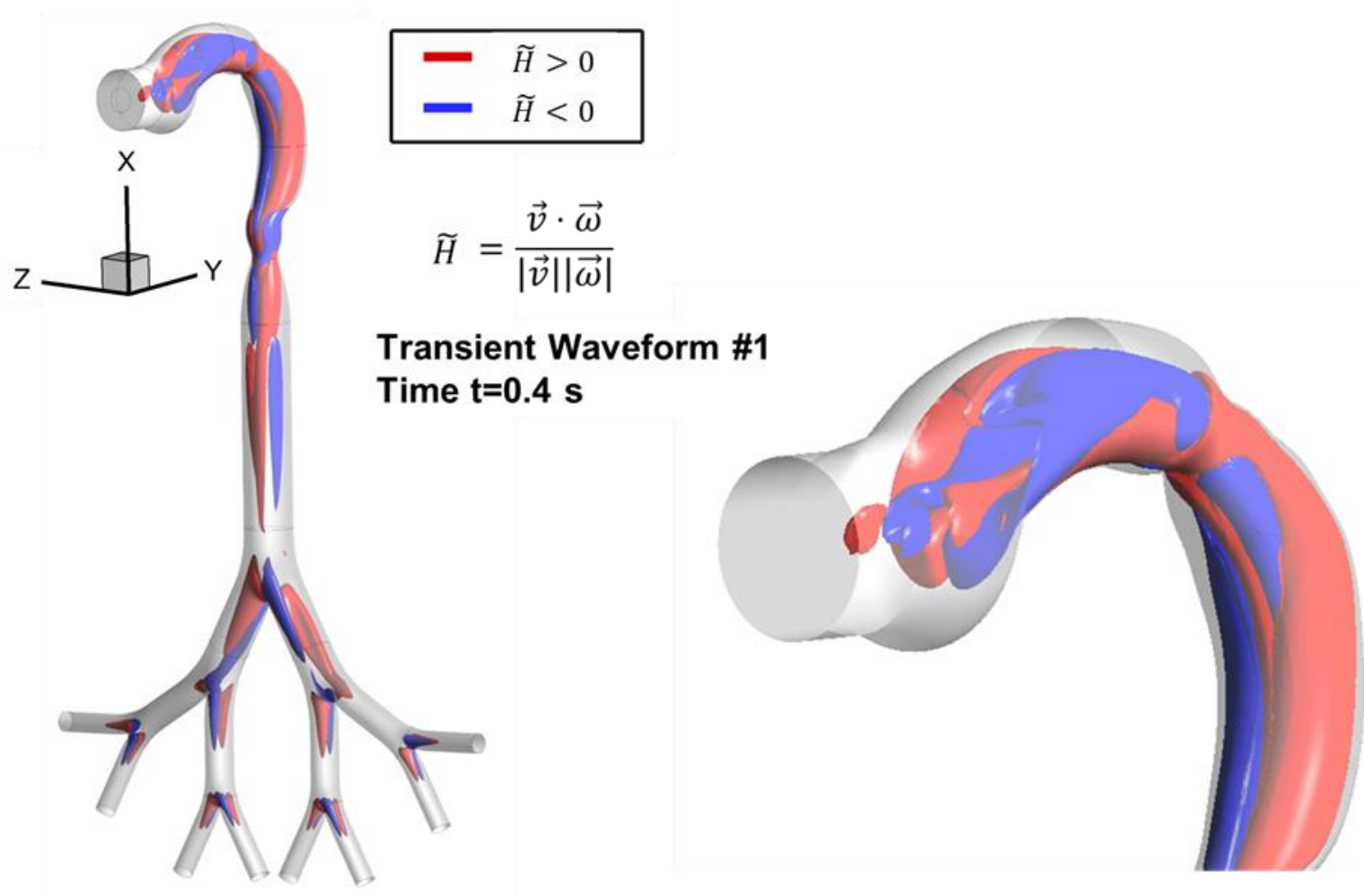

Fig. 10: Visualization of vortices at $\mathrm{t}=0.4 \mathrm{~s}$ with waveform $\# 1$ when the jet core reaches the first bifurcation - colored by non-dimensionalized helicity $\widetilde{H}$. 


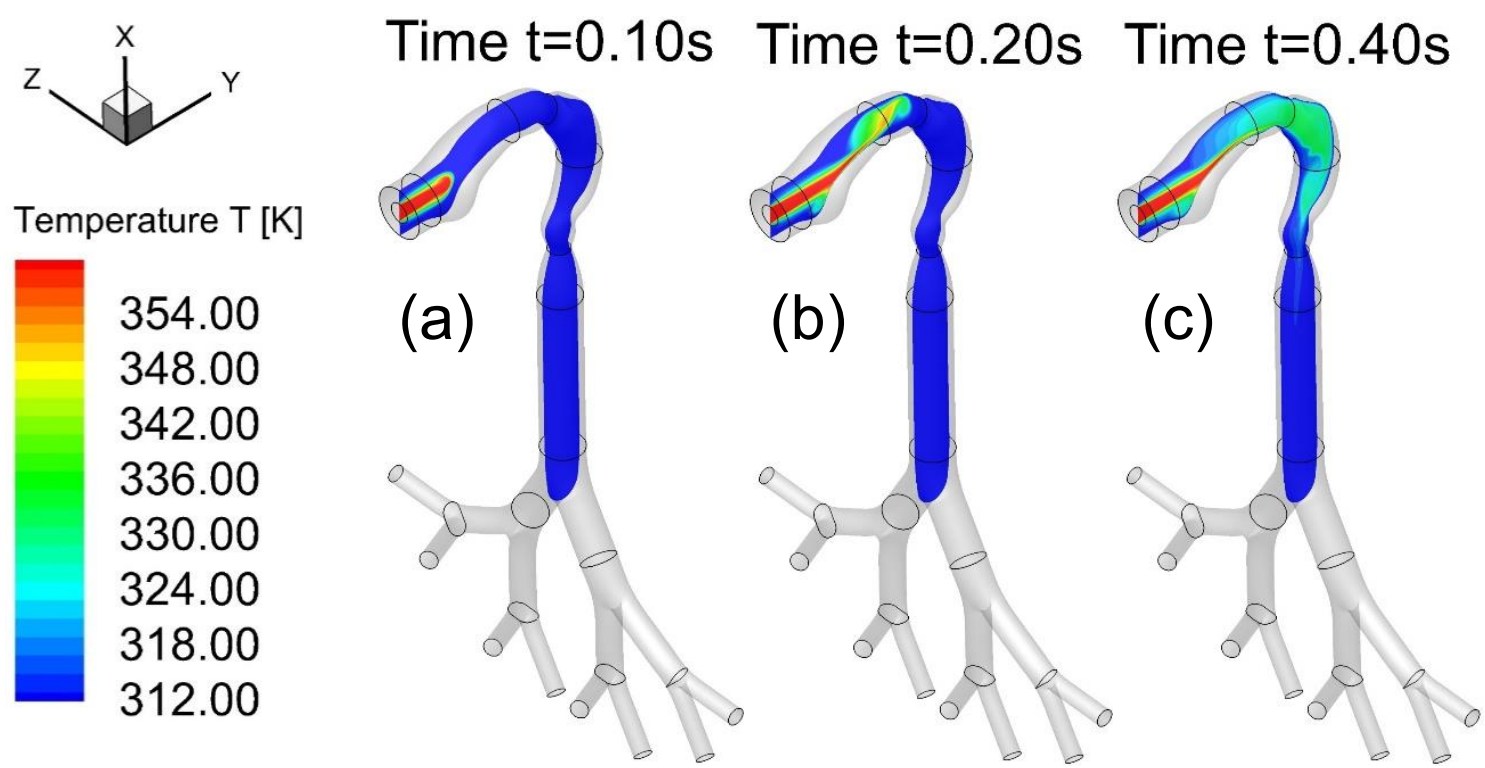

Time $t=0.80 \mathrm{~s}$ Time $t=1.20 \mathrm{~s}$ Time $t=1.60 \mathrm{~s}$ Time $\mathrm{t}=2.00 \mathrm{~s}$
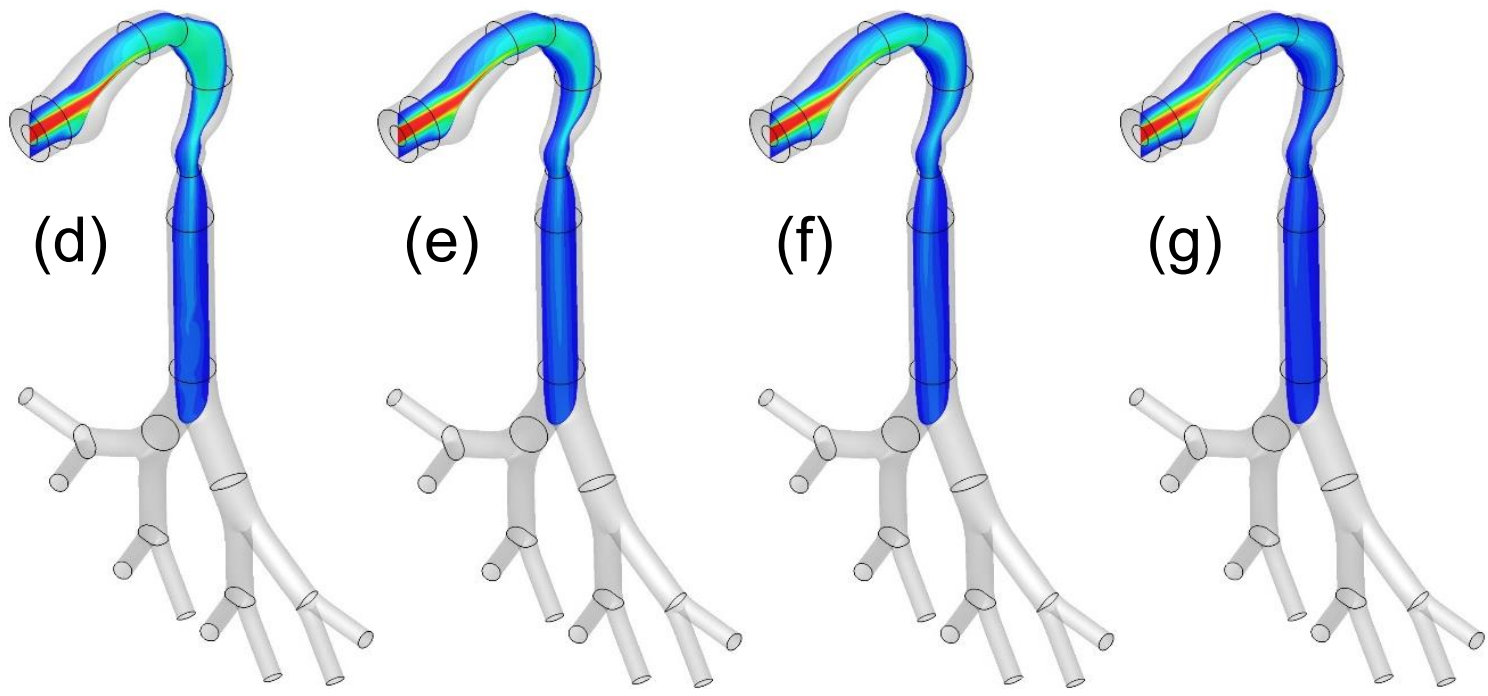

Fig. 11: Temperature field evolution with time for EC-waveform \#1: (a) $t=0.1 \mathrm{~s}$; (b) $t=0.2 \mathrm{~s}$; (c) $\mathrm{t}=0.4 \mathrm{~s}$; (d) $\mathrm{t}=0.8 \mathrm{~s}$; (e) $\mathrm{t}=1.2 \mathrm{~s}$; (f) $\mathrm{t}=1.6 \mathrm{~s}$; and (g) $\mathrm{t}=2.0 \mathrm{~s}$ 

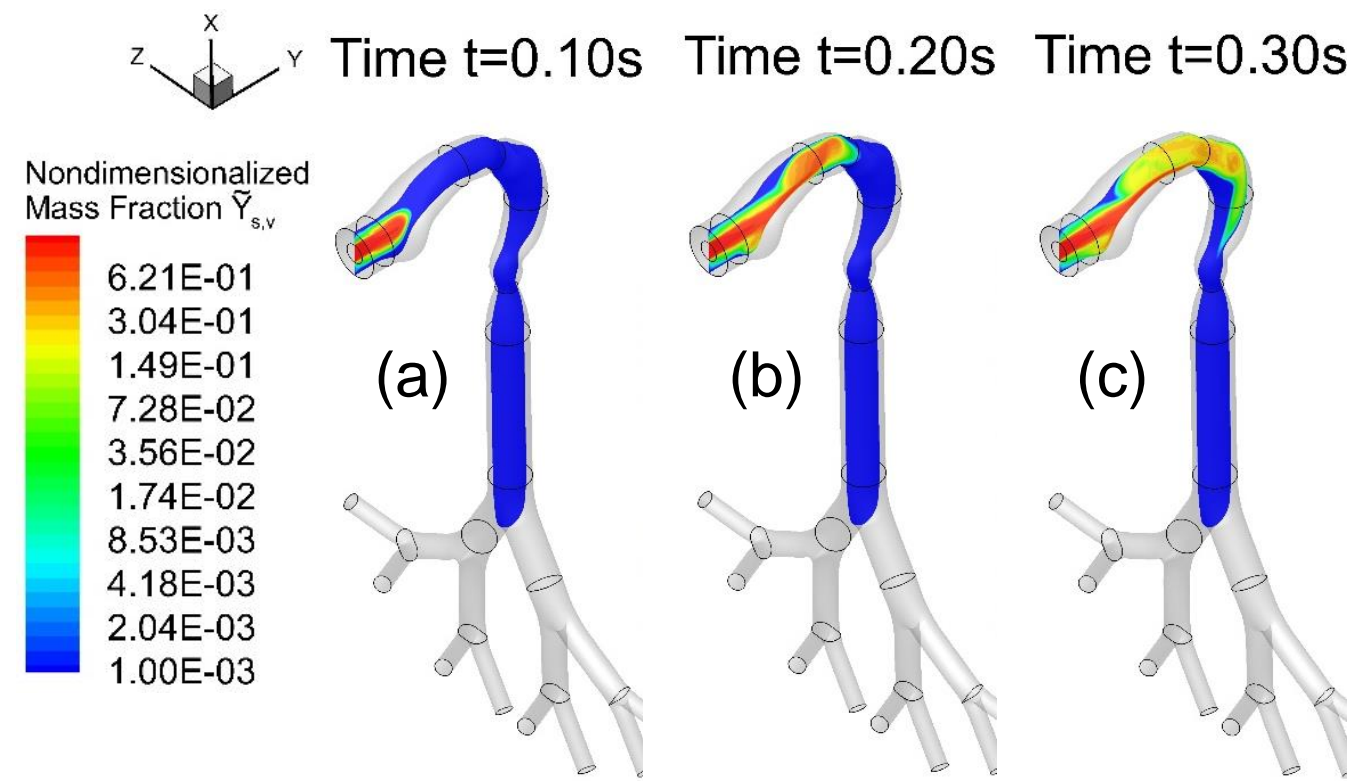

Time $t=0.40 \mathrm{~s}$

\section{Time $\mathrm{t}=0.50 \mathrm{~s}$}
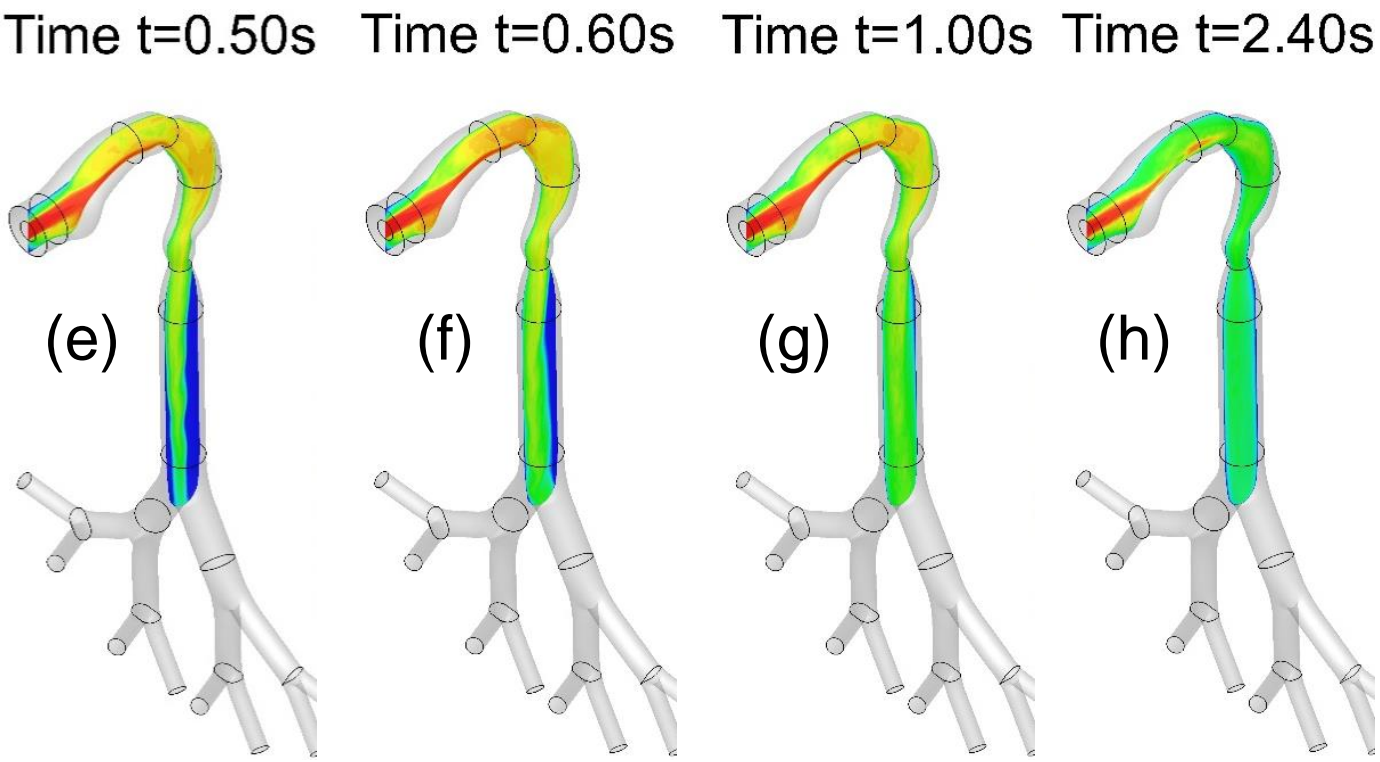

Fig. 12: Contours of non-dimensionalized nicotine vapor mass fraction $\tilde{Y}_{s, v}=Y_{s, v} / Y_{s, v}^{(i n)}$ in the sagittal plane $(Z=0)$ of the idealized human upper airway geometry at different times: (a) $t=0.1 \mathrm{~s}$; (b) $\mathrm{t}=0.2 \mathrm{~s}$; (c) $\mathrm{t}=0.3 \mathrm{~s}$; (d) $\mathrm{t}=0.4 \mathrm{~s}$; (e) $\mathrm{t}=0.5 \mathrm{~s}$; (f) $\mathrm{t}=0.6 \mathrm{~s}$; (g) $\mathrm{t}=1.0 \mathrm{~s}$; and (h) $\mathrm{t}=2.4 \mathrm{~s}$ 


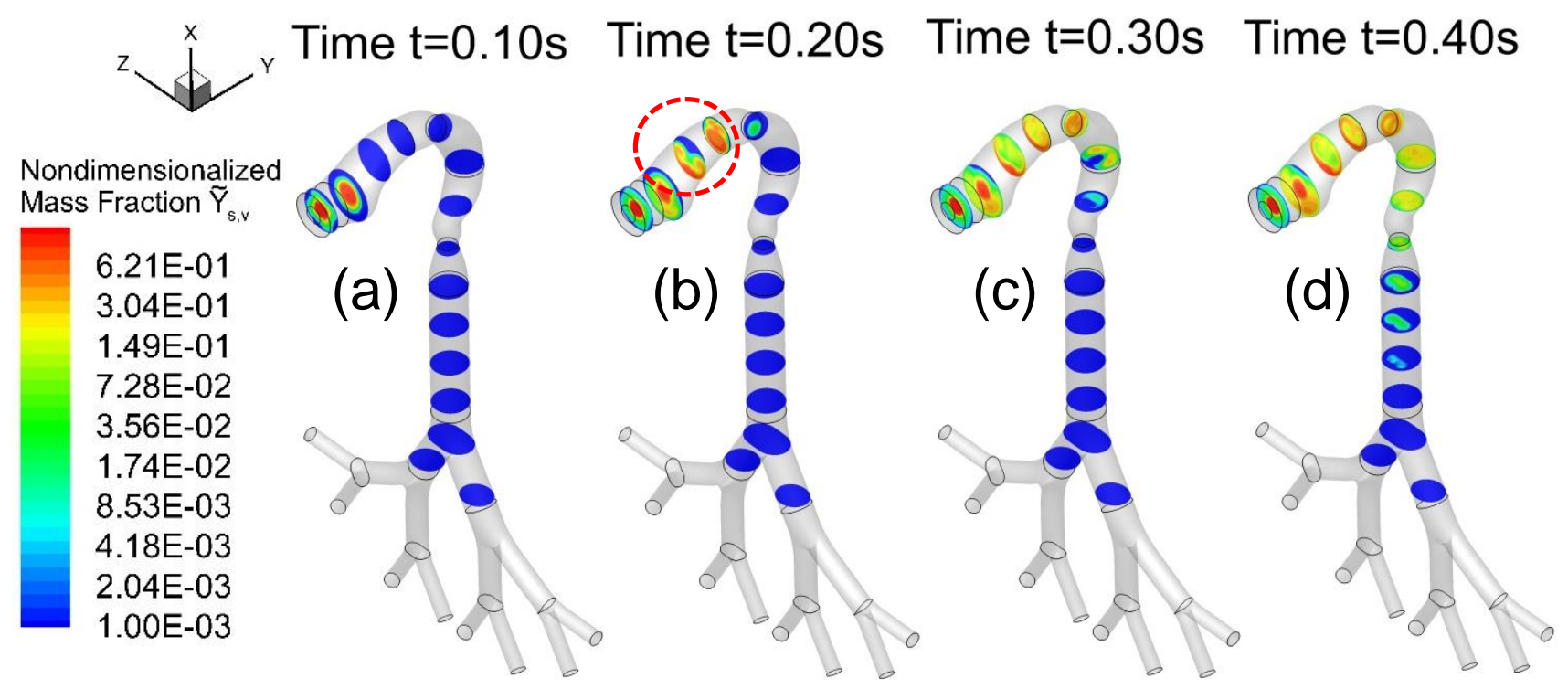

\section{Time $\mathrm{t}=0.50 \mathrm{~s}$ Time $\mathrm{t}=0.60 \mathrm{~s}$ Time $\mathrm{t}=1.00 \mathrm{~s}$ Time $\mathrm{t}=2.40 \mathrm{~s}$}
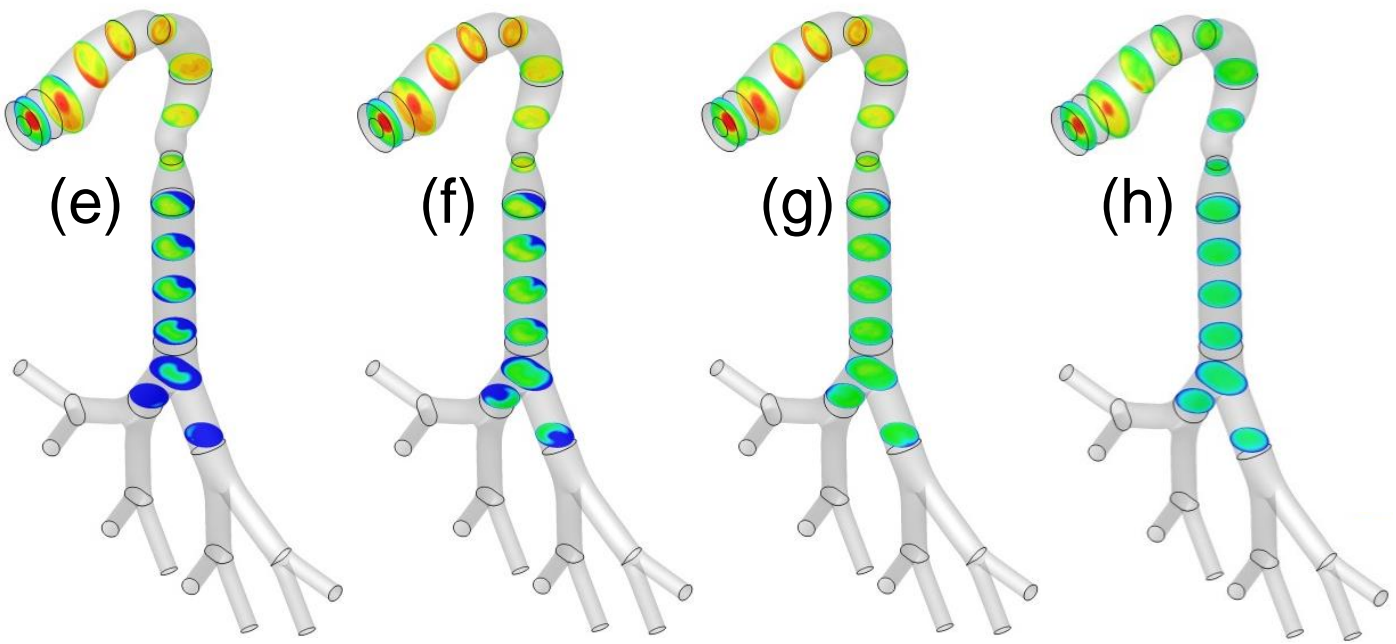

Fig. 13: Contours of non-dimensionalized nicotine vapor mass fraction $\tilde{Y}_{s, v}=Y_{s, v} / Y_{s, v}^{(i n)}$ at different cross sections perpendicular to the centerline of the idealized human upper airway geometry at different times: (a) $\mathrm{t}=0.1 \mathrm{~s}$; (b) $\mathrm{t}=0.2 \mathrm{~s}$; (c) $\mathrm{t}=0.3 \mathrm{~s}$; (d) $\mathrm{t}=0.4 \mathrm{~s}$; (e) $\mathrm{t}=0.5 \mathrm{~s}$; (f) $\mathrm{t}=0.6 \mathrm{~s}$; (g) $\mathrm{t}=1.0 \mathrm{~s} ;$ and $(\mathrm{h}) \mathrm{t}=2.4 \mathrm{~s}$ 


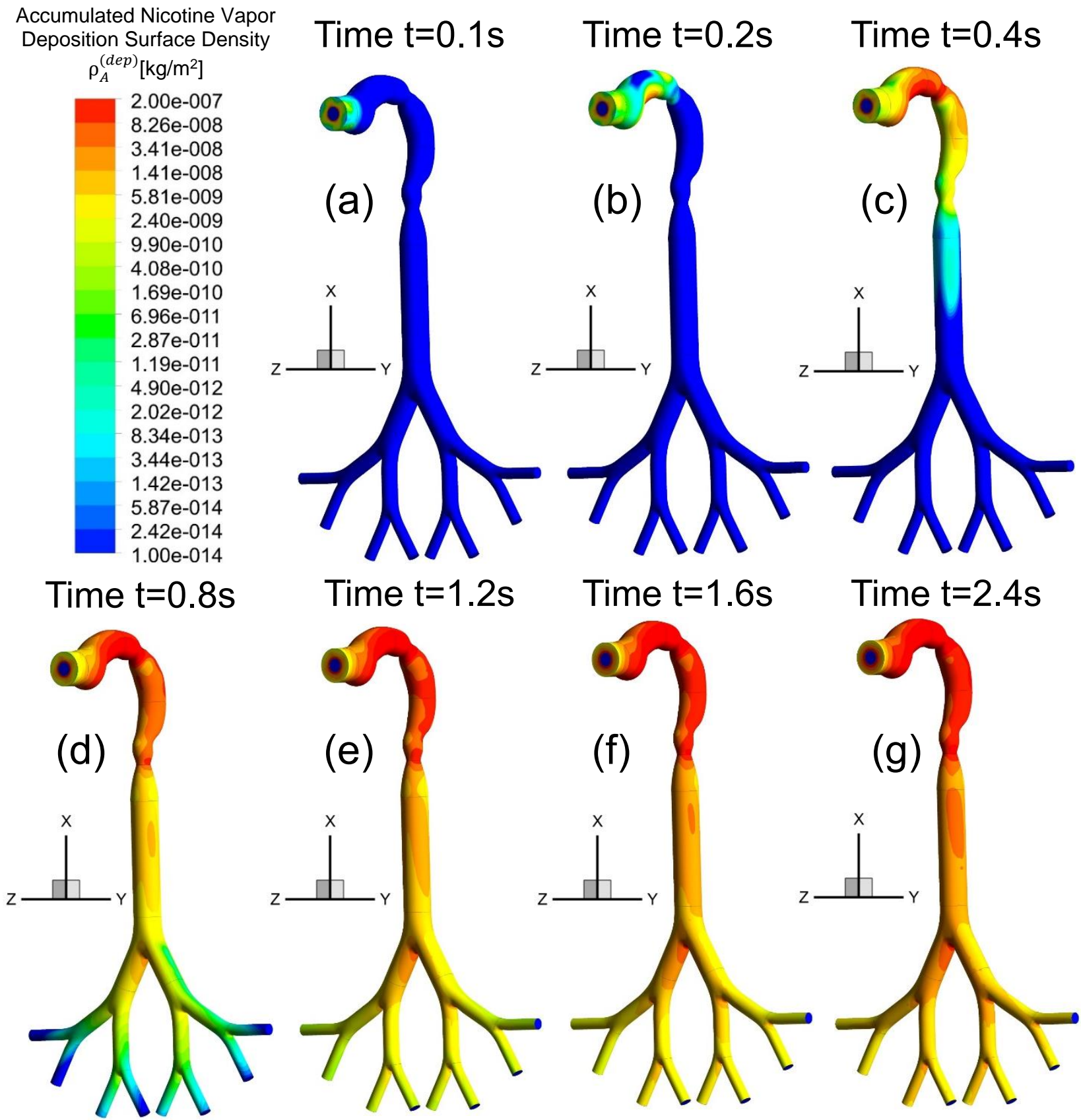

Fig. 14: Contours of surface density $\left[\mathrm{kg} / \mathrm{m}^{2}\right]$ of nicotine-vapor deposition for EC-waveform \#1 at different times: (a) $\mathrm{t}=0.1 \mathrm{~s}$; (b) $\mathrm{t}=0.2 \mathrm{~s}$; (c) $\mathrm{t}=0.4 \mathrm{~s}$; (d) $\mathrm{t}=0.8 \mathrm{~s}$; (e) $\mathrm{t}=1.2 \mathrm{~s}$; (f) $\mathrm{t}=1.6 \mathrm{~s}$; and (g) $\mathrm{t}=2.4 \mathrm{~s}$ 


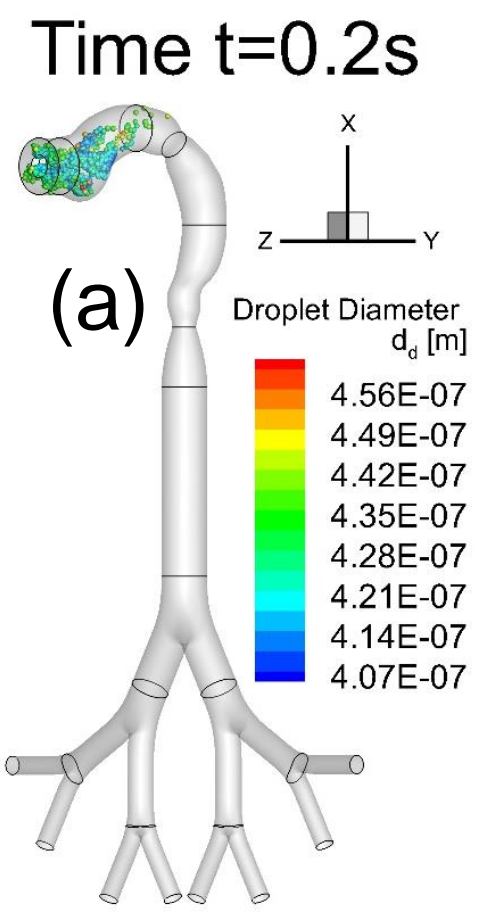

Time $\mathrm{t}=0.8 \mathrm{~s}$

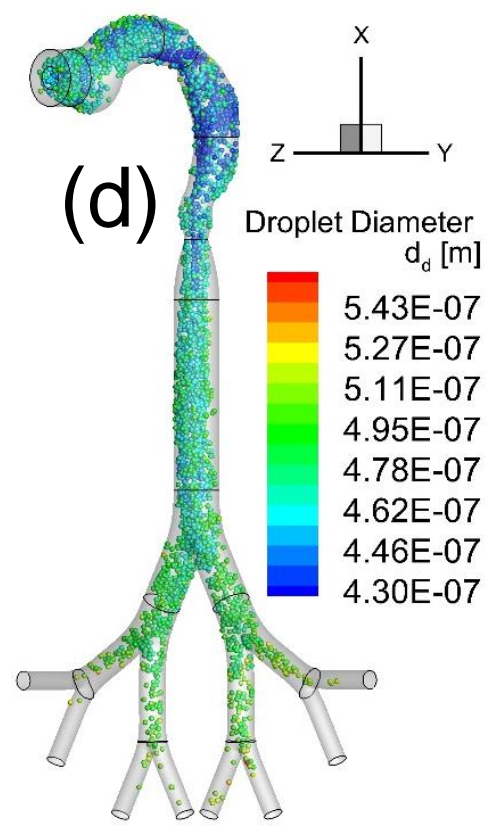

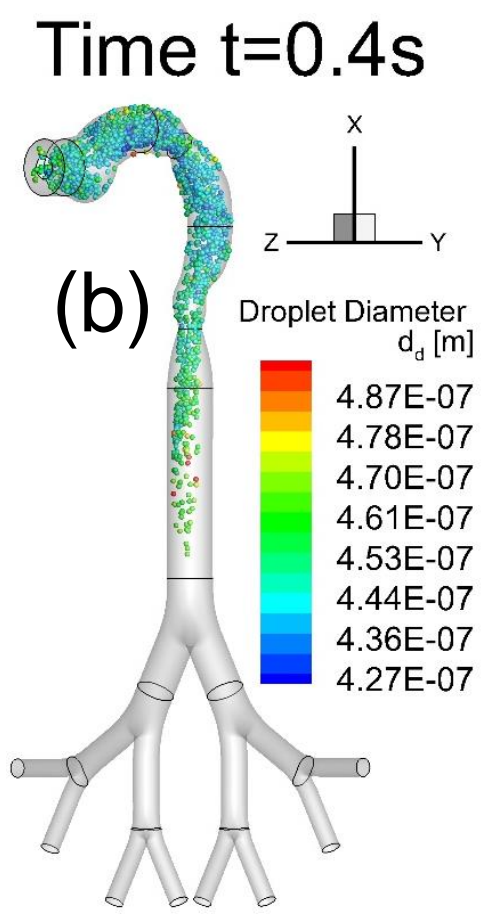

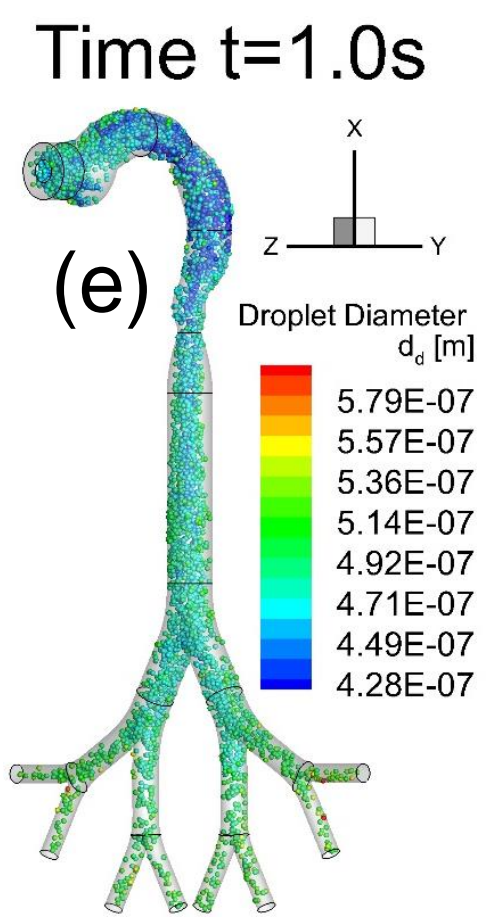

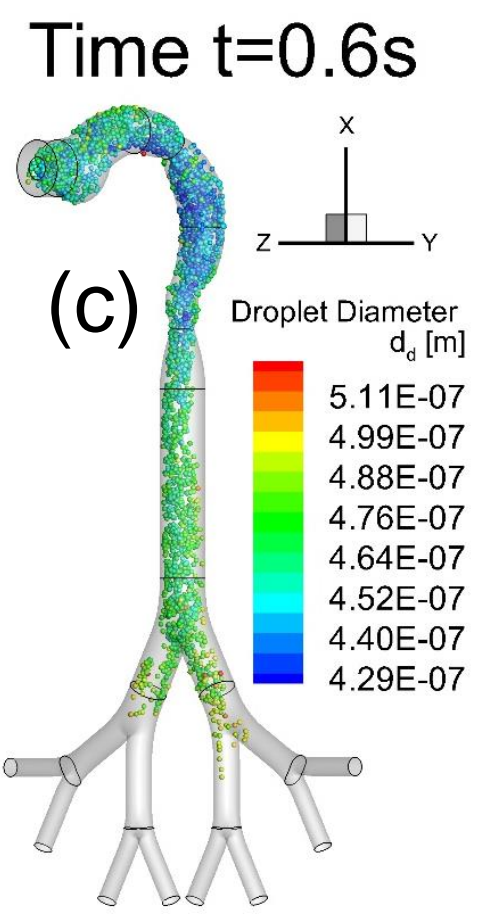
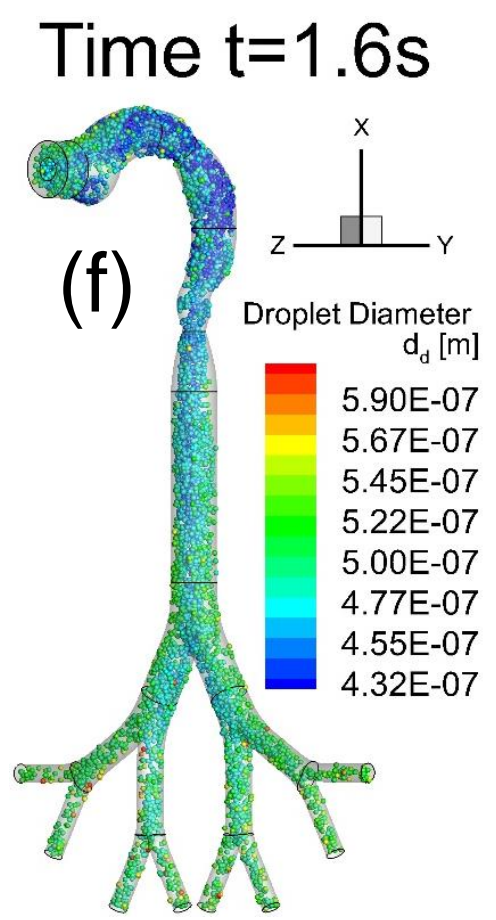

Fig. 15: EC-droplet transport and size change dynamics for waveform \#1 at different times: (a) $\mathrm{t}=0.2 \mathrm{~s}$; (b) $\mathrm{t}=0.4 \mathrm{~s} ;$ (c) $\mathrm{t}=0.6 \mathrm{~s}$; (d) $\mathrm{t}=0.8 \mathrm{~s} ;$ (e) $\mathrm{t}=1.0 \mathrm{~s}$; and (f) $\mathrm{t}=1.6 \mathrm{~s}$ 


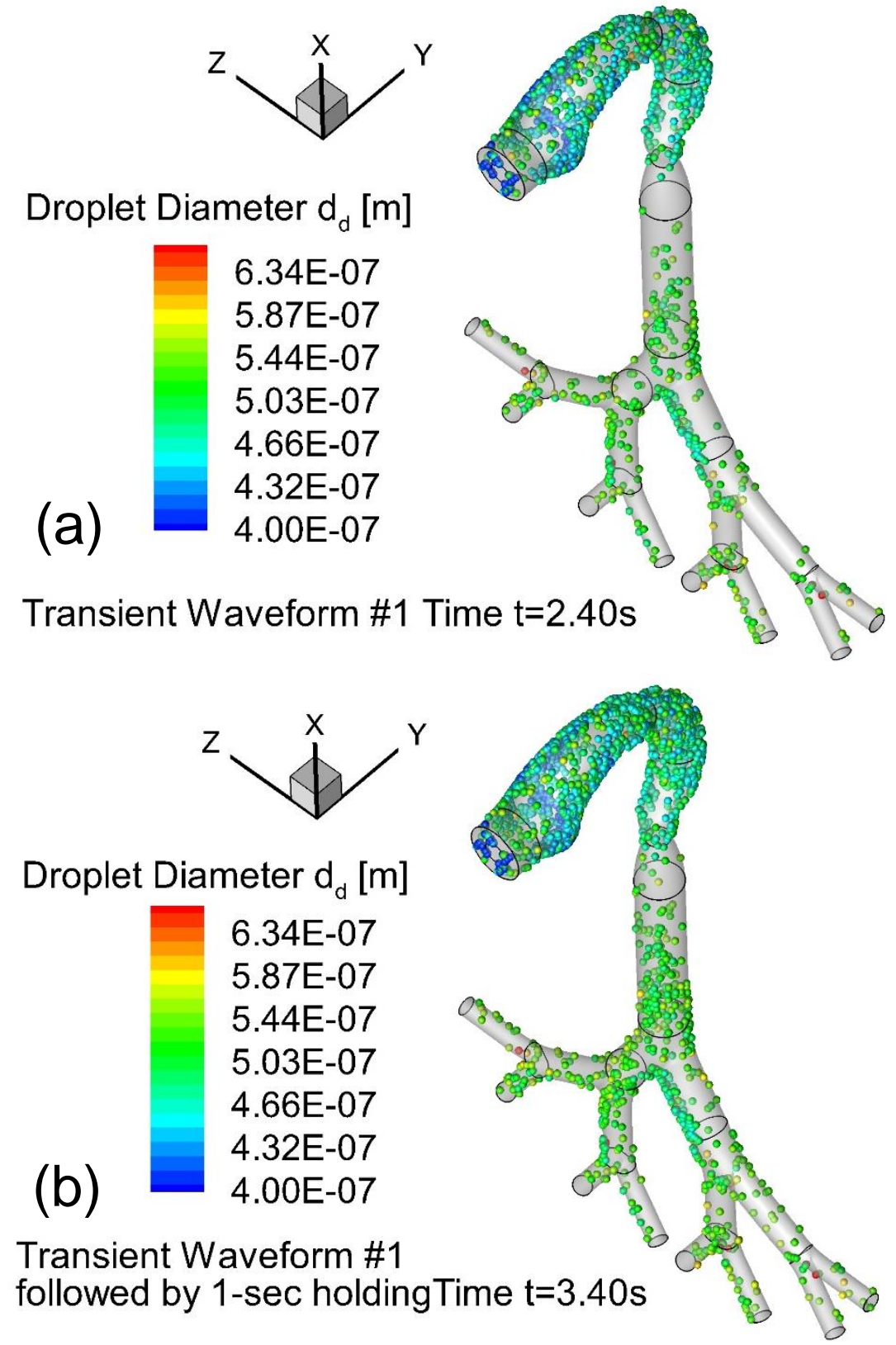

Fig. 16: EC-droplet deposition colored by final diameter: (a) for waveform \#1 at $\mathrm{t}=2.4 \mathrm{~s}$; and (b) for waveform \#1 followed by one-second holding at $\mathrm{t}=3.4 \mathrm{~s}$. 

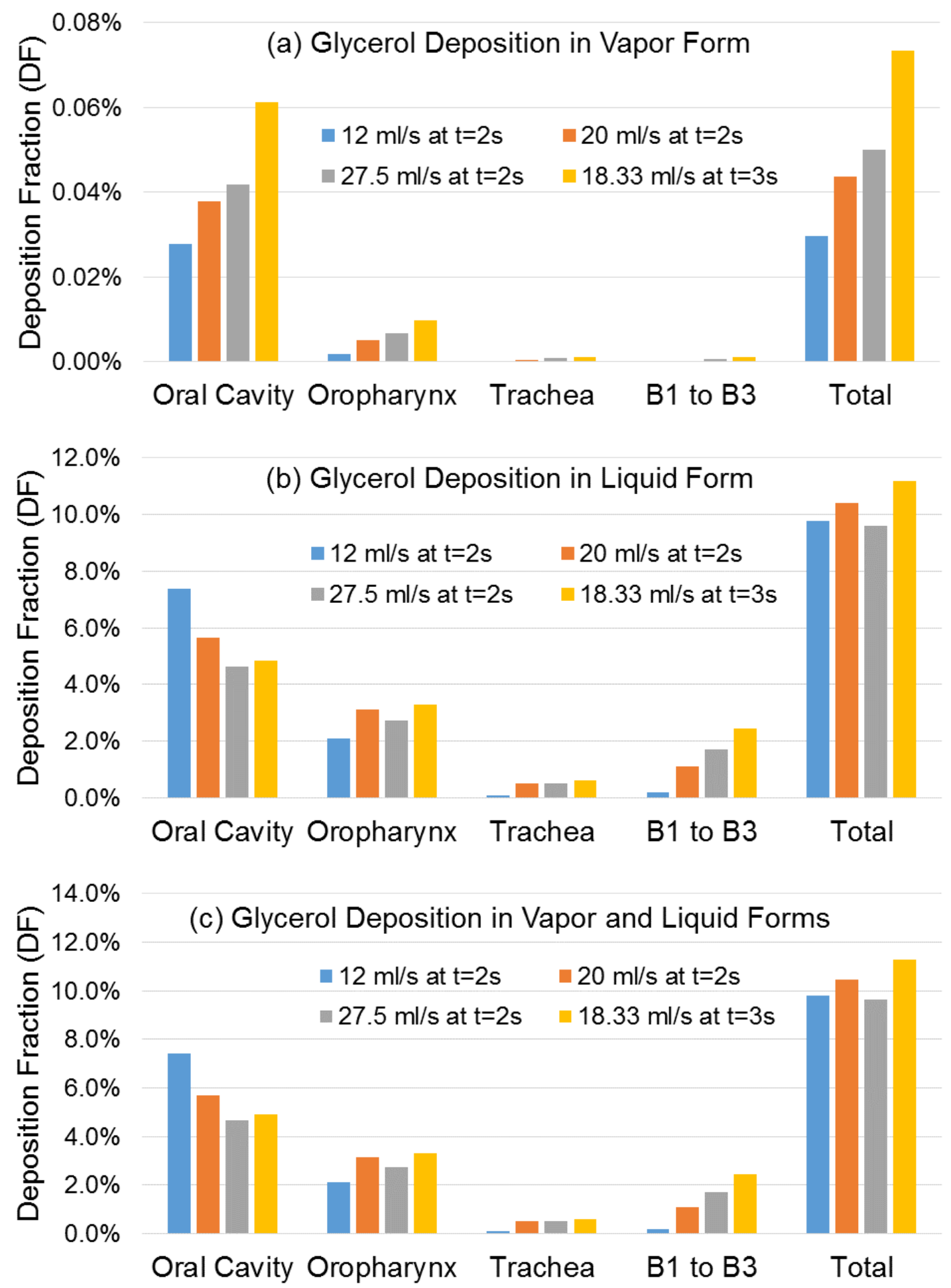

Fig. 17: Regional and total deposition fractions of glycerol considering four different steady puffing scenarios: (a) deposition fractions in vapor form; (b) deposition fractions in liquid form; and (c) deposition fractions in both vapor and liquid forms. 

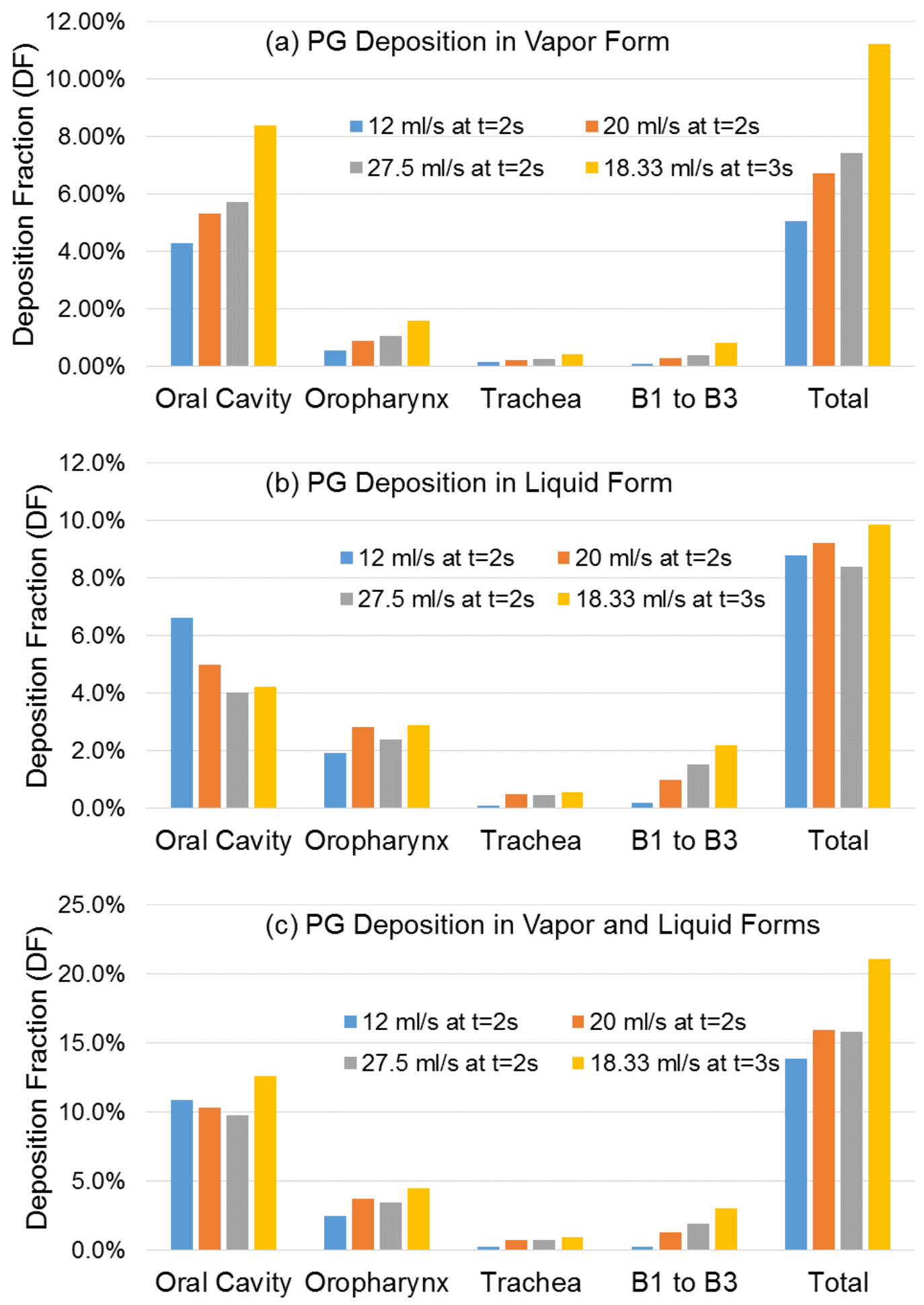

Fig. 18: Regional and total deposition fractions of PG considering four different steady puffing scenarios: (a) deposition fractions in vapor form; (b) deposition fractions in liquid form; and (c) deposition fractions in both vapor and liquid forms. 

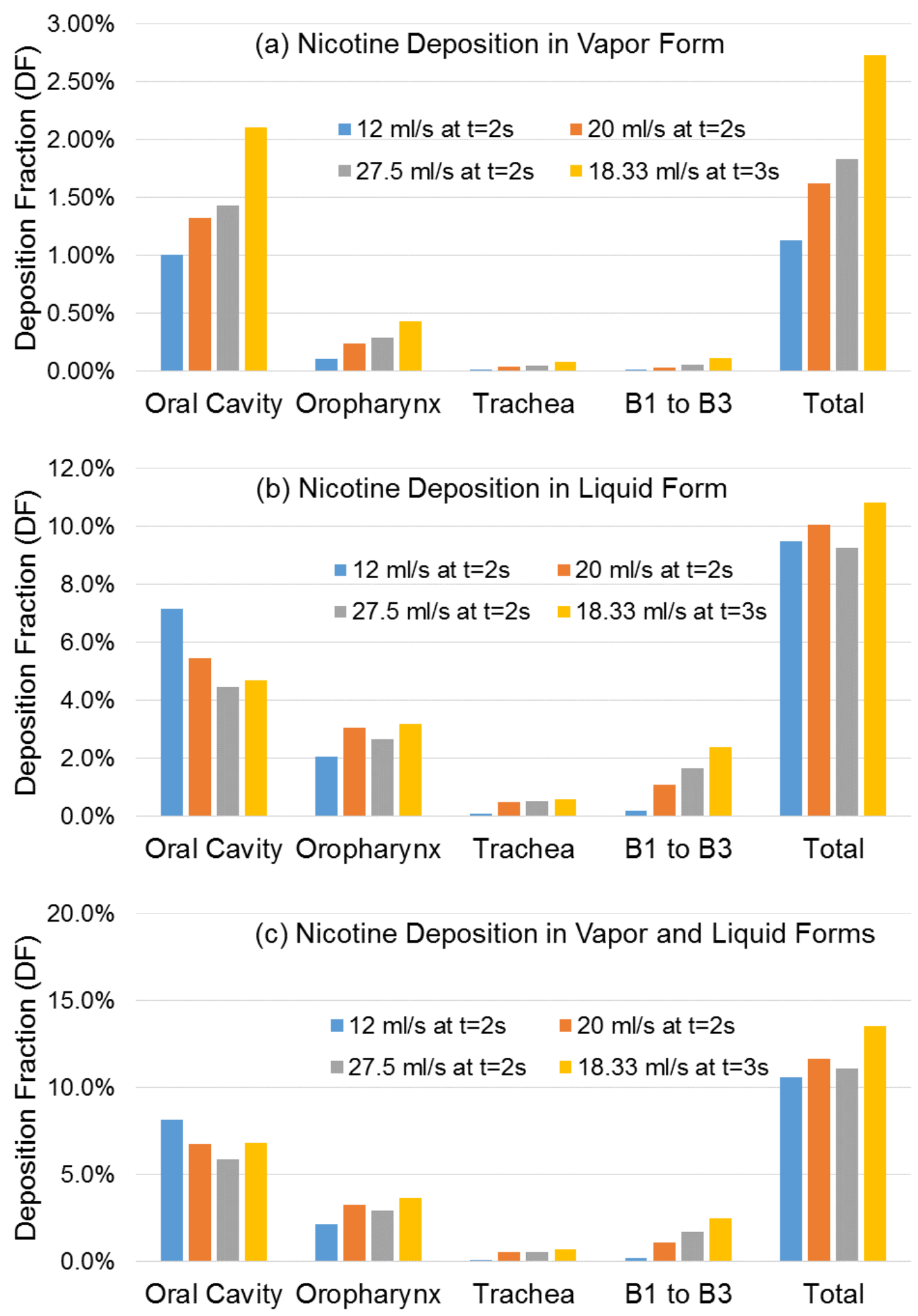

Fig. 19: Regional and total deposition fractions of nicotine considering four different steady puffing scenarios: (a) deposition fractions in vapor form; (b) deposition fractions in liquid form; and (c) deposition fractions in both vapor and liquid forms. 

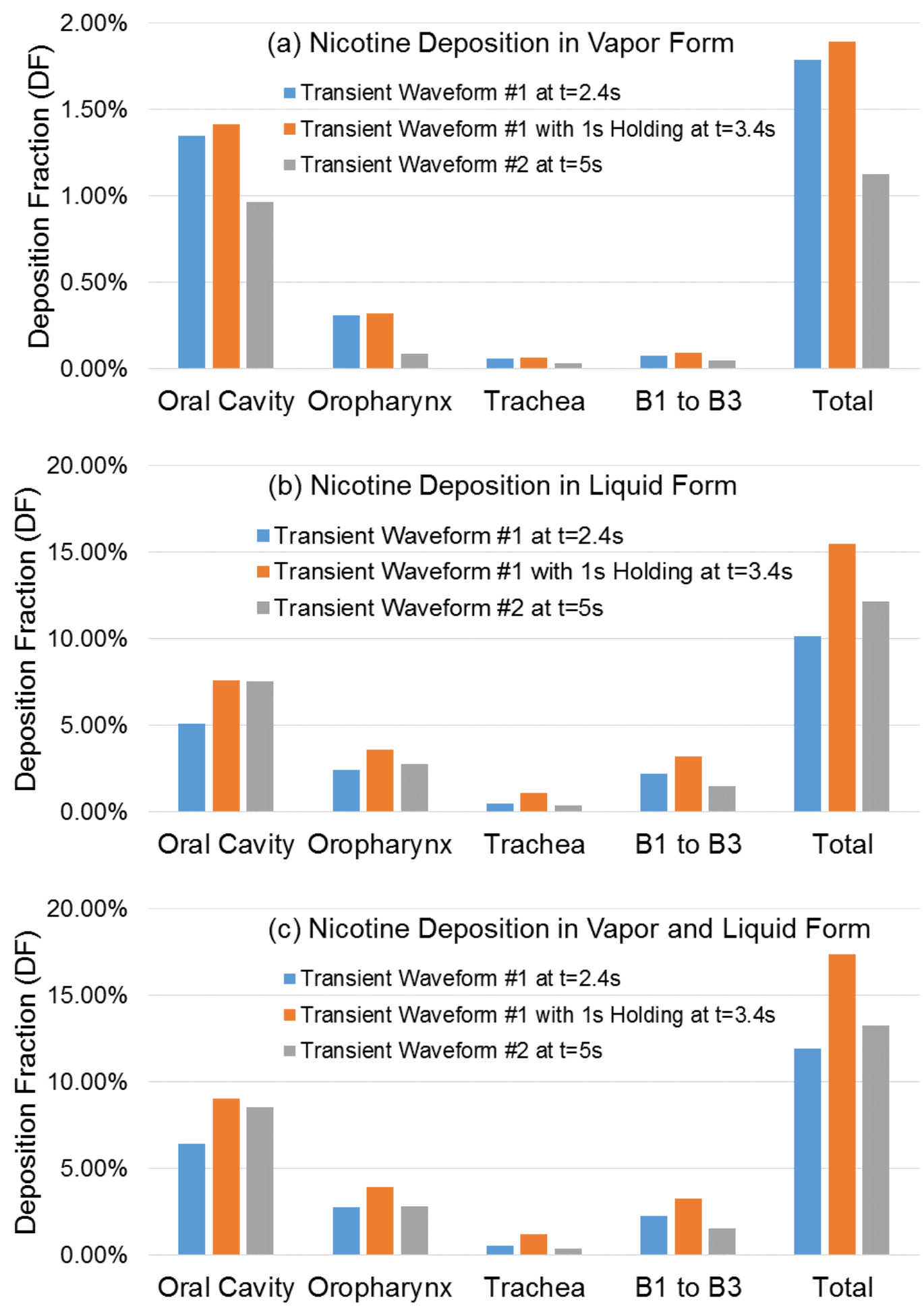

Fig. 20: Regional and total deposition fractions of nicotine for different transient EC-smoke inhalation conditions (i.e., waveform \#1, waveform \#1 with one-second holding, and waveform \#2): (a) deposition fractions in vapor form; (b) deposition fractions in liquid form; and (c) deposition fractions in both vapor and liquid forms. 
(a) Nicotine Deposition in Liquid Form

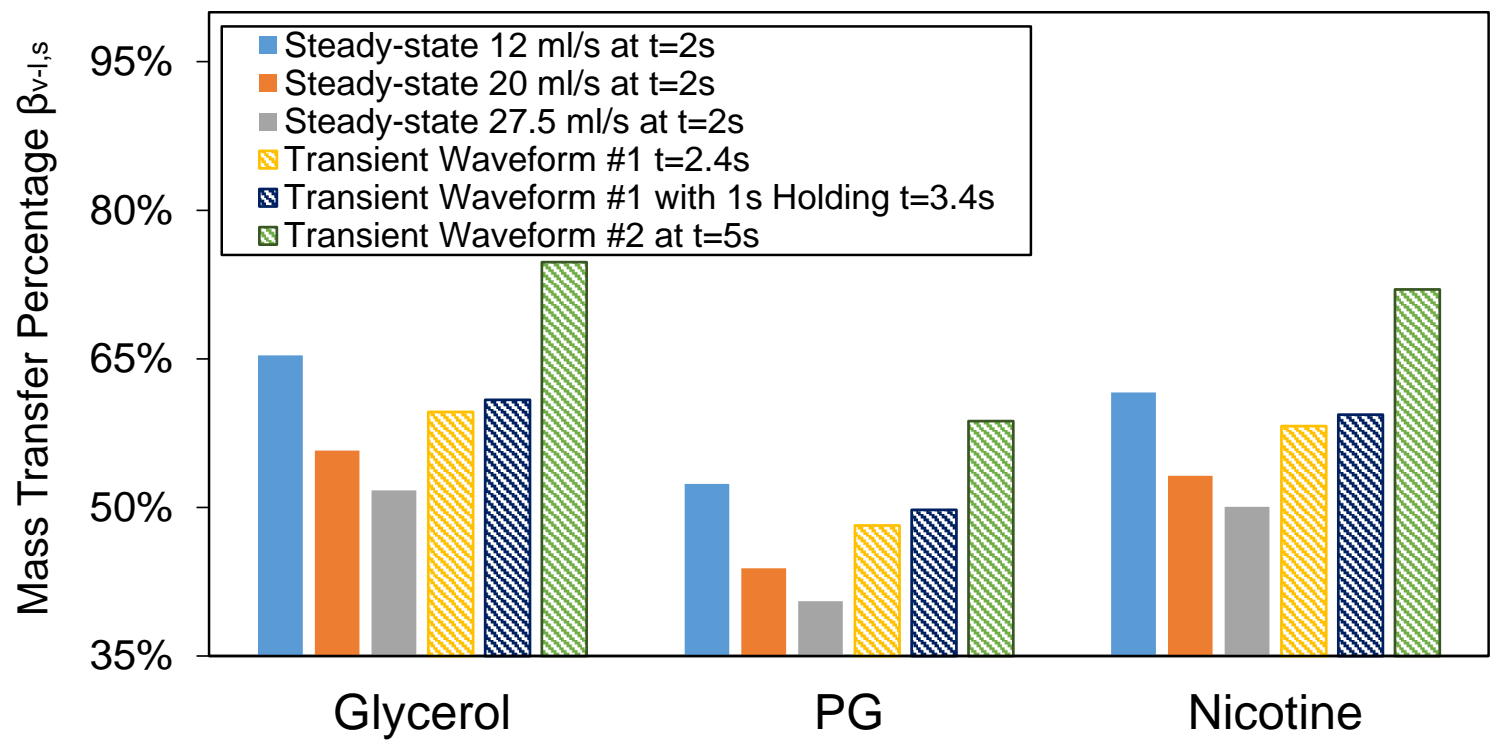

(b) Size Change Dynamics of Suspending Droplets

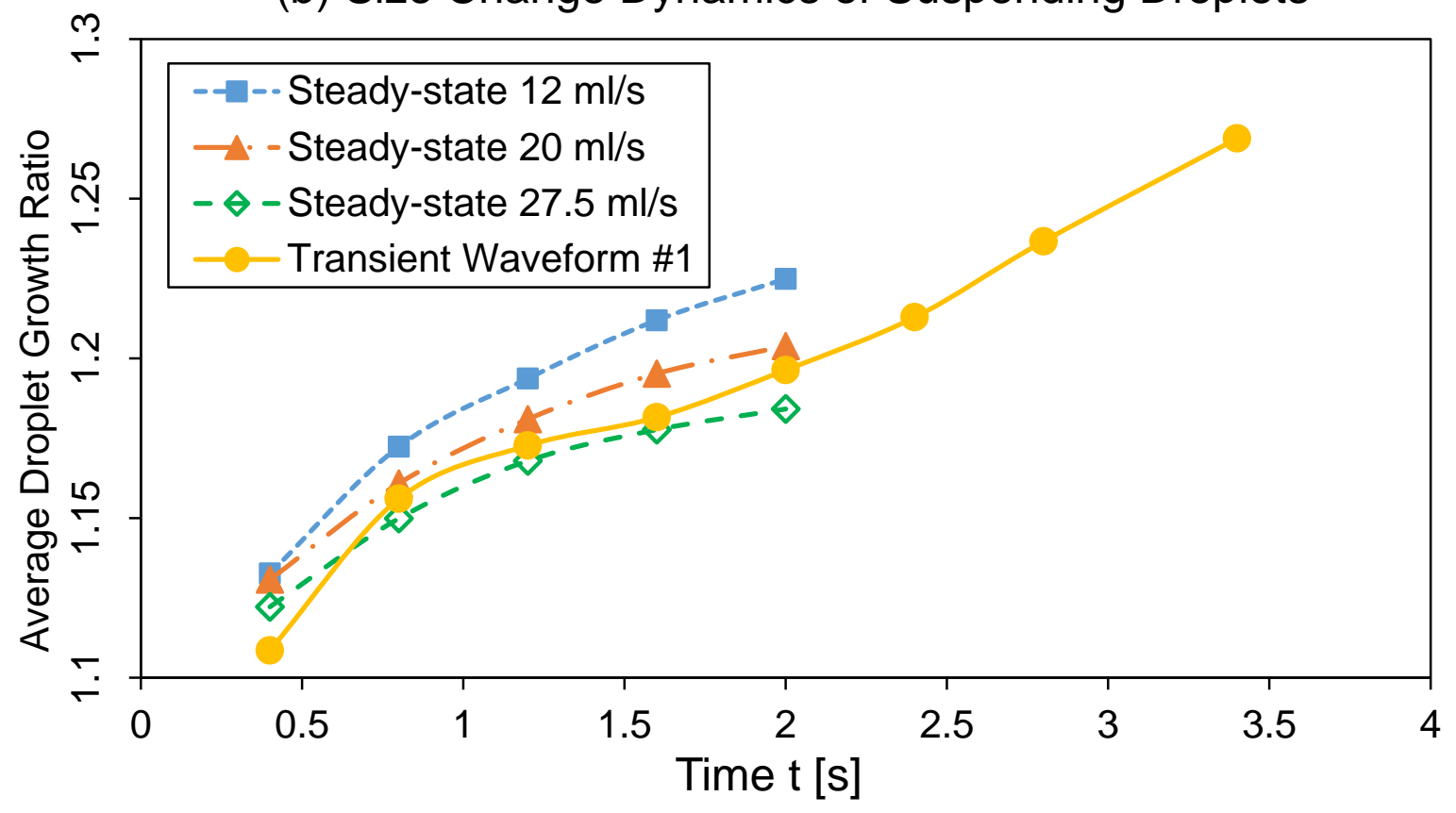

Fig. 21: Comparisons of key aspects of droplet-vapor dynamics for different puffing scenarios: (a) percentage-changes in liquid-vapor mass transfer; and (b) averaged droplet-size growth ratio. 